AN ANALYSIS OF

MARTIAN PHOTOMETRY

AND POLARIMETRY

J. B. POLLACK and C. SAGAN

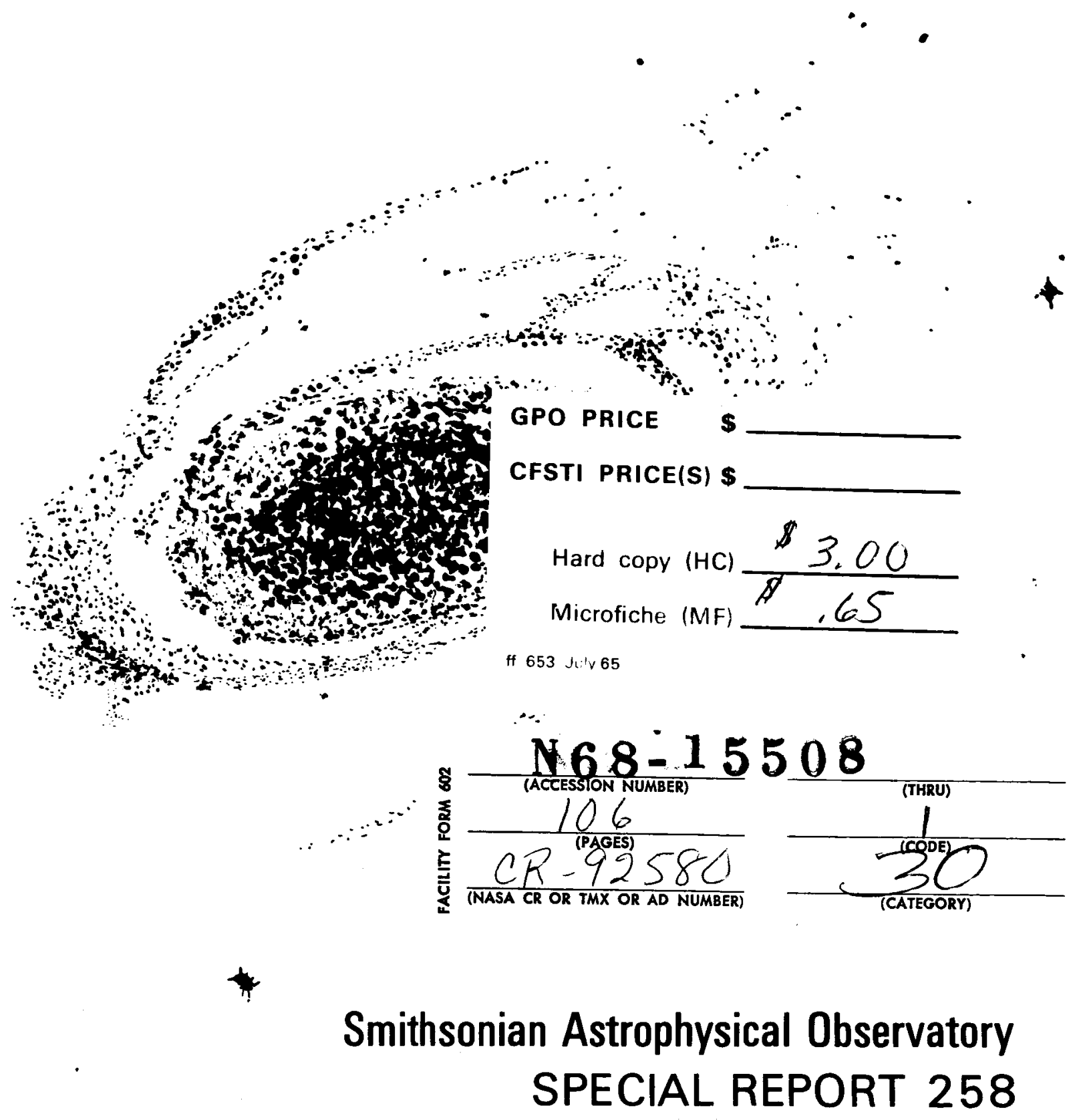


Research in Space Science

SAO Special Report No. 258

\section{AN ANALYSIS OF MARTIAN PHOTOMETRY AND POLARIMETRY}

James B. Pollack and Carl Sagan

November 29, 1967

Smithsonian Institution Astrophysical Obse rvatory Cambridge, Massachusetts 02138 
TABLE OF CONTENTS

Section

$\underline{\text { Page }}$

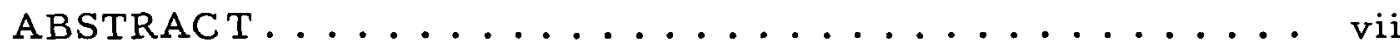

$1 \quad \operatorname{INTRODUCTION} \ldots \ldots \ldots \ldots \ldots \ldots$

2 PHOTOME TRY OF THE BRIGHT AND DARK AREAS ..... 3

2. 1 Visible Wavelength Photometry and Its Implications . . . 3

2.1.1 The photometric data ............. 3

2.1.2 Multiple scattering on the Martian surface .....4 4

2.1.3 Composition of the Martian surface........6 6

2.1.4 Comparison with laboratory data ......... 7

2.1.5 Determinations of particle sizes......... 12

2.1.6 A check on the photometric data......... 18

2.2 Infrared Photometry and Its Implications . . . . . . 20

2.2.1 The "limonite band".............20

2.2. 2 Composition of the Martian surface........ 25

2. 2. 3 Bound-water content of the Martian surface .... 28

2.2.4 The rmal emission and composition ........ 34

3 POLARIME TRY OF THE BRIGHT AND DARK AREAS . . . 39

3. 1 Nature and Reliability of the Data ........... 39

3. 2 Influence of the Martian Atmosphere on the Polarization

Curves..................... 42

3.3 Physics of the Polarization Curves ........... 47

3.4 Implications of the Martian Polarization Curves . . . . 55

3.4.1 Analytic strategy ............... 55

3.4 .2 Determining $\mathrm{n}_{\mathrm{r}}$ and $\mathrm{y} \ldots \ldots \ldots . \ldots . \ldots 56$

3.4.3 Comparison of bright- and dark-area

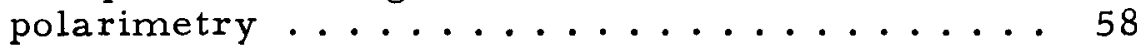

3.4.4 Compositional similarity of bright and dark

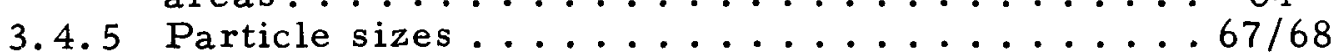

4 ON THE NATURE OF THE "BLUE HAZE" .......... 69

4.1 An Alternative Hypothesis ............... 69

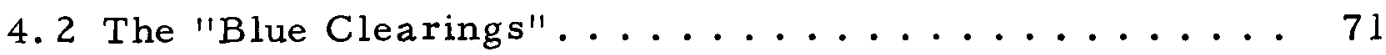




\section{TABLE OF CONTENTS (Cont.)}

Section

Page

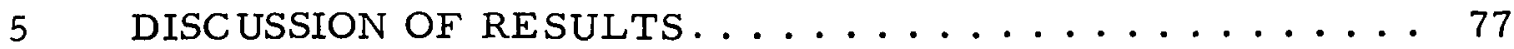

5.1 Summary of Conclusions ............... 77

5.2 Objections to the Model. . . . . . . . . . . 80

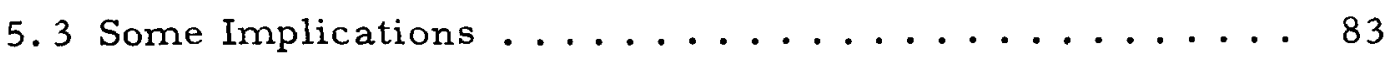

5.4 Recommendations for Future Work .......... 86

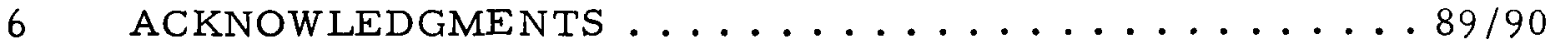

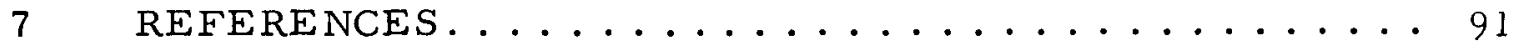

BIOGRAPHICAL NOTES 


\section{LIST OF ILLUSTRATIONS}

Figure

Page

1 Comparison of the observed reflectivity, $K$, of the Martian bright areas (indicated by the squares) with the reflectivity of a laboratory sample of pulverized goethite (indicated by the solid line) . . . . . . . . . . .

2 Comparison of the observed contrast between Martian bright and dark areas (lowest curve) with the laboratory contrast between different mean particle sizes of pul-

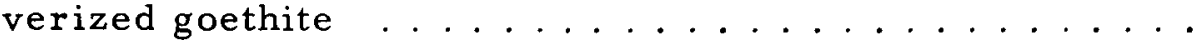

3 Comparison of normalized reflectivity of the integrated Martian disk as a function of wavelength, determined by Younkin (1966) (filled circles), with laboratory data on

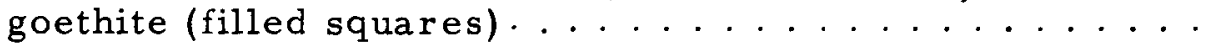

4 Comparison of the geometric albedo of the integrated Martian disk as a function of wavelength, determined by Tull (1966) (filled circles), with laboratory data on

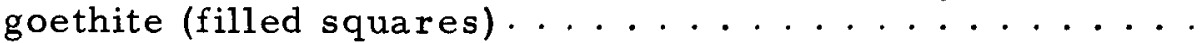

5 Comparison of the normalized reflectivity of the integrated Martian disk as a function of wavelength, determined by Sinton (1966) (open circles and crosses), with laboratory data on goethite (filled squares) ........

Contrast between dark and bright areas of Mars in the interval 7000 and $11,000 \AA$, measured by Younkin (1966) (crosses and open symbols), compared with the contrast among various samples of pulverized goethite......

7 Comparison of infrared observations of Martian bright areas between 2 and $4 \mu$ by Sinton (1967) (filled circles) with theoretical calculations of the spectrum of pulverized goethite at various temperatures (curves connecting

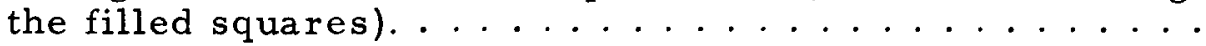

Comparison of infrared observations of Martian dark areas between 2 and $4 \mu$ by Sinton (1967) (filled circles) with theoretical calculations of the spectrum of pulverized goethite at various temperatures (curves connecting

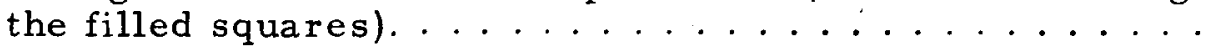

9 Comparison of spectral observations of Mars in the thermal infrared (Sinton and Strong, 1960), given by the dots, with a theoretical blackbody spectrum, given by the dashed line, at a temperature of $273^{\circ} \mathrm{K} \ldots \ldots \ldots$ 


\section{LIST OF ILLUSTRATIONS (Cont.)}

Figure

$\underline{\text { Page }}$

10 Comparison of polarization curves for bright and dark areas of Mars with the corresponding curve for pulverized hematite (curve H). After Dollfus (1957a)......

11 Polarization curves for Martian bright areas near the center of the disk for the four oppositions 1948, 1950, 1952, and 1954. (After Dollfus (1957a)) .........

12 Polarization curves for the Martian bright areas, 1965. (After Dollfus and Focas, 1966.) .............

13 Comparison of the observed (open rectangles, after Dollfus and Focas, 1966) and theoretical values of $\Delta \mathrm{P}(\Phi, \lambda) \mathrm{B}_{\mathrm{S}}(\Phi, \lambda) \ldots \ldots \ldots \ldots \ldots$

14 Schematic representation of fundamental parameters for characterizing polarization curves ..........

15 Wavelength dependence of the polarization curve of a sample of pulverized limonite that matches the Martian observations at a wavelength of $0.63 \mu$. After Dollfus

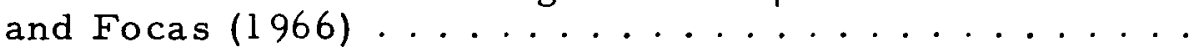

16 A comparison at $0.60 \mu$ of laboratory polarization curves of pulverized limonite for coarse grains (upper curve, $L_{1}$ )

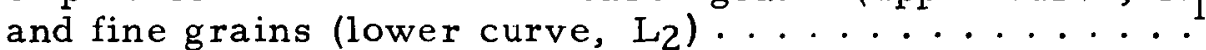

17-20 Polarization curves of selected bright and dark areas of Mars at various times during the 1948, 1950, 1952, and 1954 oppositions for several latitudes. . . . . . . .

21 Correlation of frequency of blue clearing with longitude of the central meridian. (After Slipher, 1962.) ..... 


\section{LIST OF TABLES}

Table

$\underline{\text { Page }}$

1 Reflectivities and contrasts of the bright and dark areas

2 Fallout times from altitudes $h$ in the Martian atmosphere

3 Comparison of bright-area reflectivities derived by de Vaucouleurs (1964) and by Dollfus (1957b), equivalently reduced

4 Parameters for polarization curves uncorrected and corrected for atmospheric polarization

5 Values of the scaling parameters, L, M, and $N$

6 Comparison of derived and observed polarization parameters 


\begin{abstract}
The physical parameters that influence the photometric and polarimetric properties of a solid are enumerated and used to guide a comparison of laboratory measurements with observations of Mars. Both the bright and dark areas of Mars are found to be covered by a fine powder. Furthermore, they appear to have a very similar chemical composition. It is argued that goethite is a major constituent of both regions. The particles on the bright areas are characterized by an average particle radius of $25 \mu$, while those on the dark areas have a mean size of $100 \mu$ outside of the period of seasonal darkening and about $200 \mu$ near the peak of the darkening. The seasonal darkening of the dark areas is the result of a change in the average particle dimension without an accompanying chemical change.
\end{abstract}

The Martian atmosphere has much less of an influence on the photometric and polarimetric observations than was previously supposed. The observed lack of contrast in the blue appears to be largely the result of an intrinsic loss of surface contrast, and not an effect of a hypothetical atmospheric blue haze. 


\section{RÉSUMÉ}

Les paramètres physiques qui influencent les propriétés photométriques et polarimétriques d'un solide sont énumérés et utilisés pour conduire une comparaison entre des mesures de laboratoire et des observations de Mars. On trouve que les régions brillantes et les régions sombres de Mars sont couvertes d'une fine poudre. En outre, elles paraissent avoir des compositions chimiques très semblables. Il est soutenu que la goethite est un constituant majeur des deux régions. Les particules des régions brillantes sont caractérisées par un rayon moyen de $25 \mu$, tandis que celles des régions sombres ont une taille moyenne de $100 \mu$ en dehors de la période d'assombrissement saisonnier, et d'environ $200 \mu$ près du maximum d'assombrissement. L'assombrissement saisonnier des régions sombres est le résultat d'une variation de la dimension moyenne des particules, non accompagné d'un changement chimique.

L'influence de l'atmosphère Martienne sur les observations photométriques et polarimétriques est bien inférieure à ce qui était supposé antérieurement. Le manque de contraste que l'on observe dans le bleu, parait être principalement une conséquence de la perte de contraste de surface, et non pas un effet d'un hypothétique halo atmosphérique bleu. 
HOHCITFKT

Физические параметры, имеюие влияние на фотометрические и полариметрические свойства твердого тела, перечисляктся и vпотребляются как руковолство для сравнения межпт лабораторными измерениями т наблюдениями Марса. Обе, светлая и темная пбласти Manca бнли найтены покрытыми мелкой пнлью. Fпоме того. Снт произвопили впечатление имеюाих схожий химическй состав. Обсужлается воп口ос, является ли гетит главно: составной частни обеих областей. Частиты в светлых областяж характепивvются паличсом срелней частины в $25 \mu$ тогпа как частицы в темных областях имеют средний размер в $100 \mu$ за препелами пепитпа сезонного затемнения и в $200 \mu$ вблизи максимума затемнения. Сезонное затемнение темных областей является пезультатом измепения размера частит тооисхотяшего без химического изменения.

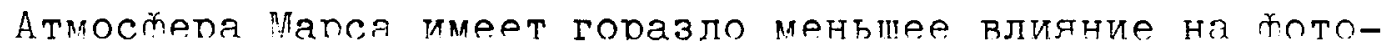
метпические и полапиметьические наблюления чем ппеппопагалось паньіе. Наблюпаемый непостаток контпаста в синей области кажется пропсхопящим, в основном, как певультат пейтвттельной Потепи пORепхностного контраста а не является ревультатом Гипо-

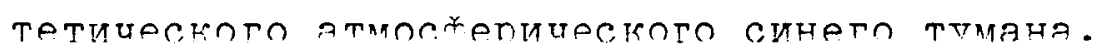




\title{
AN ANALYSIS OF MARTIAN PHOTOMETRY
}

\author{
AND POLARIME'TRY
}

James B. Pollack and Carl Sagan

\section{INTRODUCTION}

Clues regarding the nature of the bright and dark areas of Mars can be found in the photometric and polarimetric properties of these regions. In the past it has been customary to compare the observations of Mars with laboratory data to derive properties of the planetary surface (e. g. , Dollfus, 1957a; Sharonov, 1961; Sagan, Phaneuf, and Ihnat, 1965). A principal result has been the suspicion that pulverized ferric oxide polyhydrates, such as limonite, are important constituents of the Martian surface. In the present paper we seek to refine the laboratory comparisons, to approach the problem from a somewhat more fundamental physical viewpoint, and to derive limits on the acceptable ranges of the several parameters that cha racterize the surface, such as particle size, degree of compaction, and the real and imaginary parts of the index of refraction.

Closely tied to any analysis of Martian photometric properties is the question of the "blue haze," the progressive loss of surface contrast between bright and dark areas of Mars as shorter visible and near-ultraviolet wavelengths are employed. Most observers have concluded, as the description of the phenomenon itself indicates, that an absorbing and/or scattering atmospheric laye $\mathbf{r}$ is responsible (see, e.g. Sagan and Kellogg, 1963). If this were the case, then the layer would make significant contributions to the light seen in short-wavelength photometric observations intended as studies of the planetary surface. However, several lines of evidence-e.g., the enhanced

This work was supported in part by grant NGR-09-015-023 from the National Aeronautics and Space Administration. This paper is dedicated to the memory of $V$. V. Sharonov. 
contrast in the blue and violet of the polar caps, and ultraviolet rocket spectroscopy of Mars - strongly counterindicate the notion of a blue haze, and suggest that the phenomenon is due to an intrinsic loss of surface contrast at short visible wavelengths. We justify these ideas, and discuss such associated problems as the blue clearings, below. For the moment we adopt the hypothesis that the photometric measurements of Mars at wavelengths, $\lambda$, longward of $4000 \AA$ pertain primarily to the surface. At shorter wavelengths, Rayleigh scattering by a $10-\mathrm{mb}$ atmosphere will become increasingly important. The contribution of the gaseous and particulate components of the atmosphere to the polarimetric results will be discussed separately.

The photometric, polarimetric, and blue-haze observations, and their interpretations, will now be treated in that order. The photometric observations will be discussed approximately in order of increasing wavelength. 


\section{PHOTOMETRY OF THE BRIGHT AND DARK AREAS}

\section{1 Visible Wavelength Photometry and Its Implications}

\subsubsection{The photometric data}

The parameter $\mathrm{K}$ is the reflectivity for angle of incidence = angle of reflection $\simeq 0^{\circ}$ (but not so near to $0^{\circ}$ that one runs into the opposition effect); i. e., it is the ratio of the observed surface brightness due to backscattering by an area oriented perpendicular to the incident sunlight to that due to a backscattering by a perfectly diffusing white screen (i. e., a nonabsorbing Lambert scatterer) with similar orientation. Values of $\mathrm{K}$ for the Martian bright areas are contained in a compilation by Dollfus and Focas (1966, their Figure 17). Within the scatte $r$ in the data, the $K-\lambda$ observations a re fit adequately by a straight line. As discussed below, these values of $K$ can be checked against those derived from well-established broadband photoelectric photometry of the whole planet, and are found to be in good agreement. Table 1 summarizes these observations as well as the value of the contrast, $\Gamma$, between bright and dark areas obtained in an earlier work of Dollfus (1957b); $\Gamma$ is defined as the difference between $\mathrm{K}$ for bright and dark areas divided by its bright-area value. The dark areas measured are prominent features of both Northern and Southern Hemispheres; measurements were made during the 1950 and, particularly, the 1952 oppositions, but not at the peak of the seasonal darkening. The value of $K$ for the dark areas can be inferred from $\Gamma$ and from $K$ for the bright areas. Values of $\Gamma$ are probably good to $10 \%$ (Dollfus, private communication, 1966), while the values of $\mathrm{K}$ are, judging from the scatter in the data points, good to no better than $20 \%$.

We see from Table 1 that both bright and dark areas exhibit a marked rise in reflectivity between $4500 \AA$ and $6500 \AA$, although the bright-area slope is the steeper. The table helps to clarify the confusing statements that have appeared in the literature on the color of the dark areas. The dark areas are red, although not as red as the bright areas. This conforms to the visual 
impression of Kuiper (1957), who described the dark areas as having the appearance of shadows cast on the bright areas. Much of the reports of dark-area coloration are attributable to psychophysiological effects: A relatively neutral-colored area adjacent to a brightly colored area takes on colors complementary to those of the bright area (Schmidt, 1959; Hurwich and Jameison, 1965). Complementary to the oranges and reds of the bright areas are greens and blues. If the albedo of the dark areas varies during the seasonal darkening, the associated psychophysiological colors should vary as well. This also has been reported.

Table 1. Reflectivities and contrasts of the bright and dark areas

\begin{tabular}{|c|c|c|c|}
\hline$\lambda$ & $\mathrm{K}$, bright area & $\mathrm{K}$, dark area & $\Gamma$ \\
\hline $4000 \AA$ & - & - & 0.04 \\
4500 & 0.071 & 0.065 & 0.08 \\
5000 & 0.120 & 0.098 & 0.18 \\
5500 & 0.164 & 0.120 & 0.27 \\
6000 & 0.210 & 0.139 & 0.34 \\
6500 & 0.250 & 0.150 & 0.40 \\
\hline
\end{tabular}

\section{1.2 Multiple scattering on the Martian surface}

We now proceed to some of the qualitative implications of Table 1 . The sharp rise in reflectivity between 4500 and $6500 \AA$ indicates multiple scattering of sunlight by the Martian surface. Spectrophotometry of solid samples of hydrated ferric oxides shows a low and nearly constant reflectivity in this wavelength region, while powders of the same materials exhibit higher reflectivities with more marked wavelength dependence (Sagan et al., 1965). This behavior can be understood in general terms, independently of the precise composition of the Martian surface.

For an optically thick slab, reflected light is produced at the air-solid interface and depends on $\left|n_{c}-1\right|$, where $n_{c}$ is the complex index of refraction. For all nonmetallic materials, in the visible and near infrared, 
$n_{i} \ll n_{r}$, where $n_{i}$ and $n_{r}$ are, respectively, the imaginary and real parts of the complex refractive index, so that $\left|n_{c}-1\right| \equiv\left|n_{r}-i n_{i}-1\right| \simeq\left|n_{r}-1\right|$. This result is readily derived. Let $k$ be the absorption cross section per unit volume; light passing through a material of thickness, $x$, is then attenuated by $\exp (-k x)$. From classical electromagnetic theory, $n_{i}=k \lambda / 4 \pi$. Most materials at these wavelengths, even the relatively opaque iron oxides, become transparent as $x \rightarrow \lambda$; for $x \simeq \lambda$, $k x \leq 1$, and $n_{i} \ll 1$. If this were not true for iron oxides, even small particles would be opaque, and the reflectivity would be unaffected by changes in particle size. Instead there is a marked increase in reflectivity with decreasing particle size between $0.5 \mathrm{~mm}$ at $50 \mu$ (Sagan et al. , 1965; Hovis, 1965). Similar results apply to other materials (cf. Gray, 1963).

Since particle sizes in laboratory experiments are usually determined by sieving, it is possible that small grains are still present among the "large" particles because of cohesion. This is unlikely to be a dominant effect, because of the systematic increase of reflectivity with decreasing particle size. In any case there is no reason to expect that such cohesion will be unimportant on Mars if it is important on Earth, and all subsequent discussion of particle size can also be understood as including cohering smaller grains.

By the Kramers-Kronig relationship, $n_{r}$ varies with wavelength if and only if $n_{i}$ becomes comparable to $n_{r}$. Since $n_{i}$ is small for a solid slab in the visible and near infrared, $\mathrm{n}_{\mathbf{r}}$ and the reflectivity will vary only slightly with wavelength through the visible (cf. Pollack and Sagan, 1965a; Irvine and Pollack, 1967).

On the other hand, if the material is powdered (or very porous), multiple scattering can occur. When a photon enters a small, optically thin particle, there is a substantial probability of its emerging from the sides or rear of the particle, suffering subsequent reflections, and eventually escaping from the solid. If a is a characteristic particle size, exp(-ka) gives approximately the transmissivity of an individual particle. If $\mathrm{ka} \ll 1$, multiple scattering occurs and leads to high reflectivities as viewed from outside the scattering medium. Moreover, since $n_{i}$ and $k$ may vary rapidly through the visible, 
the reflectivity resulting from multiple scattering may have strong wavelength dependences. Iron oxides that have flat spectra when in solid slabs characteristically show a marked $\lambda$-dependence when pulverized (Sagan et al., 1965).

Observations of yellow clouds having closely similar photometric properties to those of the bright areas indicate that the materials of the bright areas exist as a powder, not as a porous rock. The low value of the thermal inertias of the bright areas (Sinton and Strong, 1960; Leovy, 1966) supports this conclusion.

\section{1.3 Composition of the Martian surface}

We now consider the implications of Table 1 for composition. At $4500 \AA$, the reflectivity becomes small and comparable to that of optically thick solid samples, indicating that the individual particles of both bright and dark areas are becoming opaque and single scattering is beginning to dominate. When we require the particles to be small enough to be raised off the surface and carried appreciable distances by winds (see quantitative discussion below), we find that the material of the bright areas must be strongly absorbing in the visible. Small particles (say, conservatively, a $1 \mathrm{~mm}$ ) of most geochemically abundant materials remain transparent to the middle ultraviolet. For example, a quartz particle, $a=1 \mathrm{~mm}$, does not become optically thick until about $1200 \AA$; the corresponding wavelength for $\mathrm{Al}_{2} \mathrm{O}_{3}$ is $1400 \AA$ (Gray, 1963). Small particles of iron oxides, on the other hand, can become quite opaque at $4500 \AA$, and rapidly become semitransparent with increasing wavelength, again in agreement with the observations of Mars. Iron compounds are virtually the only geochemically abundant materials with such strong blue absorption; most electronic transitions in solids occur at shorter wavelengths. Quantitative comparisons will be carried out below. Note that if iron oxides are responsible for the opacity at visible frequencies of the Martian dust, we cannot yet conclude they are a major constituent - precisely because such other common materials as quartz are transparent in the visible and make negligible contribution to $k$. The visible opacity of small grains of terrestrial sand and dust is chiefly due to iron oxides present as impurities. However, we can approach this important question by determining the particle sizes 
for iron oxides required to match the photometric observations, and then comparing our result with independent estimates of the actual particle size. This comparison also is performed below.

Perhaps the most provocative implication of Table 1 is a close compositional similarity between bright and dark areas. Both exhibit a very low reflectivity at $4500 \AA$ and a rapidly increasing reflectivity between 4500 and $6500 \AA$, data that we have taken to indicate the presence of iron oxides. Even more significant is the low value of the contrast between bright and dark areas at 4000 to $4500 \AA$. The low values of the reflectivities at these wavelengths - comparable to the reflectivities of solid samples - together with the near constancy of reflectivity between 3000 and $4500 \AA$ (Evans, 1965) imply that the reflectivity of both bright and dark areas is being controlled by $\mathrm{n}_{\mathrm{r}}$; the reflected light a rises principally from single-scattering events at the air-particle interface. The low values of $\Gamma$ then imply a near identity between $n_{r}$ for the bright and for the dark areas, provided that there is no atmospheric blue haze. The increase in contrast toward longer wavelengths can then be understood in terms of particle size. Toward longer visible wavelengths the reflectivity increases, indicating a transition to semitransparency. The fraction of incident light penetrating the particle, $\simeq \exp (-k a)$, begins to control the reflectivity. Thus, if the dark areas have larger particles (or more of the absorbing material) and so larger optical depths, ka, they will display a lower reflectivity, as observed, while maintaining the same composition.

The foregoing discussion has provided two guiding hypotheses: (1) the bright areas are composed, at least in part, of pulverized iron oxides; and (2) the composition of the dark areas is similar to that of the bright areas, but the mean particle size (or the iron oxide abundance) is larger.

\subsubsection{Comparison with laboratory data}

We now relate Martian photometry to laboratory measurements of various terrestrial samples. A typical laboratory measurement consists of a determination with an integrating sphere of the light reflected in all directions 
when the angle of incidence $\simeq 0^{\circ}$. By contrast, Dollfus' observations are for small angles of both incidence and reflection. We relate the two quantities through the observed limb darkening of the Martian surface. The laboratory reflectivity, $R$, is related to the specific intensity, $I$, of the reflected light, and to the incident flux, $F_{i}$, by

$$
R=\int_{+} I(\mu) \mu d \Omega / F_{i},
$$

where $\arccos \mu$ is the angle of reflection and $d \Omega$ is an element of solid angle. Integration is carried out ove $r$ the forward hemisphere. In an analysis of the infrared limb darkening of the clouds of Venus, we have found that a convenient representation of the limb darkening is

$$
I(\mu)=I(\mu=1) \mu^{a},
$$

where $a$ is a constant (Pollack and Sagan, 1965b). This representation was found to give a good fit to exact limb-darkening laws, derived unde $r$ a variety of assumptions, until very close to the limb. Combining equations (1) and (2), we find

$$
R=\frac{\pi I(\mu=1)}{F_{i}} \quad \frac{2}{2+a}
$$

The reflectivity found by Dollfus (1957b) is

$$
K \equiv \frac{I(\mu=1)}{I^{\prime}(\mu=1)}
$$

where $I^{\prime}(\mu=1)$ is the specific intensity of a perfect diffuse reflector oriented at zero angle of incidence and reflection; it is related to the incident flux by $F_{i}=\pi I^{\prime}(\mu=1)$. Combining this result with equations (3) and (4), we obtain the desired relationship between $\mathrm{R}$ and $\mathrm{K}$ :

$$
R=u K \equiv \frac{2}{2+a} K
$$


Dollfus (1957c) has obtained the $6200-\AA$ brightness variation of the bright areas in equatorial scans for phase angles $10^{\circ}, 20^{\circ}$, and $30^{\circ}$. The parameter a is defined, through equation (2), for zero angle of incidence and varying angles of reflection. For a given phase angle there is only one point on the surface where the sun is directly overhead. Thus, three points can be obtained from these limb-darkening measurements. For each phase angle the brightness peaks at zero angle of incidence. Accordingly, we extract $\mathrm{K}$ from Dollfus' data for angles of reflection of $10^{\circ}, 20^{\circ}$, and $30^{\circ}$, deriving $a \simeq 0.90$ and $u \simeq 0.69$ at $6300 \AA$. Owing to the small differences involved at the three phase angles, and the small number of points, $a$ is uncertain to about $25 \%$; the corresponding uncertainty in $\mathrm{u}$ is about $10 \%$.

The surface limb darkening is insensitive to wavelength, as judged from a comparison of results at 6300 and $5000 \AA$ (Dollfus, 1957c). The absolute reflectivities at these two wavelengths differ by more than a factor of 2; yet at phase angle $29^{\circ}$ the relative values of the brightness, normalized to the peak values, as a function of angular distance across the disk are identical at the two wavelengths within the error of measurement.

We are now in a position to compare the Mars photometry with laboratory reflectivities of pulverized iron oxides obtained by Hovis (1965). The re are several mineralogically distinct, common terrestrial oxides of iron. Magnetite, $\mathrm{Fe}_{3} \mathrm{O}_{4}$, is a mixture of the +2 and +3 oxidation states of iron. Below the Curie point it is ferromagnetic. The other varieties discussed here are not ferromagnetic. In hematite, $\mathrm{Fe}_{2} \mathrm{O}_{3}$, only the +3 oxidation state is present. The name comes from its occasional blood-red appearance. Limonite is usually written as $\mathrm{FeO}(\mathrm{OH}) \cdot \mathrm{n}^{\prime} \mathrm{H}_{2} \mathrm{O}$ or as $\mathrm{Fe}_{2} \mathrm{O}_{3} \cdot \mathrm{n} \mathrm{H}_{2} \mathrm{O}$, where $\mathrm{n}^{\prime}$ and $\mathrm{n} \geq 0$ and a re not necessarily integers. The name alludes to its lemon-yellow color. It is not well defined mineralogically; it characteristically contains hematite, clays, and manganese oxides, and may vary in important respects from sample to sample. In major part, however, limonite is a mixture of goethite and lepidocrocite, with lepidocrocite very much the minor constituent. Lepidocrocite (Greek, "scaly wool") is $\mathrm{FeO}(\mathrm{OH})$ according to X-ray crystallographic studies; goethite (named after the German poet) is often written as $\mathrm{HFeO}_{2}$, but the crystallographic data are apparently still ambiguous (R. Siever, 
private communication, 1967). Goethite samples clearly show the 3- $\mu$ waterof-hydration infrared-absorption band (Hovis, 1965), and thermogravimetric analysis clearly drives off bound water (Sagan et al., 1965). In both cases the ratio of bound-water abundance to ferric oxide abundance is about unity. In the following discussion, therefore, we will mean by goethite those molecules of hematite that have undergone changes in crystal structure to accommodate one tightly bound molecule of water of hydration. Limonite will refer to minerals rich in goethite that also contain variable amounts of looselybound water included in the goethite lattice structure. Neither for laboratory samples nor for Martian surface materials will we be discussing pure minerals in the X-ray crystallographic sense.

Hovis' results show that we can immediately exclude magnetite as a principal iron oxide in the bright areas: its reflectivity declines slightly between 5000 and $6500 \AA$, a result independent of particle size. We next consider hematite and goethite, extrapolating Hovis' results a few hundred angstroms to derive a data point to compare with the $4500-\AA$ observations of Mars. The goethite sample studied had an impurity content of $5 \%$; the hematite $<1 \%$; and the magnetite, $10 \%$. We make our comparisons at the long wavelength $(6500 \AA)$ ends, where the laboratory curves for various particle sizes have maximum differences from one another. We select laboratory samples with particle sizes such that the absolute value of $\mathrm{K}$ agrees to within $20 \%$ of the observed value for the bright areas, and determine whether the observed wavelength dependence is reproduced. An example of such a fit is given in Figure 1. Somewhat bette $r$ agreement can be achieved by slightly lowering the mean particle size, but within our criterion the agreement is quite satisfactory. In Hovis' data, a sample of pulverized hematite that has appropriate $\mathrm{K}$ at $5500 \AA$ shows only a small change in reflectivity between 4500 and 6500 $\AA$. Sagan et al. (1965) have measured hematite powders that show much steeper reflectivity curves than that observed by Hovis, and that also have an acceptable value of $K$. However, the samples used by Sagan et al. were intentionally selected as naturally occurring terrestrial materials, while those of Hovis were especially prepared for high purity. It seems possible that goethite impurities in the hematite samples of Sagan et al. are responsible for the steep slope. 


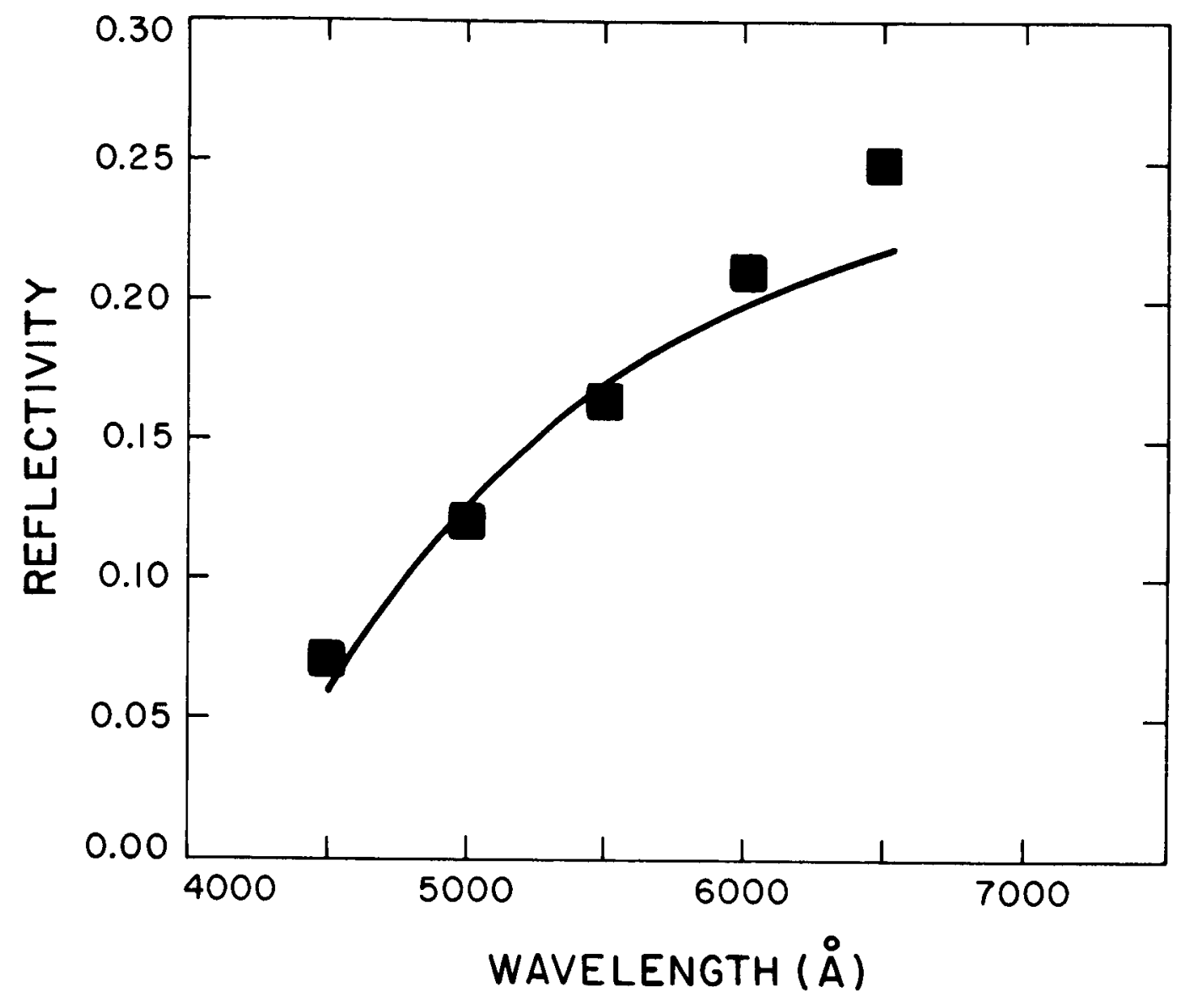

Figure 1. Comparison of the observed reflectivity, $K$, of the Martian bright areas (indicated by the squares) with the reflectivity of a laboratory sample of pulverized goethite (indicated by the solid line). 
On any planetary surface, we do not, of course, anticipate pure minerals. But the present discussion indicates that, of the iron oxides responsible for the gross visible photometric properties of Mars, magnetite and (probably) hematite make little contribution, while goethite and, possibly, limonite make major contributions.

Evans (1965) and Younkin (1966) report that between 3000 and $4500 \AA$ the reflectivity of Mars has a low and nearly constant value. This is in accord with laboratory measurements of samples of pulverized goethite and limonite, with particle sizes in the range discussed below.

\subsubsection{Determinations of particle sizes}

2.1.5.1 From K and $\Gamma$. The particle dimensions of laboratory samples (e.g., Hovis, 1965) are determined by attempting to pass the sample through a series of graded sieves. Thus a given particle radius, a, actually represents a distribution of particle radii between some upper limit and some lower limit. Quoted values of mean particle radii in this paper refer to median values when the distribution is closely bounded at both ends. When the lower end of the distribution function is distant, we will be concerned with cross-sectional averages. If the distribution function is $f(a)=a^{-n}$, where $0 \leq \mathrm{n} \leq 3$, then $\overline{\mathrm{a}}=[(3-\mathrm{n}) /(4-\mathrm{n})] \mathrm{a}_{\max }$, where $\mathrm{a}_{\max }$ is the largest par ticle radius. Thus, provided $\mathrm{n}$ is not too close to $3, \overline{\mathrm{a}}$ will be close to but somewhat less than ${ }_{\text {max }}$. To match the bright-area values of $K$ in the preceding section with laboratory samples of goethite, we require $\bar{a} \simeq 25 \mu$.

We next further test the hypothesis that the albedos of both dark and bright areas are controlled by iron oxides. In the process, we extract another value for the mean particle radius, $\bar{a}$. We find a value of $\bar{a}$ for which laboratory and Martian values of $\mathrm{K}(6500 \AA)$ agree to within $2.0 \%$ - but this time for the dark areas. One particle size of goethite satisfies the above requirements: $\overline{\mathrm{a}} \simeq 87 \mu$. 
We next attempt to match the observed values of $\Gamma$ with goethite samples of two different particle sizes, one for the bright areas (b), one for the dark (d). In Figure 2 we see that $\bar{a}_{b}=25 \mu$, and $\bar{a}_{d}=87 \mu$ satisfactorily match the $\Gamma(\lambda)$ observations. At $\lambda>6500 \AA$, the observed contrast curve also begins to flatten. Since $K(\lambda)$ for the bright areas agrees well with goethite or limonite (Figure 1), the agreement in Figure 2 indicates that goethite or limonite also controls the visual opacity of the dark areas. The $\Gamma(\lambda)$ curves also provide a very sensitive test of the correct choice of particle size, as can be seen from the very poor agreement of $\bar{a}_{b}=25 \mu, \bar{a}_{d}=175 \mu$ with the Martian contrast. It is because of this great sensitivity to particle size, and because introduction of a realistic particle-size distribution may somewhat change slopes, that we consider the agreement between the laboratory reflectivities for $\overline{\mathrm{a}}_{\mathrm{b}}=25 \mu, \overline{\mathrm{a}}_{\mathrm{d}}=87 \mu$ and the observed Martian reflectivities very satisfactory. These particle sizes assume the Martian surface is composed only of iron oxides. If there a re additional transparent substances, the actual particle sizes will be larger. The value of $\bar{a}_{d}=37 \mu$ matches the dark areas when they are not at their maximum darkening. Photometric studies by Focas (1961) give $\Gamma$ as high as 0.55 at $5800 \AA$ during the darkening maximum; such values of $\Gamma$ require $\bar{a}_{d} \simeq 200 \mu$ (cf. Figure 2 ). The possibility that the seasonal changes are due to a particle-size modulation by global winds will be discussed elsewhere.

One important further implication of Figure 2 is that goethite samples that match Martian $K$ and $\Gamma$ for bright and dark areas systematically lose contrast toward the blue. At $4500 \AA$, the contrast is $<10 \%$ and falls rapidly toward shorter wavelengths. Thus if iron oxides are the primary sources of opacity of the Martian surface, there will be an intrinsic loss of surface contrast in the blue and violet, and no need to postulate an atmospheric blue haze. Further discussion of the blue haze will be carried out below.

2.1.5.2 From fallout times of yellow clouds. We now attempt to check these particle sizes by independent methods, first by considering the dissipation times of yellow clouds on Mars. These clouds, with photometric properties similar to those of the bright areas, have typical lifetimes $\sim 1$ day (Slipher, 1962). A crude estimate of particle size can be obtained from this 


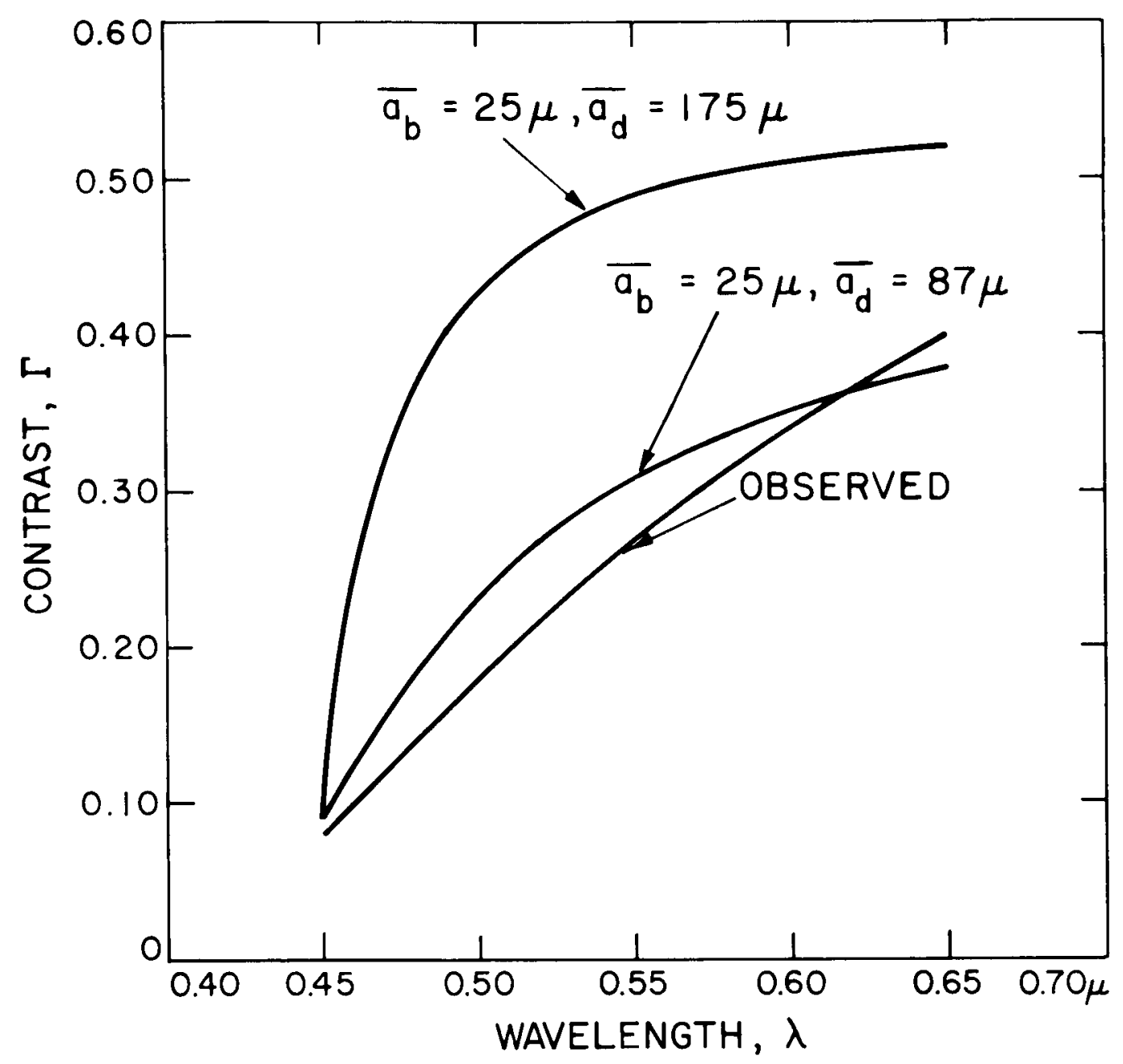

Figure 2. Comparison of the observed contrast between Martian bright and dark areas (lowest curve) with the laboratory contrast between different mean particle sizes of pulverized goethite. Two choices of the mean particle radius of the larger particles are shown.

Both laboratory curves show "bright-area" mean particle radii of $25 \mu$. The curves illustrate how sensitively the contrast depends on the choice of particle size. 
fallout time. We suppose the wind to lift particles off the bright areas to an altitude, $h$; at a time, $t$,later they fall out of the atmosphere. Under ae rodynamically smooth conditions - i. e., when the Reynolds number, Re, is small - the terminal velocity of a spherical particle is given by the StokesCunningham equation (see, e.g., Green and Lane, 1957). The fallout time is then

$$
t=\frac{h}{v}=\frac{4.5 \eta h}{\rho_{p} g a^{2}(1+\Lambda / a)},
$$

where $\eta$ is the dynamical viscosity, $\rho_{p}$ the density of the individual particles, $g$ the acceleration due to gravity, a the particle radius, and $\Lambda$ the atmospheric mean free path. Data tabulated by Goldstein (1938) on the drag coefficient as a function of the Reynolds number show equation (6) to be valid to better than $15 \%$ when $R e \leq 1$. If $\rho$ is the atmospheric density, $R e=v$ a $\rho / \eta$. For characteristic Martian surface conditions (say $\mathrm{T}=250^{\circ} \mathrm{K}$, and a $10-\mathrm{mb}$ surface pressure for an atmosphere composed of equal parts of $\mathrm{CO}_{2}$ and $\mathrm{N}_{2}$ ), $\eta=1.4 \times 10^{-4} \mathrm{gm} \mathrm{cm}^{-1} \mathrm{sec}^{-1}$ and $\mathrm{Re} \leq 1$ for $\mathrm{a} \leq 125 \mu$. We take $\rho_{\mathrm{p}}=3.8 \mathrm{gm}$ $\mathrm{cm}^{-3}$, the value for limonite; most other reasonable materials have similar values of $\rho_{p}$. Note that $\eta$ is independent of pressure and shows little dependence on temperature or composition. Under these conditions, we find $\Lambda=9 \mu$. By terrestrial analogy, h probably lies between $l$ and $10 \mathrm{~km}$ (see also de Vaucouleurs, 1954). For these two values of $h$, the computed values of $t$ for various choices of a are given in Table 2. The best choice of particle radius is evidently a round $12.5 \mu$; the value should be raised somewhat to allow for turbulent support of the particles. Note that because $\mathrm{t} \propto \mathrm{a}^{-2}$, approximately [equation (6)], the mean particle size of a dust cloud should be smaller than the mean particle size of the surface dust deposit that is the source of the cloud. Thus the result of $12.5 \mu$ from Table 2 is in good agreement with our estimates of $\bar{a} \simeq 25 \mu$ for the bright areas. We note that the yellow clouds cannot have mean particle radii much smaller than $\bar{a}$ for the bright areas; otherwise, the cloud will be white and not yellow. 
Table 2. Fallout times from altitudes, $h$, in the Martian atmosphere

\begin{tabular}{|c|l|l|}
\hline $\mathrm{a}$ & $\mathrm{h}=1 \mathrm{~km}$ & $\mathrm{~h}=10 \mathrm{~km}$ \\
\hline $5 \mu$ & $1.1 \mathrm{day}$ & 11 days \\
$12.5 \mu$ & 0.24 & 2.4 \\
$25 \mu$ & 0.068 & 0.68 \\
$50 \mu$ & 0.019 & 0.19 \\
\hline
\end{tabular}

2.1.5.3 From the rmal inertias. A third estimate of the mean particle size of the bright areas can be obtained from the bright-area the rmal inertia, as Leovy (1966) has pointed out. A first estimate, which is also an upper limit, to the thermal inertia may be found by comparing the observed infrared diurnal temperature variations with model calculations that neglect the temperature smoothing due to atmospheric heat transport. Sinton and Strong (1960) have made such measurements and performed such calculations. Unfortunately they have used too low a value for the bolometric albedo of the bright areas - 0.15 instead of about 0.25 (de Vaucouleurs, 1964; Walker, 1966) - and have not corrected for the effect of the finite aperture of their detector close to the limb, which results in an overestimate of the temperature near the limb. The first effect leads to a lowering by about $10^{\circ} \mathrm{K}$ of the theoretical temperature-time curves near local noon; with the appropriate albedo, the reciprocal the rmal inertia of the bright areas $(\kappa \rho c)^{1 / 2} \simeq 4 \times 10^{-3}$, in the usual units of $\mathrm{cal} \mathrm{cm}^{-2} \mathrm{sec}^{-1 / 2}$ (see Sinton and Strong's Figure 4). Here $\kappa$ is the the rmal conductivity of the surface, $\rho$ its density, and $c$ its specific heat capacity. Leovy has attempted to take account of the effect of atmospheric heat transport on the diurnal temperature variation. However, he commits the same oversight as Sinton and Strong, and also makes a linearization of the heat-balance equation that is very questionable for Mars.

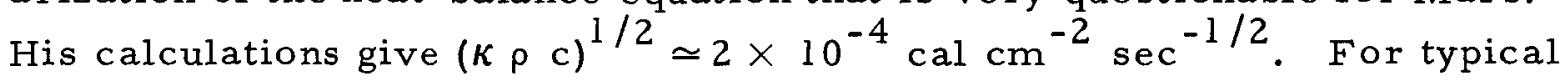
Martian pressures and such low values of the reciprocal thermal inertia, the thermal conductivity $\kappa$ becomes a function of the pressure and particle size and is virtually independent of composition (Leovy, 1966). Radar observations 
indicate that $\rho \simeq 1 \mathrm{gm} \mathrm{cm}^{-3}$ if the bright areas are composed mostly of limonite, and slightly higher values for other choices of composition (Sagan and Pollack, $1967 \mathrm{c})$. The specific heat is $0.22 \mathrm{cal} \mathrm{gm}^{-1}\left(\mathrm{~K}^{\circ}\right)^{-1}$ for limonite and for most other materials. The resulting values of $\kappa$ lie between $1.8 \times 10^{-5}$ and $7.3 \times 10^{-5} \mathrm{cal} \mathrm{cm}^{-1} \mathrm{sec}^{-1}\left(\mathrm{~K}^{\circ}\right)^{-1}$. The pressure over the bright areas probably ranges from 10 to $20 \mathrm{mb}$ (Sagan and Pollack, 1967b). From Leovy's Figure 3 it follows that the radii of particles in the Martian bright areas are $\bar{a}_{b} \leq 50 \mu$. The few points measured by Sinton and Strong for diurnal variations in dark-area temperatures give, as we expect, higher temperatures for the dark areas. This implies a similar the rmal inertia, thermal conductivity, and particle size for the dark areas, were the pressure there the same as in the bright areas. But Leovy's Figure 3 indicates that larger particles for the dark areas - as the photometric data imply - can be extracted if the dark-area pressures are significantly less than bright-area pressures. There is now a fair range of evidence that such pressure differentials do indeed exist (Sagan and Pollack, 1967b). But the infrared thermal data for the dark areas are not yet good enough to attempt putting the foregoing remarks on quantitative grounds.

Both cloud terminal-velocity and surface thermal-inertia estimates of $\bar{a}_{b}$ are consistent with the photometric estimates for pure goethite and with each other within their uncertainties. If iron oxides were a minor constituent of the Martian surface, the photometric particle radii for this composition would have been much larger, in gross disagreement with our two nonphotometric estimates of $\bar{a}_{b}$. Thus goethite or limonite must be a major constituent of the Martian bright areas. We have already argued that the dark-area composition must be closely similar. From the thermal-inertia calculations, the maximum value of $\bar{a}_{b}$ is comparable to the radius of pure goethite particles on the dark areas, again consistent with goethite as a major constituent of the dark areas. 
2. 1.6 A check on the photometric data

We are now in a position to perform a check on the photometric data of Dollfus, on which the foregoing discussion has been based. We will compare these data with independent photoelectric measurements of Martian geometrical albedos obtained by other observers. A number of photometric observations have been performed of the planet as a whole. Unfortunately it is not always possible to make direct use of such observations in the present context, since they refer to brightness averages of bright areas, dark areas, clouds, and polar caps. Particularly at shorter wavelengths, the reflectivity is raised significantly over the value expected for an ave rage of bright and dark areas because of the polar caps and limb clouds. At $\lambda<4000 \AA$, atmospheric Rayleigh scattering becomes important. Some of the observations employ fairly broadband filters; because the reflectivity of Mars is so sharply rising toward the infrared, a broadband measurement may not refer to the center of the bandpass.

We now connect these reflectivity observations with laboratory measurements. The geometrical albedo, $p$, is obtained from a measurement at $0^{\circ}$ phase angle of the total light reflected from Mars; it is related to the specific intensity by a relation similar to equation (4), but with I now denoting the specific intensity for backscattering with equal angles of incidence and reflection. Representing $I(\mu)=I(\mu=1) \mu^{\beta}$, analogous to equation (2), whe re the $\mu^{\prime}$ s are defined differently, we generate an equation analogous to (3); combining it with (3), we find

$$
R=\frac{2+\beta}{2+a} p
$$

The exponent $\beta$ may be determined from the observed limb darkening across the disk near $0^{\circ}$ phase angle. Here the data are plentiful. Again using Dollfus' (1957b) measurements, we find that a power law gives a good representation of the limb darkening, and that $\beta \simeq 0.25$. With our previous value of $a$, we find 


$$
\mathrm{p} \simeq 1.3 \mathrm{R}
$$

A similar relationship should hold for all small phase angles.

Instead of comparing $R$ with $p$ by equation (8), we check the value of $K$ obtained by Dollfus for the bright a reas (Table l) against values of $\mathrm{p}$ obtained from broadband photoelectric observations. From equations (5) and (8) the geometric albedo is connected with $\bar{K}$, the value of $K$ averaged over bright and dark areas, clouds, and the polar cap by

$$
p=1.1 \bar{K}=1.1\left(K_{b}-f K_{d}\right)+p_{c}=1.1 K_{b}(1-f \Gamma)+p_{c}
$$

where $f$ is the fraction of the surface area viewed that is made up of dark areas, and $\mathrm{p}_{c}$ is the contribution of clouds and polar caps in excess of the average contribution of bright and dark areas. Values for $\Gamma(4500 \AA)$ and $\Gamma(5500 \AA)$ will be taken from Table 1 ; they refer to the centers of prominent dark areas, not during the seasonal darkening. Values for $\Gamma(6900 \AA)$ and $\Gamma(7000 \AA)$ will be taken from data of Younkin (1966); they refer to the entirety of prominent dark areas during the seasonal darkening. Since the values of $\Gamma$ are weighted toward the darkest part of the planet, we choose $f \simeq 1 / 4$. Reasonable variations in this choice of $f$ will not affect our conclusions, except possibly at 6900 and $7000 \AA$. The contribution of the polar caps and clouds to $\mathrm{p}$ should be nearly $\lambda$-independent. Comparison of blue magnitudes in 1954, when there were both a southern polar cap and a variable northern polar cloud, with those of 1958, when there was only a variable northern polar cloud, gives a variation in p of about 5\% (de Vaucouleurs, 1964). This Martian cloudiness varied from night to night. Observations by Walker (1966) of $\mathrm{p}$ as a function of the central meridian longitude show $\mathrm{p}$ values in the blue between 0.072 and 0.091 . Part of this is due to a change in $f$ during the observing period. Thus $\mathrm{p}_{\mathrm{C}} \simeq 0.01$; the error in this estimate does not appear to be important, even in the blue. Finally, account must be taken of the broad passband, about $1000 \AA$, in the comparison we are about to make. When the reflectivity varies linearly with wavelength within the bandpass, the centroid of the bandpass will coincide with the effective wavelength, as 
is true at $5500 \AA$, and approximately so at 6900 and $7000 \AA$. However, in the blue the reflectivity is nearly constant at $\lambda<4500 \AA$ and rapidly increases at $\lambda>4500 \AA$, leading to a slight longward displacement of the effective wavelength. Since $\mathrm{K}$ changes by about 0.01 for each $100 \AA$ change in $\lambda$ above $4500 \AA$ (cf. Table 1), a displacement in effective wavelength by about $100 \AA$ implies the observed broadband $\mathrm{p}(4500 \AA)$ is raised by about 0.01 . While these estimates are crude, the correction factors will not alter the results in a major way. Collecting our correction factors for bandpass, polar caps, and clouds, and employing the equation above, we have solved for $\mathrm{K}_{\mathrm{b}}$ using photoelectric data on p compiled by de Vaucouleurs (1964). In Table 3 these results are compared with the conclusions of Dollfus that we have used above. The agreement is seen to be excellent.

Table 3. Comparison of bright-area reflectivities de rived by de Vaucouleurs (1964) and by Dollfus (1957b), equivalently reduced

\begin{tabular}{|c|c|c|}
\hline$\lambda$ & $\overline{\mathrm{K}}_{\mathrm{b}}$ & $\overline{\mathrm{K}}_{\mathrm{b}}$ \\
\hline & from de Vaucouleurs' $\mathrm{p}$ & from Dollfus \\
\hline $4500 \AA$ & 0.071 & 0.071 \\
5500 & 0.167 & 0.164 \\
6900 & 0.287 & 0.29 \\
7000 & 0.350 & 0.30 \\
\hline
\end{tabular}

\section{2 Inf rared Photometry and Its Implications}

\subsubsection{The "limonite band"}

We now compare laboratory spectra with Martian reflectivity measurements in the near infrared. Younkin (1966), Tull (1966), and Sinton (1967) have measured the disk-integrated reflectivity of Mars between 7000 and $11,000 \AA$. Laboratory spectra of oxides of iron show a halt to the rapid 
increase of reflectivity with wavelength at about $7000 \AA$; to a first approximation the spectra are fairly flat between 7000 and 11,000 . In many samples, a weak and broad absorption minimum is found, centered at about $9000 \AA$. Sagan et al. (1965) found this band for hematite, goethite, and limonite, some of these after dehydration. Apparently the band is independent of the degree of hydration and also independent of the presence of carbonates. This socalled limonite band the refore appears to be due to the ferric oxide moiety. The presence of this feature in Martian spectra should be independent of whether the ferric oxides are present as a thin patina on larger silicate particles, or as a major surface constituent. As long as oxides of iron are responsible for the visual and near-infrared opacity of the Martian surface material, this band should be present.

Figures 3 through 5 compare the reflectivities observed by Younkin, Tull, and Sinton, respectively, with the laboratory measurements on pulverized goethite that reproduced the visible reflectivity of the bright areas. At the times of measurement, the bright areas constituted about $75 \%$ of the area of the disk visible from Earth; thus comparison with the bright areas should be a valid first approximation. The Martian and laboratory curves have been normalized at $7000 \AA$. We see that both for Mars and for goethite the rapid rise in reflectivity with wavelength halts near $7000 \AA$ and that, to a first approximation, the reflectivity remains constant between 7000 and $11,000 \AA$. Tull finds a band centered at about 10,000 ; Younkin shows a hint of a weak band near $9000 \AA$, while a possible dip at about $8700 \AA$ appears in Sinton's spectra. We now discuss these results more fully.

The re are both intrinsic and observational problems connected with detection of the limonite band. The band itself is weak and quite broad - the reflectivity drops by only about $10 \%$ between 8000 and $9000 \AA$ for the goethite sample of Figures 3 through 5. Further, there is evidence that the depth, width, and wavelength of minimum reflectivity of the band differ from sample to sample for natural terrestrial varieties of limonite and goethite; e. g. , limonite obtained from Tuscaloosa, Alabama, exhibits a band centered at $9100 \AA$, while limonite pseudomorphs after pyrite obtained from Pelican 


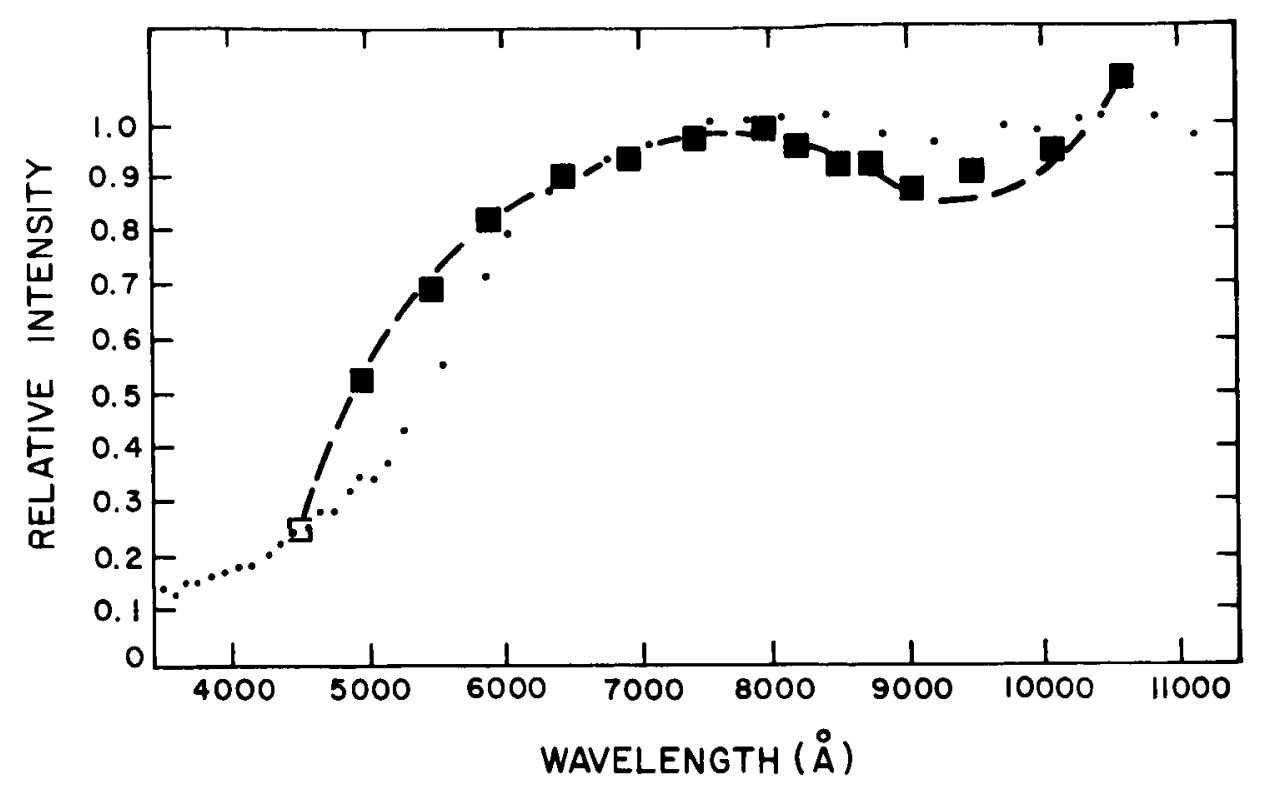

Figure 3. Comparis on of normalized reflectivity of the integrated Martian disk as a function of wavelength, determined by Younkin (1966) (filled circles), with laboratory data on goethite (filled squares).

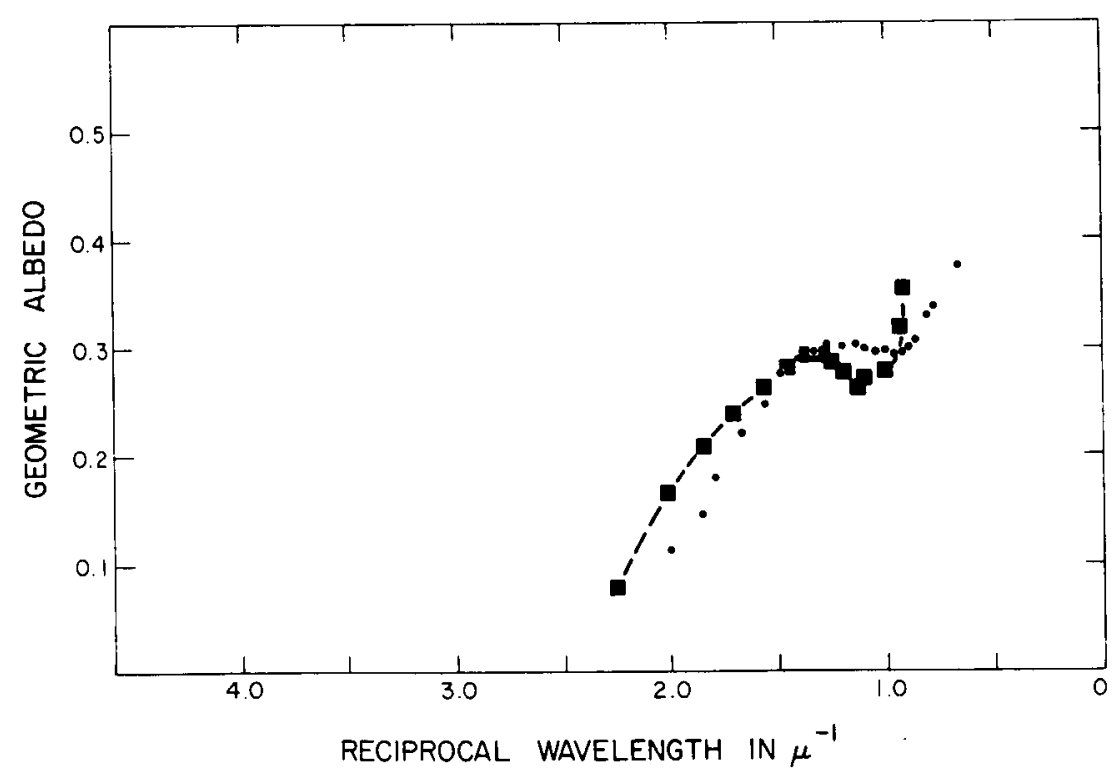

Figure 4. Comparison of the geometric albedo of the integrated Martian disk as a function of wavelength, determined by Tull (1966) (filled circles), with laboratory data on goethite (filled squares). 


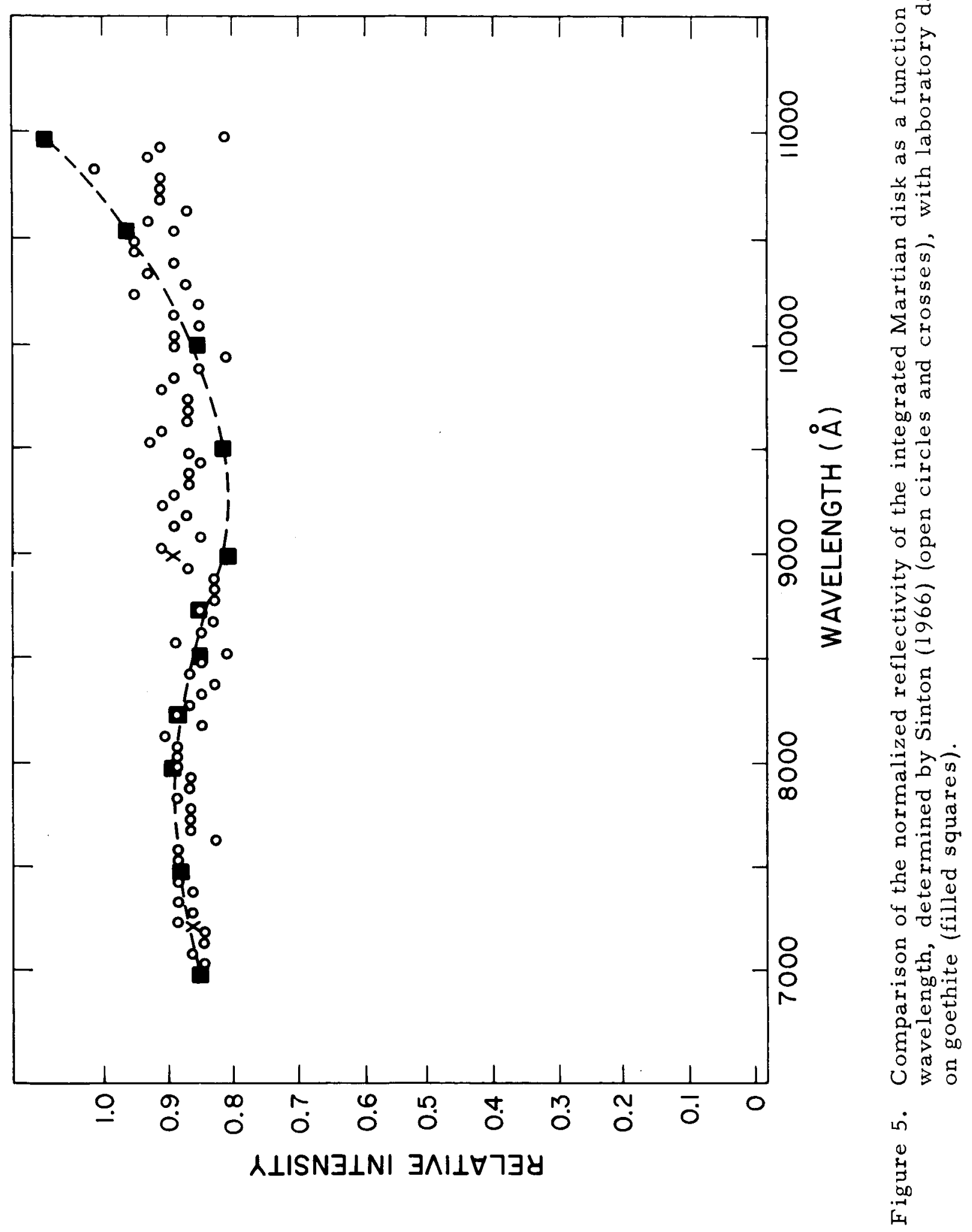


Point, Utah, exhibit a band centered at $8400 \AA$ (Sagan et al. , 1965). Hydrated ferric oxides over large regions of Mars should give a spectrum that is a blend of overlapping by mutually displaced absorption bands in this region; the presence of materials other than iron oxides will also tend to make detection of the band more difficult. For the goethite sample that matches the darkarea reflectivity in the visible, the band is located at $9700 \AA$. The presence of dark areas thus makes the band broader and more indistinct. Finally, we will below present evidence for the presence at 11,000 $\AA$ of a source of surface opacity other than iron oxides; any extension of this absorption to shorter wavelengths will further interfere with attempts to identify the limonite band.

On the observational side, the most serious problem is adequate solar comparison of the Martian reflection spectra. The discrepancies among the astronomical reflectivities summarized in Figures 3 to 5 indicate the magnitude of the problem. Younkin calibrated against the theoretical emergent spectrum of a Lyr, an AO star for which calculations at these wavelengths are questionable in part because of Paschen lines in the stellar atmosphere. Moreover, Younkin's calibration protocol, applied to othe $r$ stars and to the Moon, resulted in an anomalous drop in reflectivity near $9000 \AA$; Younkin was forced to make a correction of the same order of magnitude as the strength of the band being searched for. Younkin also made some lunar comparisons, but they a re discordant with the results of Tull. Tull calibrated against one locale on the Moon, and then converted to solar calibration using published measurements of the reflectivity of another locale on the Moon relative to the brightness of the Sun. Finally, Sinton calibrated against measurements of a G0 V star. For the broad and weak band searched for, the solar spectrum may differ significantly from that of a G0 V star.

It is extremely difficult to find convenient G2 V stars of sufficient brightness to use for such calibrations; but this would appear to be the most promising calibration procedure for further studies of the near-inf rared reflection spectra of Mars. Diffuse reflection of sunlight might also be employed. Alte rnatively, calibration could be made with a variety of late $F$ and early $G$ type stars. Because of the strong and variable absorption by telluric water vapor 
in this region of the spectrum, great care must be exercised in making corrections for extinction. It would also be helpful if improved topographical resolution were employed. It appears, despite the belief of Younkin and Sinton that the limonite band is not present on Mars, and despite the belief of Tull that it is prominent, that more work is needed to remove the existing ambiguities. But within the accuracy of measurement, these observations are not inconsistent with the conclusions of previous sections that goethite and limonite a re abundant on Mars.

\subsection{Composition of the Martian surface}

We now compare the contrast of bright and dark areas between 7000 and $11,000 \AA$ with laboratory samples, in order to test our hypotheses that ferric oxides are the opacity source in this wavelength region and that larger particles are present in the dark than in the bright areas. In Figure 6 are displayed measurements by Younkin (1966) of the relative reflectivities of the Aeria and Arabia bright areas and the prominent dark area, Syrtis Major. The curves show comparison with laboratory samples of goethite computed as before; the bright-area particles are assumed to have $\bar{a}_{b}=25 \mu$; the upper curve represents dark-area $\bar{a}_{d}=187 \mu$, the lower curve, $\bar{a}_{d}=87 \mu$, and the middle curve, an average of these extremes. Focas' (1961) photometry of Martian seasonal changes implies that Syrtis Major was experiencing a seasonal darkening at the time of Younkin's observations. From Figure 6 we see that $\overrightarrow{\mathrm{a}}_{\mathrm{b}}=25 \mu$ and $\overline{\mathrm{a}}_{\mathrm{d}} \simeq 140 \mu$ fit the observations quite adequately; the nearinfrared observations a re consistent regarding both composition and particle size with the conclusions previously drawn from visible-wavelength photometry.

Thus in three wavelength intervals, 3000-4500 7000-11,000 $\AA$, ferric oxides are capable of accounting for the spectra of both bright and dark areas. Beyond $1 \mu$, however, the opacity of ferric oxides becomes rather feeble, while other materials begin to absorb appreciably. Thus by comparing the observed Martian spectra at $\lambda>1 \mu$ with that of pure samples of ferric oxides, we may be able to infer the presence of other substances. Walker (1966) finds a geometric reflectivity of Mars at 


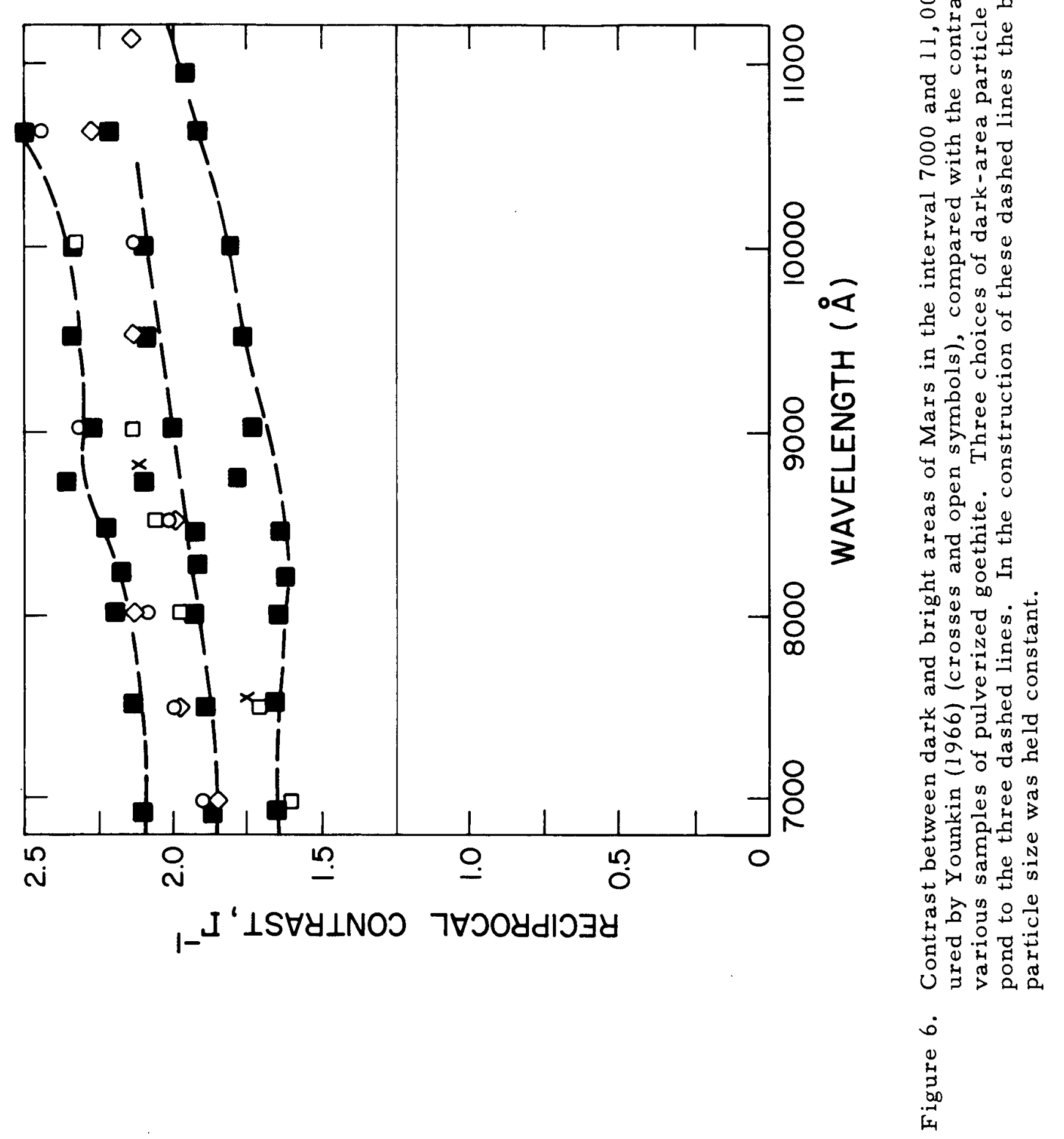


$1.63 \mu$ of 0.286 , va rying somewhat with the fraction of the disk covered by dark areas, but never exceeding 0.3. By equation (8), still assuming the limb darkening to be approximately wavelength independent, the laboratory reflectivity $\mathrm{R} \approx 0.22$. However, the relatively pure sample of goethite that accounts for the visible and near-infrared spectra of the bright areas shows $R(1.63 \mu) \simeq 0.42$. Similar difficulties are encountered with pure samples of hematite. The Stratoscope 2 (Danielson, Gaustad, Schwarzschild, Weaver, and Woolf, 1964) and previous ground-based (Kuiper, 1952) spectra imply at most a $40 \%$ rise in reflectivity between 1.0 and $1.4 \mu$ (Woolf, 1965), but the goethite and hematite samples show over a $100 \%$ reflectivity rise.

Studies on naturally occurring ferric oxides (Sagan et al., 1965) illustrate the influence that associated minerals can have on the spectrum at $\lambda>I \mu$. For example, a specimen of pulverized limonite from Cartersville, Georgia, shows only slightly higher reflectivity at $1.63 \mu$ than at $1 \mu$. The specimen contains $\mathrm{Fe}_{2} \mathrm{O}_{3}$ as a major constituent, but other constituents evidently are lowe ring the reflectivity between $1 \mu$ and $1.63 \mu$. At $\lambda<1 \mu$ this specimen behaves very like the pure goethite samples used in the earlier discussion. In a the rmogravimetric analysis, Sagan et al. heated their various samples in air to $1000^{\circ} \mathrm{C}$, driving of bound wate $\mathrm{r}$ and carbon dioxide present as carbonates, and then examined the reflection spectrum of the residues. Despite the fact that the residues were characterized in general by a larger mean particle size than the original samples, the residues often displayed a larger reflectivity at $\lambda>I \mu$. Because this phenomenon occurred for samples that showed no appreciable loss of gaseous material, we believe it is due to the oxidation of magnetite, $\mathrm{Fe}_{3} \mathrm{O}_{4}$, present as a constituent of the original samples, to $\mathrm{Fe}_{2} \mathrm{O}_{3}$. For the particle sizes of interest, magnetite absorbs very strongly beyond $1 \mu$ while $\mathrm{Fe}_{2} \mathrm{O}_{3}$ does not (Hovis, 1965).

It is reasonable that the iron on the Martian surface, like the iron in meteorites, has a mixed oxidation state. Because of the large opacity of magnetite, only a small fraction is required to reduce the goethite or limonite reflectivities beyond $1 \mu$. This circumstance also permits $\mathrm{Fe}_{2} \mathrm{O}_{3}$ to control the opacity at $\lambda<1 \mu$, which we have seen agrees very well with 
the data. Magnetite seems to offer the most reasonable source of additional opacity beyond $1 \mu$.

An alternative opacity source is the $\mathrm{MgO}$ moiety, present in meteorites and in the Earth's crust and mantle in abundance similar to that of silicates and of ferric oxides. Serpentine, $3 \mathrm{MgO} \cdot 2 \mathrm{SiO}_{2} \cdot 2 \mathrm{H}_{2} \mathrm{O}$, absorbs very strongly at $1.63 \mu$ and shorter wavelengths, but rather weakly below $1.0 \mu$ (Sagan et al., 1965). Carbonates are apparently only feebly absorbing, for the particle sizes of present interest, at $\lambda<3 \mu$ (Gray, 1963). Finally, if we estimate the strength of water-of-hydration bands from the tabulated absorption coefficients of ice (Irvine and Pollack, 1967), we find no significant contribution of the hydration band to opacity at $\lambda<1.45 \mu$, and thus could not explain the fact that the Martian reflectivity rises only slightly between 1.0 and $1.4 \mu$.

Walker (1966) finds $\Gamma(1.63 \mu)$ to be large, the dark areas having about half the reflectivity of the bright areas. But assuming (1) the goethite required for the optical-frequency dark-area reflectivity and (2) that goethite is the only opacity source, we find that the predicted $1.63-\mu$ reflectivity of the dark areas somewhat exceeds the observed reflectivity of the bright areas. We conclude that the dark areas as well as the bright areas need an additional opacity source such as $\mathrm{Fe}_{3} \mathrm{O}_{4}$ or $\mathrm{MgO}$ for $\lambda>1 \mu$.

\section{2. 3 Bound-water content of the Martian surface}

Water of hydration, if present in the superficial layers of the Martian surface, will contribute to the absorptivity at $\lambda \geq 1.45 \mu$. When this opacity source is added to that of $\mathrm{Fe}_{2} \mathrm{O}_{3}$, which decreases steadily in absorptivity for $\lambda>1 \mu$, we see that a reflectivity peak near $1.4 \mu$ is expected for hydrated ferric oxides. Such a peak is in fact observed between 1.3 and $1.45 \mu$ in a range of samples of goethite and limonite (Sagan et al. , 1965; Hovis, 1965). Hematite, in contrast, shows a slow monotonic reflectivity increase for $\lambda>1 \mu$; some hematite samples with magnetite as a probable impurity reach a reflectivity plateau for $1.00 \mu \leq \lambda \leq 1.18 \mu$ (Sagan et al. , 1965; Hovis, 1965). The Stratoscope 2 data plus the observation that Martian 1- $\mu$ and 2- $\mu$ reflectivities are very nearly equal (Kuiper, 1952) point to a peak in the Martian 
reflection spectra near 1.4 (Woolf, 1965); this would seem to imply water of hydration on Mars.

Sinton (1967) has apparently detected bound water in both bright and dark areas, through measurement of a sharp drop in reflectivity between 2.4 and 3. $0 \mu$. Figures 7 and 8 display his observations for regions consisting primarily of bright areas and of dark areas, respectively. Bound water has a strong absorption band centered at $3.1 \mu$.

However, before accepting the reflectivity decline between 2.4 and $3.0 \mu$ as diagnostic for water of hydration, we explore several other possibilities. Ice has a strong band centered at $3.1 \mu$; conceivably a permanent haze layer of ice crystals in the Martian atmosphere could be responsible for the observed reflectivity. Since Sinton observed only regions near the center of the disk, we can exclude the polar regions and limb clouds from consideration. For typical ice particles, $1 \mu \leq a \leq 100 \mu$, complete absorption will occur around 3 . $1 \mu$; and $\tilde{\omega}_{0}$, the single scattering albedo, will be $1 / 2$ when account is taken of the diffracted component of scattering (Sagan and Pollack, $1967 \mathrm{a})$. The observed reflected light will then be almost entirely due to a double traversal of the clouds and intermediate reflection from the planetary surface. Thus the clouds reduce the intensity that might otherwise be seen by $\exp \left[-2\left(1-\tilde{\omega}_{0}\right) \tau_{1}\right] \simeq \exp \left(-\tau_{1}\right)$ for vertical incidence, where $\tau_{1}$ is the interaction optical depth of the clouds. To reduce the reflectivity by about a factor 3 , as the observations demand, $T_{1}$ must be about 1 . If the ice crystals have $a \geq 15 \mu$, and $\tau_{1}=1$, the re would be more condensed water in the clouds than water vapor in the underlying atmosphere - a most unlikely circumstance. From formulae developed elsewhere for anisotropic nonconservative scattering by ice crystals (Sagan and Pollack, 1967a), we find that clouds with $\tau_{1} \simeq 1$ lead to an enhancement of the visual Russell-Bond albedo by 0.15 for $15-\mu$ particles and by larger amounts for $a<15 \mu$; at visible wavelengths, absorption by ice clouds is negligible. Such an increment is clearly too high. The Russell-Bond albedo at $3600 \AA$ is 0.043 (Walker, 1966), and most of this is very likely reflected light from the surface. We conclude that possible 

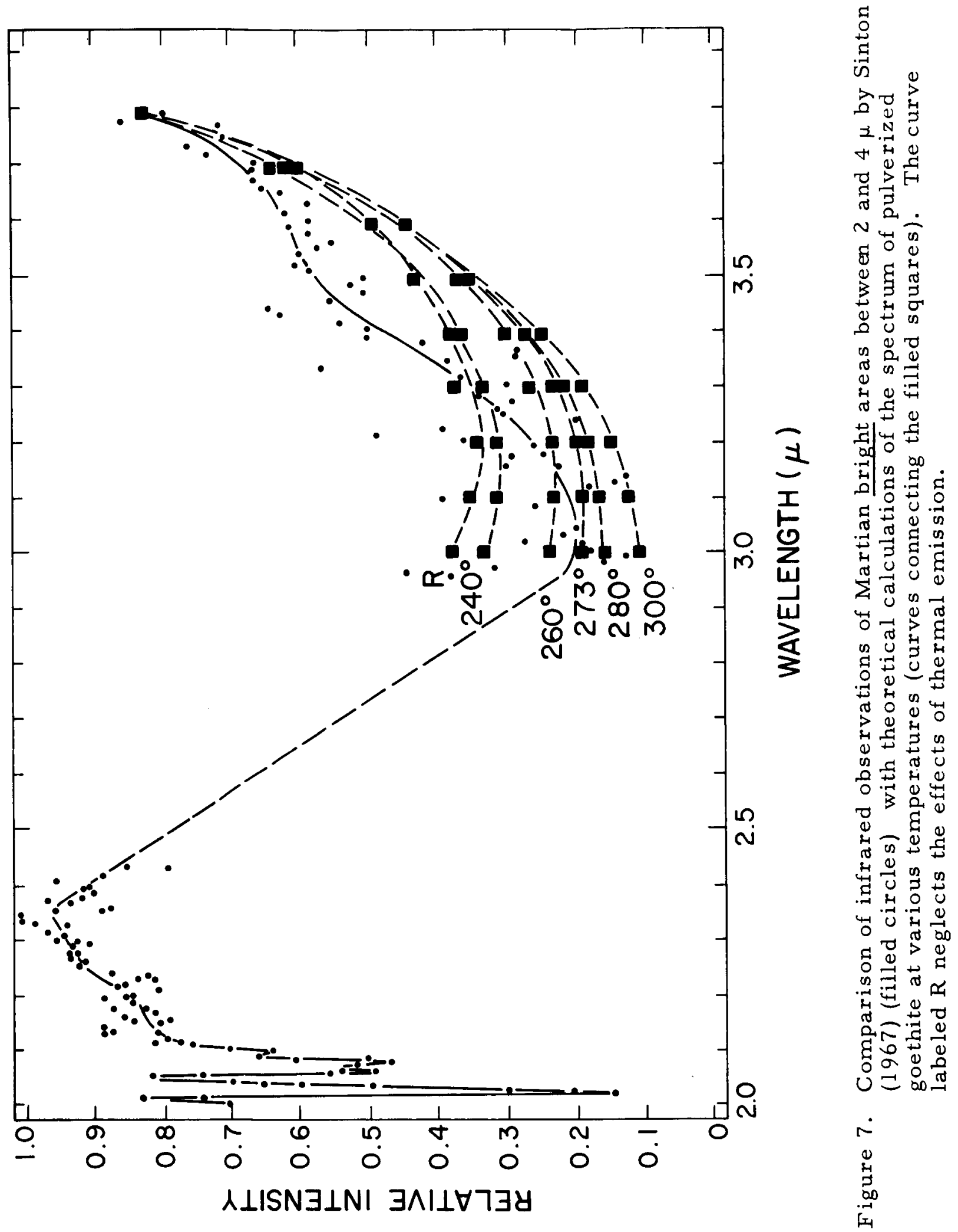


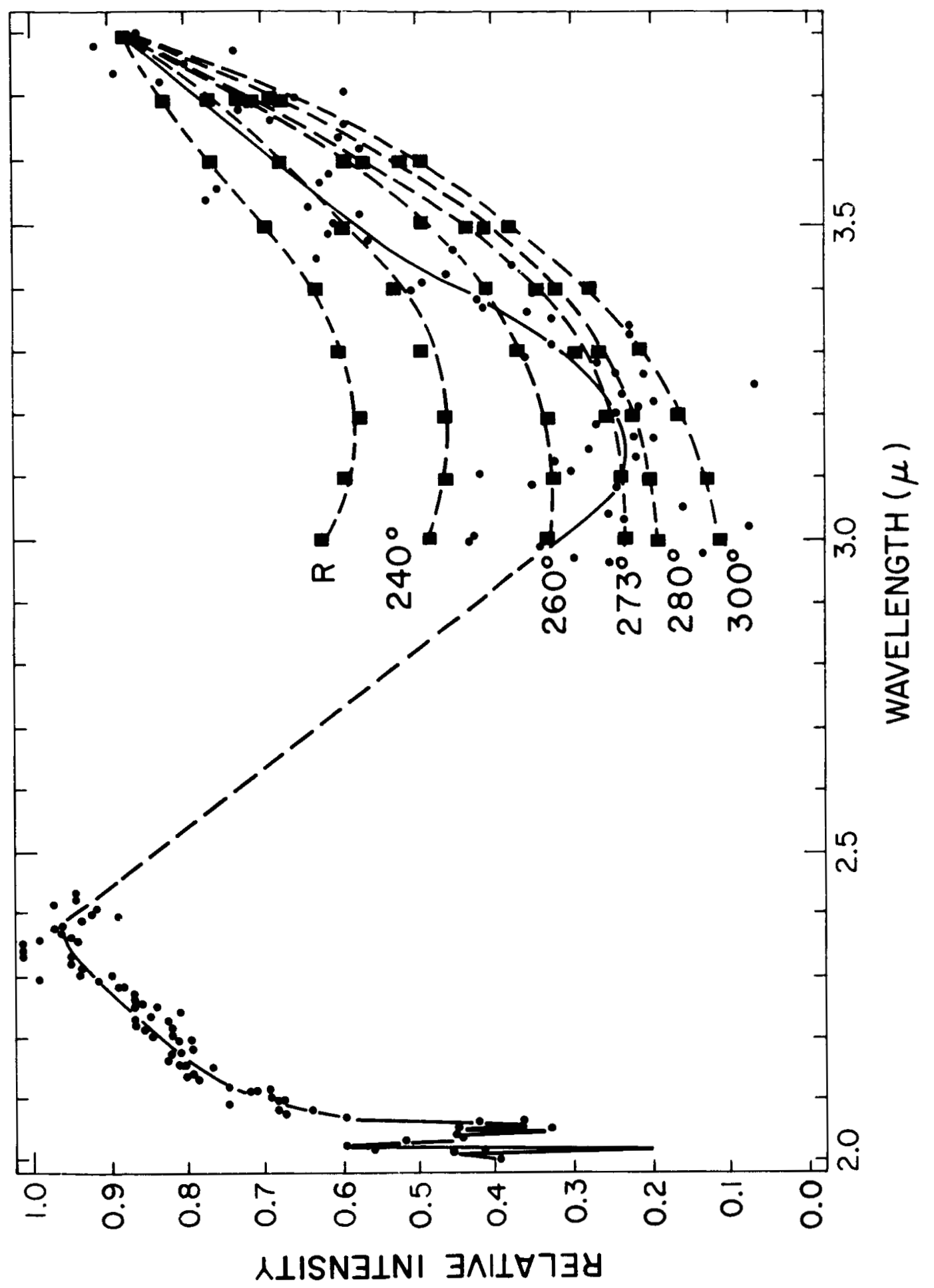

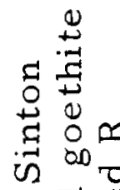

ล

그응

小

를

N

(1)

() उี

उ出

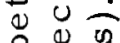

a 0

of is 4

过空

(6)

क्ष

尔

인

舫

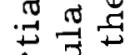

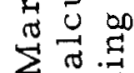

出

0 药

告

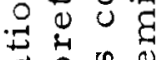

$>00$

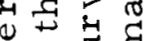

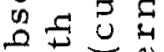

0 점

虫 $\stackrel{4}{3}$

त $0 \begin{aligned} & \text { त } \\ & 4\end{aligned}$

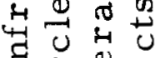

$\exists \stackrel{0}{ } \pm$

पै U द्व

ᄃ

$0 \cong$ 叫

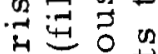

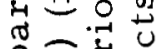

द्विर क

$\cup \Xi \leftarrow \stackrel{0}{0}$

$\infty$

岂 
ice-crystal hazes on Mars, of whatever particle size, cannot account for Sinton's observations.

A second pos sibility is that the observed dip is due to a mineral marked by strong absorption in the 3 - to $4-\mu$ region; the most geochemically abundant such materials, and the most plausible in a Martian context, are the carbonates. However, the carbonate band is centered some $0.4 \mu$ to $0.6 \mu$ longward of the observed dip center near 3.0 $\mu$. Even if thermal emission at $\lambda>3.4 \mu$ were to cause an apparent shift of the wavelength of minimum intensity, this explanation would remain untenable. Even if we assume as much calcite as iron oxides on Mars - a generous assumption, since we have shown iron oxides to be a principal constituent of the surface - we calculate from the absorption coefficients tabulated by Gray (1963) that carbonates will absorb only $\sim 0.01$ of the light traversing a particle in the bright areas and $\sim 0.05$ for a dark-area particle.

We now estimate the quantity of bound water. The reflectivity $R(3.1 \mu)$ can be estimated as follows: from Sinton's curves, $R(3.1 \mu) / R(2.2 \mu) \simeq 0.29$ for the dark areas and 0.24 for the bright areas. Walker (1966) finds an average value for the geometrical albedo $\mathrm{p}(2.2 \mu)=0.26$. This may be taken as representative of the bright areas. With an aperture comparable to Sinton's, Walker finds that when a region encompassing primarily dark areas is viewed, the reflectivity drops by a factor of about 2; this factor is only approximate, and varies with the dark area viewed. With these data and equation (8), we find $R_{d}(3.1 \mu) \simeq 0.029$ and $R_{b}(3.1 \mu) \simeq 0.052$; these values are somewhat uncertain owing to both the scatter in Sinton's data set and the variation of $R_{d}(2.2 \mu) / R_{b}(2.2 \mu)$. The small contribution from the rmal emission at $3.1 \mu$ will increase the apparent value of $R(3.1 \mu)$ somewhat above the true value for reflection only. After correction for emission is made, a very prominent 3- $\mu$ absorption band, intrinsic to the Martian surface, remains. The samples of goethite that match the visual reflectivities of the dark and bright a reas show almost complete saturation of the absorption band; the reflectivities are $R_{d}(3.1 \mu) \simeq 0.029$ and $R_{b}(3.1 \mu) \simeq 0.030$. Thus, the Martian hydration bands seem close to saturation. 
A better estimate of the relative water content can be made by comparing computed and observed spectra in the 3.0- to $3.8-\mu$ region. The goethite sample that matches the visual reflectivity of the bright a reas shows a significant increase in reflectivity between 3.0 and $3.8 \mu$. The corresponding sample for the dark areas shows only a very slight such increase; he re the particle size and therefore the mean bound-water content per particle are larger, and the absorption remains close to saturation out to $3.8 \mu$.

In these calculations we must take explicit account of the rmal emission, which must certainly be responsible for at least part of the reflectivity rise at $\lambda>3 \mu$. Sinton and Strong (1960) have measured temperatures of bright areas near the center of the disk of some $300^{\circ} \mathrm{K}$ for the nearly perihelic opposition of 1954. Sinton's (1967) infrared spectra were obtained during the more aphelic opposition of 1963; because of the eccentricity of the Martian orbit, the comparable temperature should be about $280^{\circ} \mathrm{K}$. Sinton's spectrometric aperture was about half the size of the Martian disk. The radiometric measurements of 1954 also give the variation of temperature with equatorial longitude, and imply a mean temperature of $270^{\circ} \mathrm{K}$, approximately, over the aperture of the 1963 spectra. Dark areas exhibited temperatures some $8^{\circ} \mathrm{K}$ warmer than neighboring bright areas; we adopt $275^{\circ} \mathrm{K}$ as a mean temperature over the aperture for the dark areas. Thermal emission and reflected sunlight may be combined analytically by adding blackbody specific intensities to the reflected specific intensities obtained from equation (3). The laboratory goethite curves supply the 3.0 - to $3.8-\mu$ true reflectivities, under the as sumption, already discussed, that absorption by other materials is not important in this wavelength range. Figures 7 and 8 show the computed curves for various temperatures, as well as the laboratory curve, $R$, for no contribution from thermal emission. The calculations have been normalized to agree with the observed data point at $3.8 \mu$, whe re the observational scatter is relatively small.

For the expected temperatures $-270^{\circ} \mathrm{K}$ for Figure 7 and $275^{\circ} \mathrm{K}$ for Figure 8 - the computed dark-area curve agrees with observation, within the observational scatter, while the computed bright-area curve (Figure 7) 
lies below the data points between 3.4 and $3.8 \mu$. One possible cause for this disagreement is that the true bright-area reflectivity as sumed, given by curve $R$, is dropping too rapidly at $\lambda<3.8 \mu$; i. e., less than full saturation of the bound-water band occurs at these longer wavelengths. The agreement is better for the dark areas (Figure 8) because the water band is more nearly saturated. Note that, while Sinton has fit the steep solid line to his 3.1 - to 3. 4- $\mu$ observations, the scatter in the data points clearly admits the computed

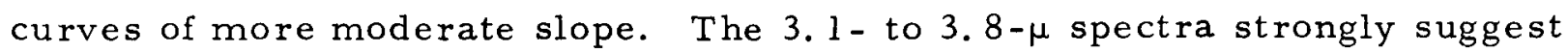
that the Martian dark areas have at least one molecule of water per molecule of ferric oxide, as is true for the nearly saturated goethite curves used in the calculations. The bright areas must have nearly as much bound water. If there were less water, the absorption bands would be less saturated, and the computed curves for both bright and dark areas would fall significantly below the data points.

The density of water in goethite where $\left(\mathrm{Fe}_{2} \mathrm{O}_{3}\right) /\left(\mathrm{H}_{2} \mathrm{O}\right) \approx 1$ is $0.4 \mathrm{gm} \mathrm{cm}^{-3}$. Since a significant fraction of the Martian surface material is composed of such material, the density of bound water in the surface layers is not much below that in pure water. This water is held with a bond energy of a few Kcal per mole. Geothermal activity on Mars should be able to release substantial quantities of bound water into the atmosphere. Further, if hypothetical Martian organisms are capable of enzymatically breaking the weak hydration bonds, there may be no dearth of water at all for Martian biology. While the Martian surface is not wet, it may nevertheless be aqueous.

\section{2. 4 The rmal emission and composition}

Spectroscopy of Martian thermal emission between 8 and $13 \mu$ has been performed by Sinton and Strong (1960). Figure 9 shows the observed intensity per unit wavelength interval, the product of the blackbody specific intensity and the emissivity. Deviation of the observed spectrum from that computed on the assumption of constant emis sivity fixes the wavelength variation of emissivity and may provide some information on composition. The appropriate mean temperature is computed as follows: the blackbody emission in the 


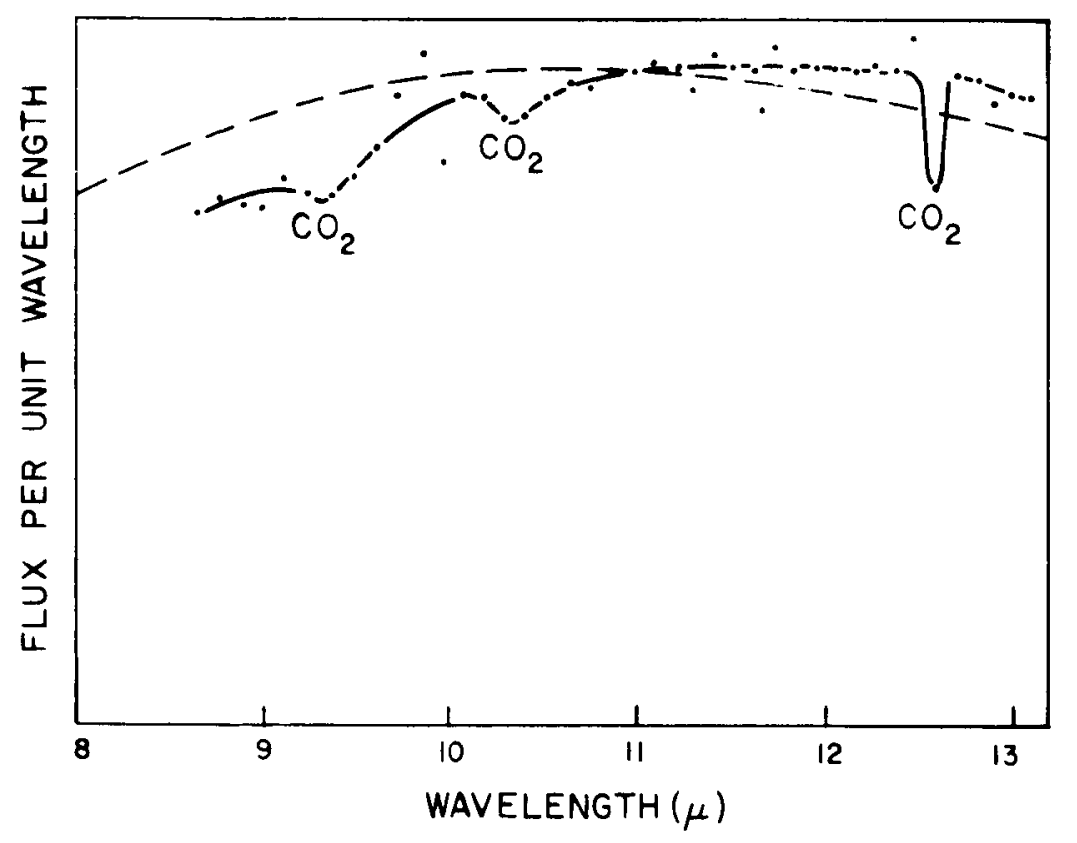

Figure 9. Comparison of spectral observations of Mars in the thermal infrared (Sinton and Strong, 1960), given by the dots, with a theoretical blackbody spectrum, given by the dashed line, at a temperature of $273^{\circ} \mathrm{K}$. The two curves have, arbitrarily, been made to agree at 1l- $\mu$ wavelength. The differences between the curves - apart from atmosphere absorption - may indicate emissivity variations of the Martian surface material. 
8 - to 13- $\mu$ region will be represented (cf. Pollack and Sagan, 1965b) by a powerlaw dependence on temperature, $\mathrm{B}_{\lambda} \propto \mathrm{T}^{\mathrm{n}}$. We wish to derive the temperature, $<\mathrm{T}>$ of an equivalent isothermal disk that generates the same specific intensity, integrated over the relevant fraction of the disk, as is produced by the actual distribution of surface temperature. Specific intensity is averaged by a solid angle weighting, and azimuthal symmetry is assumed. We then find

$$
\langle\mathrm{T}\rangle=\mathrm{T}_{0}\left[2 \int_{0}^{1}\left(\mathrm{~T} / \mathrm{T}_{0}\right)^{\mathrm{n}} \mu \mathrm{d} \mu\right]^{1 / \mathrm{n}}=\left(2 \int_{0}^{1} \mathrm{~T}^{\mathrm{n}} \mu \mathrm{d} \mu\right)^{1 / \mathrm{n}} .
$$

In equation (9), $\mathrm{T}_{0}$ is the temperature at the center of the disk, and arccos $\mu$ is the angle between the local normal and the direction to the Earth, or, equivalently, the direction to the Sun for the small phase angle of observation. We assume $\mathrm{T}=\mathrm{T}_{0} \mu^{1 / 4}$; i. e., the temperature is determined primarily by the insolation, and the surface is a poor conductor. With the appropriate value of $\mathrm{n}$ for these wavelengths and temperatures, and $\mathrm{T}_{0} \simeq 300^{\circ} \mathrm{K}$ for these observations, we find $\langle\mathrm{T}\rangle \simeq 273^{\circ} \mathrm{K}$.

Figure 9 displays the fit of the computed flux curve, given by the dashed line, under the assumption of constant emissivity, to the observations. We see that there is little variation of emissivity over the 8- to $13-\mu$ region, with no more than an increase in emissivity of 10 to $15 \%$ occurring between 8.5 and $13 \mu$. Because of the uncertainties in our knowledge of the exact temperature distribution, even this small variation may be spurious. It was after an argument of essentially this sort that Sinton and Strong concluded that the abundance of silicates on Mars is very low: solid quartz exhibits an emissivity of about 0.15 at $8.8 \mu$ due to restrahlen bands, going rapidly to unity at other wavelengths.

Laboratory measurements of silicate reflectivity by Hovis and Callahan (1966) show this conclusion to be premature. Their reflectivities can be converted to emissivities by Kirchhoff's law. Since Hovis and Callahan used only one angle of incidence in their measurements, their reflectivities will not be rigorously appropriate angular averages; however, a discussion along 
the lines of that in Section 2. 1.4 reveals that the error should be quite small. In common rocks with large silicate abundances, the reflectivity peak is considerably broadened; consequently, the peak reflectivity is less than that of, say, pure quartz. For example, in granites with a silicate abundance of over $65 \%$, the peak reflectivity is only slightly above $20 \%$, compared with a peak value of $85 \%$ for $\mathrm{SiO}_{2}$. Further, the wavelength of peak reflectivity increases as the rock becomes more basic, i.e., has less silicate. Dunite, which has a silicate abundance $<45 \%$, peaks at $11 \mu$. For these rocks, reflectivity maxima occur where $\left|\mathrm{n}_{\mathrm{c}}-1\right|$ is a maximum; as previously discussed, such marked wavelength variation in $\left|n_{c}-1\right|$ occurs in the middle inf rared because here $\mathrm{n}_{\mathrm{i}} \sim \mathrm{n}_{\mathrm{r}}$

Pulverization of the rock may either increase or decrease the reflectivity. An increase in reflectivity occurs by the phenomenon we have already discussed - an increase in multiple scattering of transmitted photons. In the middle infrared, new reflectivity maxima will arise at wavelengths different from those for larger particle sizes, and at such wavelengths that $n_{i}$ is a minimum.

As pulverization continues, some of the particles will have dimensions smaller than a wavelength; such particles are very poor scatterers and become near-perfect absorbers (cf. van de Hulst, 1957) - the net reflectivity then approaches zero. For this reason van Tassel and Salisbury (1964) found little wavelength variation of emissivity for finely ground quartz at 8 to $13 \mu$, and were able to conclude that powdered quartz was not inconsistent with the spectra of Sinton and Strong.

The foregoing discussion permits us to refine this conclusion somewhat. Sinton and Strong's spectra are compatible with significant amounts of fine quartz mixed with ferric oxides, for powders with particles in the size range previously deduced. Red sandstone powders with $\bar{a} \sim 100 \mu$ exhibit a 5\% increase in emissivity between 8 and $13 \mu$ (Hovis and Callahan, 1966). The dilution of silicates with ferric oxides, the broadening of the $\mathrm{Si}-\mathrm{O}$ restrahlen band, and the pulverization of the sample all contribute to the small emissivity variation. Powdered granite with $\bar{a} \sim 100 \mu$ shows an 
emissivity increasing with wavelength between 8 and $13 \mu$ by about $10 \%$, again compatible with the observations. On the other hand, powdered dunite has a peak reflectivity at $11 \mu$, which seems incompatible with the data of Sinton and Strong. Finally, pure quartz particles with $\overline{\mathrm{a}} \lesssim 10 \mu$ would also exhibit little emissivity variation; however, these particles may be too small, compared with our estimates of bright-area particle sizes above. In any case, the 8- to 13- $\mu$ emission spectra of Mars are consistent with large amounts of surface ferric oxides, but do not place very stringent constraints on the silicate abundance. 


\section{POLARIMETRY OF THE BRIGHT AND DARK AREAS}

\subsection{Nature and Reliability of the Data}

A largely independent source of information on the structure and composition of the Martian bright and dark areas lies in data on the polarization of initially unpolarized sunlight reflected from Mars. Figure 10 exhibits typical polarization curves, obtained by Dollfus (1957a). Curves $b^{a}$ and $b^{\beta}$ refer to bright areas, curve $d^{\alpha}$ to dark areas. They plot linear polarization against phase angle. The polarization is considered positive when the intensity of the component having its $E$-vector perpendicular to the ecliptic plane is dominant, and is defined as

$$
P \equiv \frac{I_{\perp}-I_{\|}}{I_{\perp}+I_{\|}} .
$$

Current techniques measure this polarization, $P$, to bette $r$ than $0.1 \%$. The phase angle, $\Phi$, is the angle between the Earth and the Sun, as viewed from Mars. Because of the relative geometries of the orbits of Earth and Mars, only those values of $\Phi$ between 0 and about $45^{\circ}$ can currently be observed. With space vehicles, measurement of $P$ over the full range $0^{\circ} \leq \Phi \leq 180^{\circ}$ becomes pos sible.

Before discussion of the implication of these data it is important to assess their reliability. Figure 11 shows the actual data points from which curves $b^{a}$ and $b^{\beta}$ of Figure 10 were constructed (Dollfus, 1957a). Curve $\mathrm{b}^{\mathrm{a}}$ was obtained from data gathered during the 1952 and 1954 oppositions; curve $b^{\beta}$ is for 1948 and 1950 . The measurements refer to bright areas near the center of the disk. For the 1948 and 1950 oppositions such areas were at a latitude of about $20^{\circ}$; for the 1952 and 1954 oppositions, about $0^{\circ}$. 


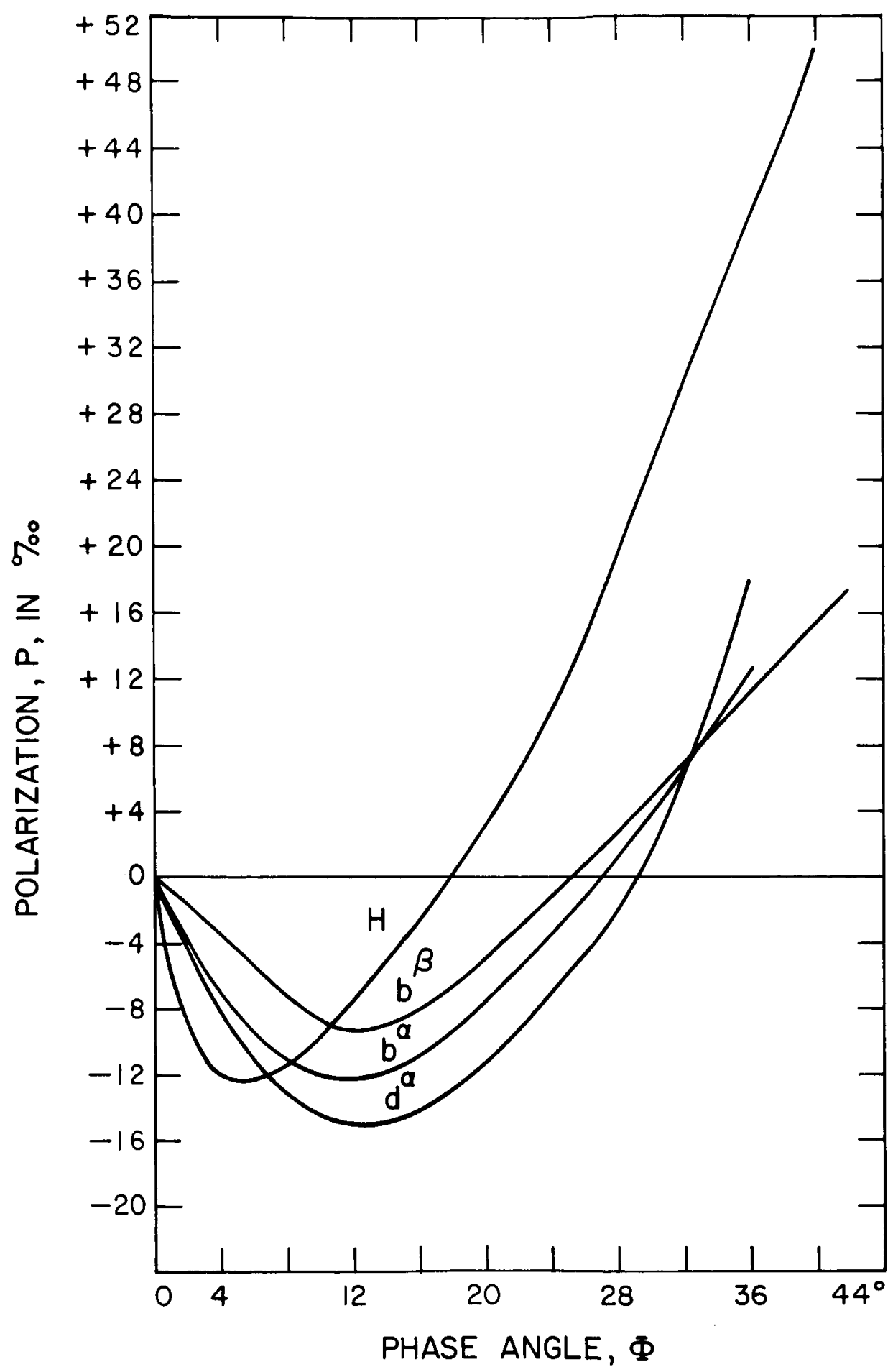

Figure 10. Comparison of polarization curves for bright and dark areas of Mars with the corresponding curve for pulverized hematite (curve H). After Dollfus (1957a). 


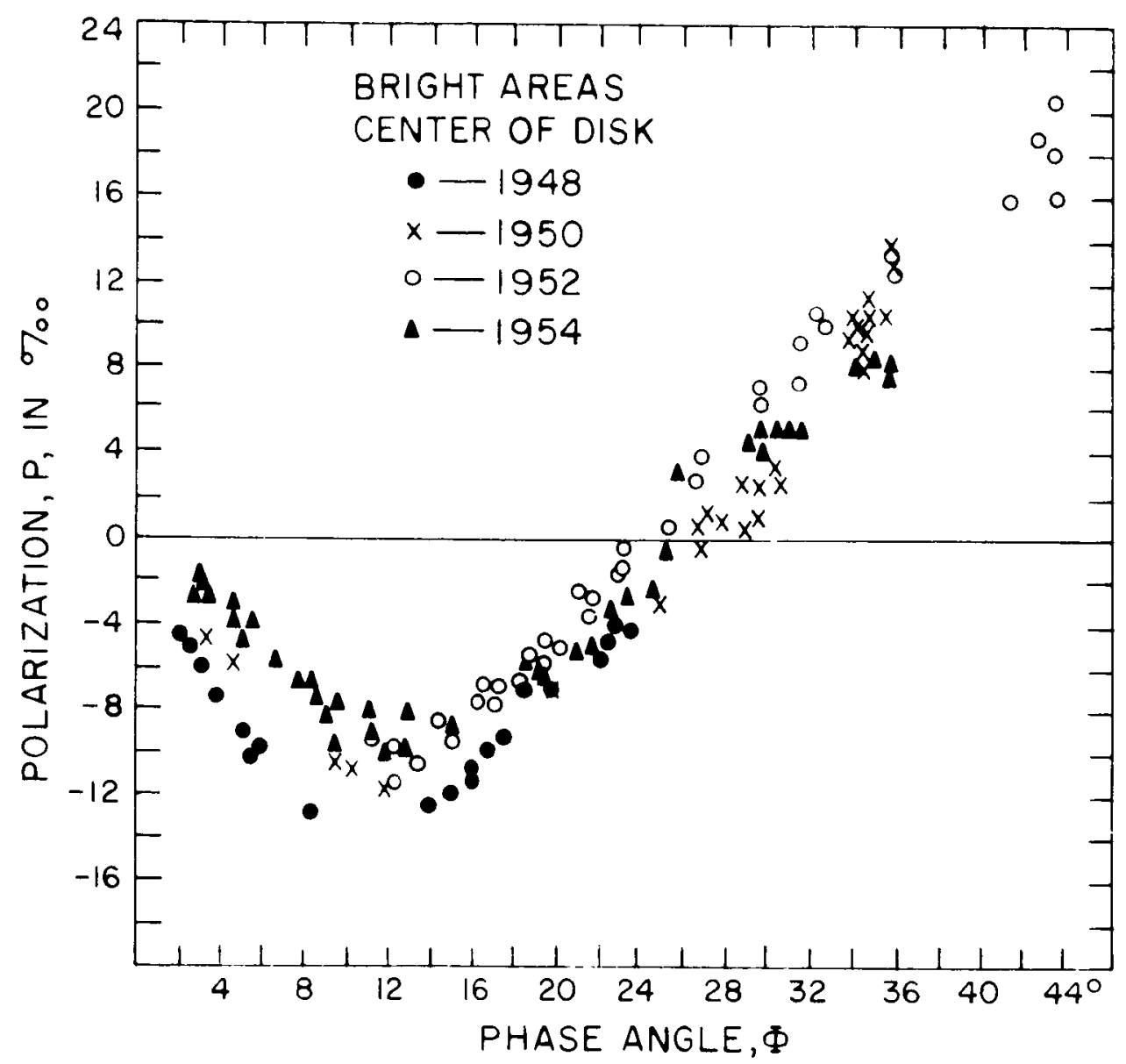

Figure 11. Polarization curves for Martian bright areas near the center of the disk for the four oppositions 1948, 1950, 1952, and 1954. These are the data points on which the curves labeled $b$ in Figure 10 were constructed. After Dollfus (1957a). 
Furthermore, the $b^{a}$ data pertain to local spring in the Northern Hemisphere, the $b^{\beta}$ data to local summer. It is reasonable that $b^{a}$ and $b^{\beta}$ should differ somewhat. Comparing Figures 10 and 11 , we conclude that there is not a large amount of scatter in the data points, and that the P- $\Phi$ curve is reasonably well defined. The phase angle of the negative minimum, its polarization value, and the phase angles for $\mathrm{P}=0 \%$ can all be obtained fairly accurately. The slope of the positive branch is known with somewhat less accuracy. Figure 12 displays polarization data obtained 15 years later when the configuration of the Sun, Mars, and Earth was similar to that in 1948 and 1950 (Dollfus and Focas, 1966). The data points of Figure 12 are reasonably consistent with the general characteristics (such as the second zero in the polarization curve) of curve $b^{\beta}$, particularly so since different bright areas were viewed. The points on the left half of Figure 12 correspond to the 1948 observations, those on the right to the 1950 data. Furthermore, photoelectric polarimetry by Morozhenko (indicated by the open deltas) agrees well with the visual polarimetry of Dollfus and Focas. In summary, the polarization data show both internal and external consistency.

\section{2 Influence of the Martian Atmosphere on the Polarization Curves}

The observed polarized light arises from both the Martian atmosphere and the Martian surface. To study the surface contribution, we must first subtract out the atmospheric contribution; it can arise both from Rayleigh scattering by gases and from Mie scattering by aerosols. We note that Dollfus' measurements refer in general to times when no obvious clouds can be detected over the area observed.

Estimates of the atmospheric polarization, assuming the entire contribution to be from Rayleigh scattering, led Dollfus (1957a) to an initial estimate of $85 \mathrm{mb}$ for the surface pressure, with a stated uncertainty of about a factor 2. Since this pressure is much higher than the values subsequently deduced from ground-based infrared spectrometry and from the Mariner 4 occultation experiment, there is an apparent implication of a substantial aerosol contribution to the atmospheric polarization (Kuiper, 1964). 


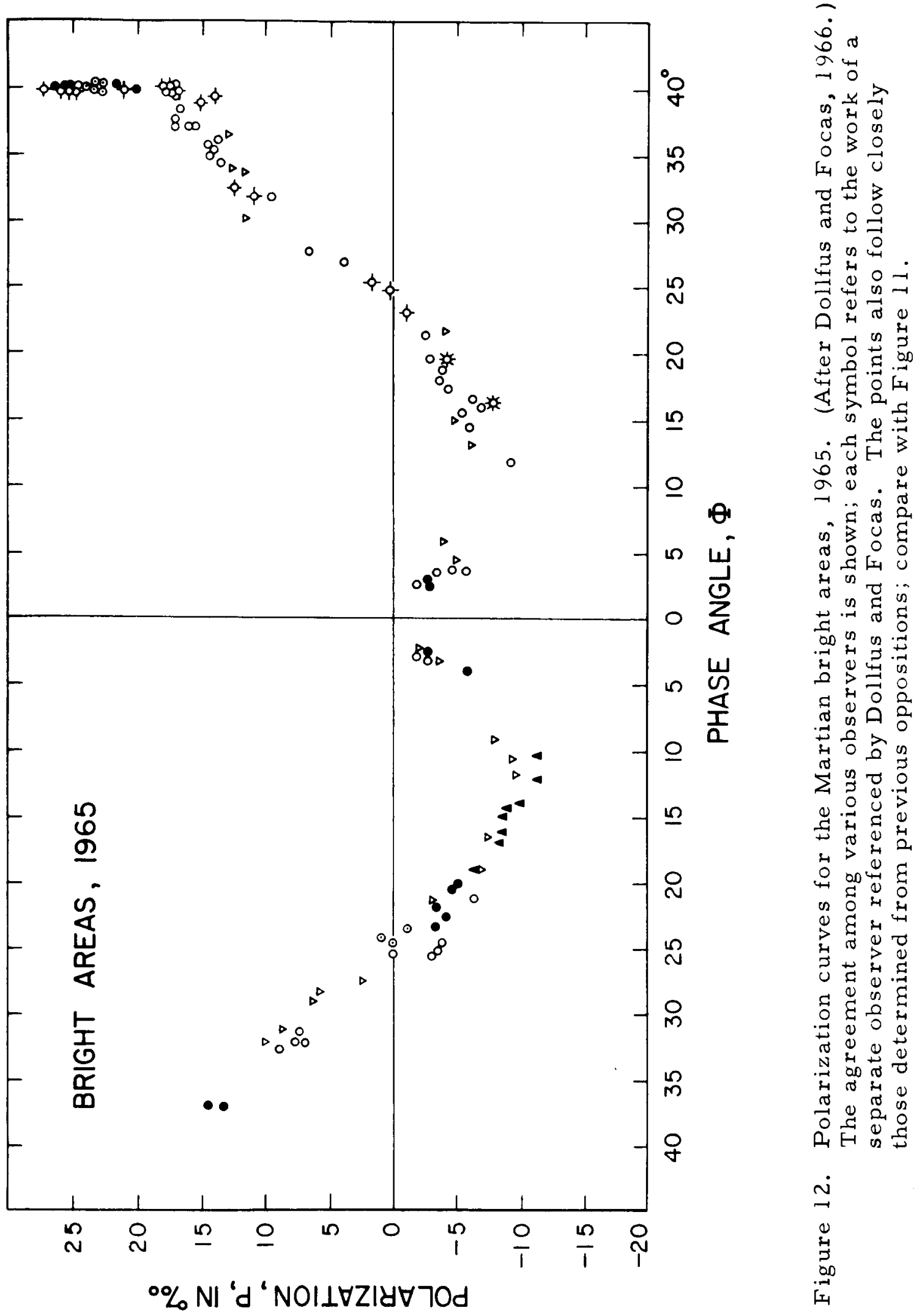


Dollfus (1957a) supposed that the aerosol particle dimensions were small compared with the wavelength of visible light, and that the particles would Rayleigh scatter as molecules; he corrected for total atmospheric polari zation accordingly. These suppositions were challenged by Rea and O'Leary (1965), who showed that correcting for aerosol particles with dimensions comparable to a wavelength leads to a vastly different polarization curve for the surface material. The aerosols themselves can show a strong negative branch for $\Phi<30^{\circ}$ and a strong positive branch for $\Phi>30^{\circ}$, leading to surface materials with residual polarization curves very different from those indicated by observations of aerosols and surface materials together.

More recent evidence makes the argument of Rea and O'Leary rather untenable. The $85-\mathrm{mb}$ pressure estimates were based on comparatively few observations, and the various methods employed by Dollfus showed as much as a factor of 2 variation in the inferred surface pressures. The old estimates also suffered from poorly known surface properties. For example, Dollfus assumed the polarization curve of the surface would be wavelength independent. His new strategy is to find such properties as wavelength dependence from the behavior of a sample of pulverized limonite that matches the $P-\Phi$ curve at $6100 \AA$. This necessarily introduces some circularity into the argument if limonite is to be deduced from the residual polarization after corrections for atmospheric polarization have been made; but, as we show below, the argument on surface composition nevertheless remains persuasive. Selecting the best data through the 1965 opposition, Dollfus has now lowered his original pressure estimate by a factor of about 3 (Dollfus and Focas, 1966). Pollack (1967) has recently made use of these data to obtain a more exact polarimetric estimate of surface pressure; he finds that for an atmosphere composed of $50 \% \mathrm{CO}_{2}$ and $50 \% \mathrm{~N}_{2}$, the surface pressure near the center of a bright area is about $19 \mathrm{mb}$. There is only a weak dependence on the as sumed $\mathrm{CO}_{2}$ abundance. This figure is compatible with other modern estimates of surface pressure if the bright areas measured are significantly depressed relative to the dark areas. There are several other lines of evidence indicating that such is indeed the case (Sagan and Pollack, 1967b; Sagan, Pollack, and Goldstein, 1967), and that the differences 
between spectrometric and occultation pressures can be understood on the same grounds (Sagan and Pollack, 1967b).

We now briefly consider the logic behind the new analysis of the polarization data. Both the polarization, $P$, and the brightness, B, can be measured as functions of $\lambda$, of $\Phi$, and of arcos $\mu$, the planetocentric angle from the center of the disk. At some sufficiently long wavelength the effect of atmospheric Rayleigh scattering and of aerosols on both $\mathrm{P}$ and $\mathrm{B}$ will be small. At $\lambda=6100 \AA$, there is very little obscuration of surface detail or time-dependent patchiness seen; and the pressures required for substantial Rayleigh scattering contributions at this wavelength can be excluded on other grounds. Dollfus and Focas measure $P(\Phi, 6100 \AA)$ and match these observations to the polarization curves of laboratory samples, especially limonite, which matches very well; $P(\mu, 6100 \AA)$ is then determined, both for Mars and for the successful laboratory samples. The difference between these results gives the atmospheric brightness, $B_{a}(6100 \AA)$. However, the scatter in the data points (Dollfus and Focas, Figure 29) is not small, possibly owing in part to limb clouds. Assuming that $\mathrm{B}_{\mathrm{a}}(6100 \AA)$ arises purely from Rayleighscattering, a value of the surface pressure is then derived. Note that this is a combined polarimetric and photometric method. It is of some interest to show that the contribution of the atmosphere to the total brightness is small. From Dollfus (1957a),

$$
P_{a}(\Phi, \mu, \lambda)=\frac{B_{a}(\Phi=0, \mu=1, \lambda)}{B_{s}(\Phi, \mu, \lambda)} \frac{\sin ^{2} \Phi}{2 \mu} .
$$

The observations then give an atmospheric brightness at the center of the disk, $\Phi=0^{\circ}$, and $\lambda=6100 \AA$, of $1 \%$ that of a bright area at the same $\Phi, \mu$, and $\lambda$.

The results for pressure at $6100 \AA$ A can then be checked by observations at a smaller wavelength, where the atmospheric contribution will be more pronounced. The surface contribution to $P(\Phi, \mu, \lambda)$ is taken as known, from laboratory measurements at the new wavelength on samples that 
successfully match the $6100 \AA$ data; $B(\Phi, \mu, \lambda)$ for the surface is taken directly from the observations. Dollfus and Focas choose $4700 \AA$ for the new wavelength. One might elect to measure $P(\mu, 4700 \AA)$, but the scatter at this wavelength, owing to seeing effects and limb clouds, will be very large; or to measure $B(4700 \AA)$, but the effect of the atmosphere on the total brightness will still be small and very difficult to measure. Dollfus and Focas instead measure $P(\Phi, 4700 \AA)$, because, at such properly chosen phase angles as $\Phi=40^{\circ}, \mathrm{P}_{\mathrm{a}} \sim \mathrm{P}_{\mathrm{S}}$ and the atmospheric influence will be easily measurable. This again yields $B_{a}(4700 \AA)$, which is found to differ from $\mathrm{B}_{\mathrm{a}}(6100 \AA)$ according to the Rayleigh $\lambda^{-4}$ law - implying that the new measurements are reasonably accurate and that Rayleigh scattering predominates.

This argument can be tested at many other wavelengths. When the atmospheric brightness, $B_{a}$, is much less than the surface brightness, $B_{s}$ (the situation for all wavelengths under consideration here), then the total polarization is given by

$$
P(\Phi, \lambda)=P_{s}(\Phi, \lambda)+\frac{\sin ^{2} \Phi}{1+\cos ^{2} \Phi} \frac{B_{a}(\Phi, \lambda)}{B_{s}(\Phi, \lambda)}
$$

(Dollfus, 1957a). If $\Delta P(\Phi, \lambda)$ is the difference between the observed planetary and the successful laboratory sample polarizations, then

$$
\Delta \mathrm{P}(\Phi, \lambda) \mathrm{B}_{\mathrm{s}}(\Phi, \lambda)=\frac{\sin ^{2} \Phi}{1+\cos ^{2} \Phi} \mathrm{B}_{\mathrm{a}}(\Phi, \lambda) \propto \lambda^{-4} .
$$

In Figure 13 is plotted $\Delta P(\Phi, \lambda) B_{s}(\Phi, \lambda)$ versus $\lambda$ for $\Phi=40^{\circ}$. The data points represented by rectangles are from Dollfus and Focas (1966), and the curve shows the $\lambda^{-4}$ dependence expected from equation (12). The areas of the rectangles indicate the internal scatter in the data set. The curve has been normalized to agree with the data at $6300 \AA$. The necessary values for $B_{s}(\Phi, \lambda)$ were obtained from the photometric work of Younkin and of Dollfus, discussed in an earlier section. We see that, within the probable errors of measurement, the observation's agree with the Rayleigh scattering curve. 
Finally, we discuss one other serious inconsistency with the suggestion put forth by Rea and O'Leary. The polarization curves obtained by Dollfus and Focas (1966) at $5000 \AA, 5300 \AA, 6300 \AA, 8300 \AA, 9500 \AA$, and $10,500 \AA$ all show exceedingly similar negative branches. A very similar result was obtained for laboratory limonite samples that matched $P(\Phi, 6300 \AA)$. But such behavior is distinctly unexpected for the Rea-O'Leary aerosols: The relative contribution of the atmosphere to the net polarization varies as $\mathrm{P}_{a}(\Phi, \lambda) \mathrm{B}_{\mathrm{a}}(\Phi, \lambda) / \mathrm{P}_{\mathrm{s}}(\Phi, \lambda) \mathrm{B}_{\mathrm{s}}(\Phi, \lambda)$. For particle dimensions comparable to a wavelength, a small change in $\lambda(6300 \AA$ to $5000 \AA$, say) will not change $\mathrm{P}_{\mathrm{a}}(\Phi, \lambda)$ or $\mathrm{B}_{\mathrm{a}}(\Phi, \lambda)$ appreciably. We have already mentioned that $\mathrm{P}_{\mathrm{s}}(\Phi, \lambda)$ varies only slightly over this wavelength range. But $B_{S}(\Phi, \lambda)$ is observed to decrease by about a factor of 2 between $6300 \AA$ and $5000 \AA$, a fact connected of course with the red coloration of the planet. Thus, if the atmosphere is significantly influencing the amplitude of the negative branch, the branch should show a marked change between $6300 \AA$ and $5000 \AA$, contrary to observation. It thus seems safe to reject the arguments of Rea and O'Leary that an atmospheric aerosol composed of very small particles (in order to influence the negative branch) is playing a major role in determining the Martian polarization curve.

In summary, the new polarization results lead to a surface pressure compatible with other estimates. The atmospheric scattering approximately follows a Rayleigh scattering law, a result in agreement with Evans' (1965) data in the rocket ultraviolet. Not only do the polarization data not require a substantial aerosol contribution, they are inconsistent with such a contribution.

\section{3 Physics of the Polarization Curves}

In this section we discuss the mechanisms producing polarization upon the scattering of initially unpolarized light off a surface, and the physical parameters influencing the polarization. We note from Figures 10 and 11 that at small phase angles the Martian polarization curves a re negative, while at larger phase angles, $P$ becomes positive. In late $r$ discussion, we will refer 
to several parameters characterizing these curves. The only extremum on the curves occurs at the negative maximum; the polarization and phase angle of this point we call $P_{1}=\mathrm{P}[(\mathrm{dP} / \mathrm{d} \Phi)=0]$ and $\Phi_{1}=\Phi[(\mathrm{dP} / \mathrm{d} \Phi)=0]$, respectively. The polarization zero not at the origin is at phase angle $\Phi_{2}=\Phi(P=0, \Phi \neq 0)$. Finally, we will be concerned with the slope of the positive branch at large phase angles, $S \equiv \mathrm{dP} / \mathrm{d} \Phi\left(\Phi>\Phi_{2}\right)$. These four parameters are illustrated in Figure 14.

The mechanism producing the positive branch is readily understood. It is simply the result of reflection events, which according to Fresnel's laws always lead to positive polarization with the present sign convention: Polarization is defined with respect to the plane of vision - here the plane Sun-Mars-Earth - and is positive when the electric vector component normal to this plane is larger than the component in the plane. We consider a powder composed of grains in a variety of orientations. Those grains with a local normal in the plane of vision, and inclined to the planetary surface by half the phase angle, will reflect light to the Earth with the polarization expected when the angle of incidence equals $\Phi / 2$. The sum of the angles of incidence and reflection will equal the phase angle. The polarization from such single Fresnel scattering will increase from $P=0$ at $\Phi=0^{\circ}$ to a maximum $\mathrm{P}$ at the maximum Martian phase angle currently accessible, $\Phi \simeq 45^{\circ}$. From the Fresnel laws, multiple reflections depolarize, even for nonrandom geometry, as with stacked plates. With random geometry of scatterers, depolarization is enhanced through the los of any initial $\mathrm{E}$-vector orientation. For both single and multiple scattering the positive branch is weakest at small phase angles, and it is therefore here that sources of negative polarization are most likely to dominate.

The single-scattering albedo, $\tilde{\omega}_{0}$, of the grain measures the fraction of the incident radiation that is reflected off the grain, diffracted around it, or transmitted through it; it is clearly to be distinguished from the Fresnel reflectivity. The absorption within the particle is measured by $1-\tilde{\omega}_{0^{\circ}}$ As $\tilde{\omega}_{0}$ becomes small, reflection events make up an increasing fraction of the emergent intensity, and the positive polarization is maximized. For a fixed 


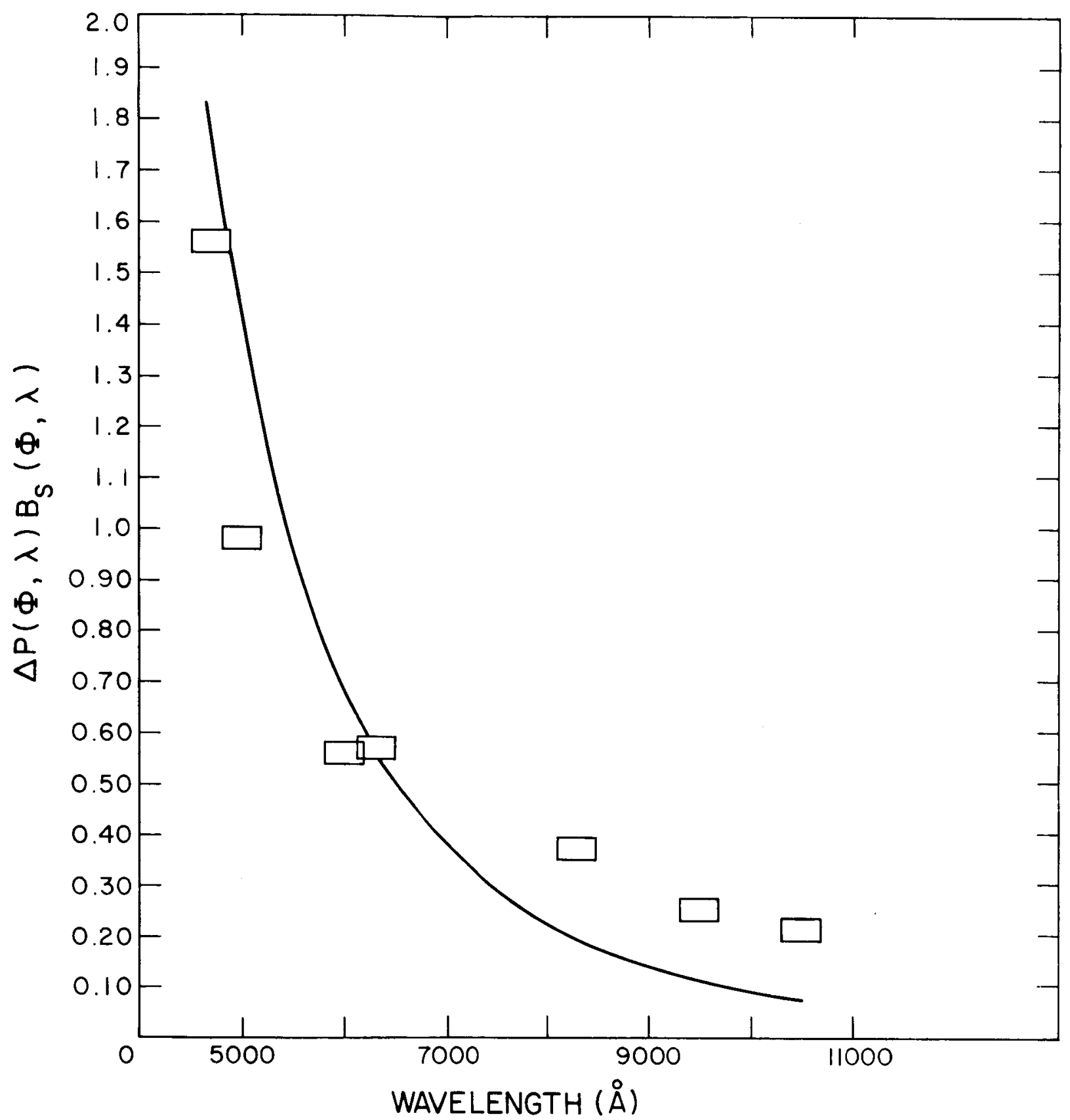

Figure 13. Comparison of the observed (open rectangles, after Dollfus and Focas, 1966) and theoretical values of $\Delta P(\Phi, \lambda) B_{S}(\Phi, \lambda)$. Both sets of data are for $\Phi=40^{\circ}$. The theoretical curve given by the solid line follows the Rayleigh $\lambda^{-4}$ dependence. 


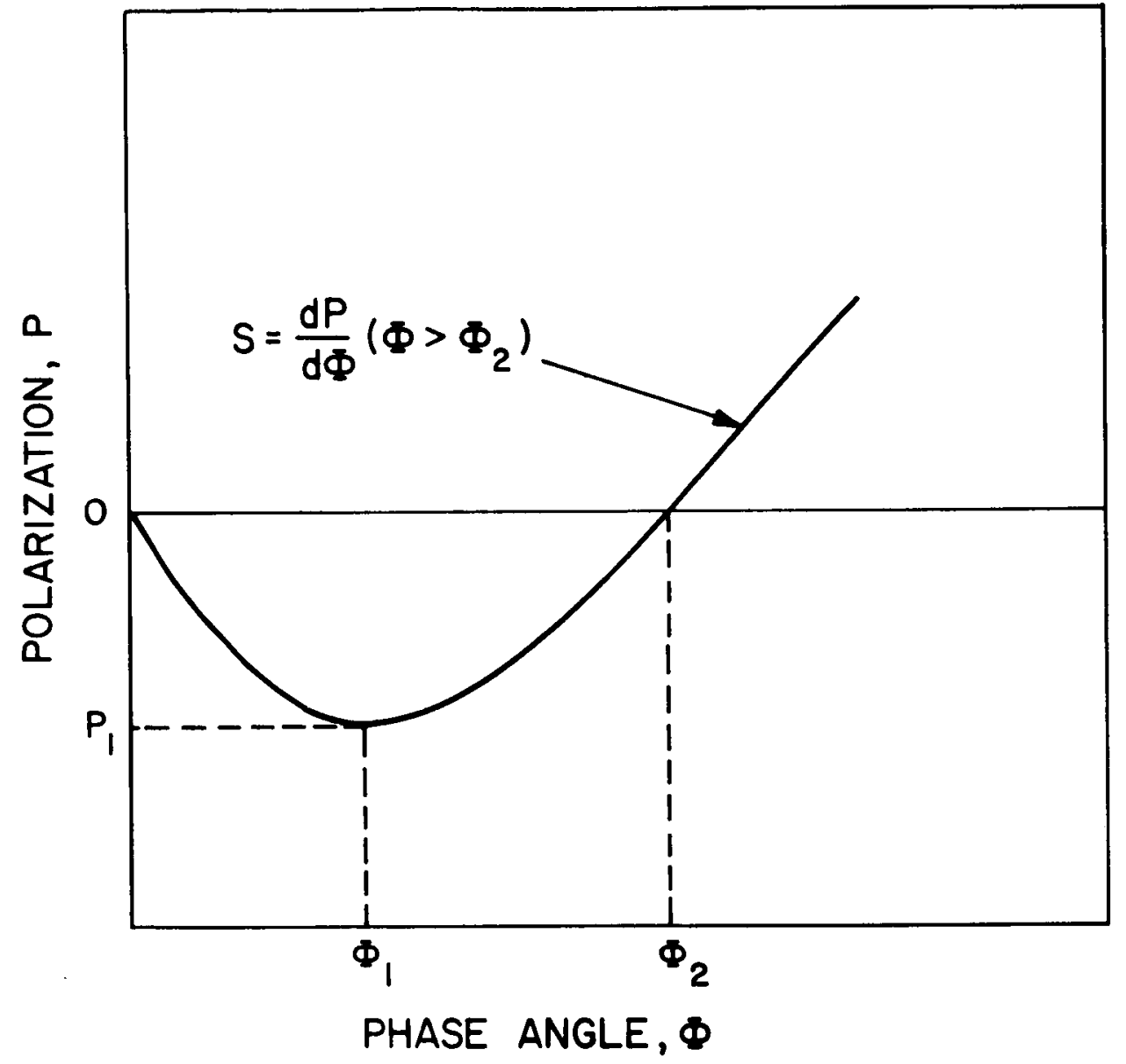

Figure 14. Schematic representation of fundamental parameters for characterizing polarization curves. 
$\widetilde{\omega}_{0}>0$, the polarization increases with increasing values of $n_{r}$, the real part of the index of refraction, because reflected radiation constitutes a larger fraction of the emergent flux. Thus, the positive branch is determined by two physical parameters, $\widetilde{\omega}_{0}$ and $n_{r}$.

Consider now a source, as yet unspecified, of negative polarization. For $\tilde{\omega}_{0}>0$, we increase $n_{r}$. The positive contribution then increases; $\Phi_{1}$ and $\Phi_{2}$ will occur at smaller phase angles; $\left|P_{1}\right|$ will decrease; and $\mathrm{d} P / \mathrm{d} \Phi\left(\Phi>\Phi_{2}\right)$ and $\mathrm{P}\left(\Phi>\Phi_{2}\right)$ both increase. These predictions we make here are borne out by Dollfus' (1957a, Figures 51 and 52) laboratory studies, in which the polarization curves of zircon $\left(n_{r} \simeq 1.95\right)$ and rutile $\left(n_{r} \simeq 2.75\right)$ are compared, as are the curves of limonite $\left(n_{r} \simeq 2.25\right)$ and hematite $\left(\mathrm{n}_{\mathrm{r}} \simeq 3.0\right)$.

The origin of the negative branch is not yet completely understood. However, it must somehow be the result of multiple scattering since a single Fresnel reflection leads only to positive polarization. This is vividly brought out in an experiment performed by Dollfus (1956): a powdered sample, on the ground, generates a negative branch; it does not do so when suspended in air so that only single scattering events are observed.

Hopfield (1966) has proposed that the origin of the negative branch lies in the diffraction of light around one particle and the scattering of the diffracted light back to space by another particle. The polarization generated in this way has the sign, magnitude, and $\Phi$-domain of the observed negative branch for opaque powders. Unfortunately, a number of unrealistic assumptions - such as extremely high electrical conductivities for the diffracting particles - are made in the derivation, and further work on this theory is needed. It does, however, make a number of additional predictions that are confirmed by experiment. The value of $|P|$ is predicted to increase monotonically as diffraction becomes more important; i. e., as a/ $\lambda$ decreases. Completely opaque iron filings show a pronounced enhancement in the negative branch as a/ $\lambda$ decreases (Dollfus, 1956, Figure 12). Since, for all sizes 
considered, the iron particles were completely opaque, the positive branch should be affected only to the extent that the source of negative polarization influences it. This prediction is confirmed by the observations: the various polarization curves asymptotically approach the same value of $\mathrm{dP} / \mathrm{d} \Phi$ at large phase angles, where the positive component dominates. The diffraction theory also indicates that the negative branch is enhanced if the grains are characterized by a fairly open structure; otherwise, the grains will not be sufficiently far apart for the diffracted component to reach the secondary scatterer with the appropriate geometry. In the laboratory, the negative branch is enhanced when the sample is stirred; when the sample is smoothed and its surface flattened, $|P|$ declines (Dollfus, 1956, Figures 13 and 14). In this experiment also, the positive contribution is unaffected by the grain packing; there is an asymptotic approach to the same $\mathrm{dP} / \mathrm{d} \Phi$ at large $\Phi$.

It is conceivable that negative polarization can also be produced by geometric optics. Öhman (1955) has pointed out that a retrodirective reflector viewed at phase angles away from $0^{\circ}$ leads to a negative polarization. However, the double reflections (with intensities $\propto \mathrm{R}^{2}$ ) yield much lower intensities than single scattering events, and there is some question of the geophysical abundance of retrodirective reflectors.

However, let us consider a beam of unpolarized light that undergoes $\underline{t}$ transmissions and $\underline{\mathbf{r}}$ reflections; $\underline{\mathrm{t}}$ and $\underline{\mathbf{r}}$ will be related by the geometry of the scattering. Since reflections lead to $P>0$, transmissions must produce $\mathrm{P}<0$. If we define the Fresnel reflectivities for the electric vector perpendicular and parallel to the plane of vision by $R_{\perp}$ and $R_{\|}$, respectively, the net polarization is then

$$
P \propto\left[R_{\perp}^{r}\left(1-R_{\perp}\right)^{t}-R_{11}^{r}\left(1-R_{11}\right)^{t}\right],
$$

approximately; the sign of $\mathrm{P}$ will be given by the sign of the quantity in square brackets in (13) [cf. equations (13) and (10)]. From the Fresnel equations, $R_{\perp}>R_{\| l}$ except when the angle of incidence is $0^{\circ}$ or $90^{\circ}$, in which case 
$\mathrm{R}_{\perp}=\mathrm{R}_{\|}$. For ordinary values of $\mathrm{n}_{\mathrm{r}}, \mathrm{R}_{\perp}<1 / 2$ and $\mathrm{R}_{\|}<1 / 2$, in general. Negative polarization will occur when $\left[\mathrm{R}_{\perp} / \mathrm{R}_{\|}\right]^{\mathrm{r}}\left[\left(1-\mathrm{R}_{\perp}\right) /\left(1-\mathrm{R}_{\|}\right)\right]^{\mathrm{t}}<1$. Since $\left[R_{\perp} / R_{\|}\right] \geq 1$, and $\left[\left(1-R_{\perp}\right) /\left(1-R_{\|}\right)\right] \leq 1$, significant negative polarization by this mechanism requires $t / r>1$. As $n_{r}$ increases, both $R_{\perp}$ and $R_{\|}$ increase, $\left[\left(1-R_{\perp}\right) /\left(1-R_{\|}\right)\right]$becomes smaller, and a smaller value of $t / r$ is required to achieve a given negative polarization. But also, as $n_{r}$ increases, the probability of achieving t transmissions $\left[\sim(l-R)^{t}\right]$ decreases. In a range of computations on Fresnel polarization of light scattered off stacked plates, with a wide range of values of $n_{r}, k, \Phi$, and the albedo of the underlying surface, no cases were found where the net polarization was negative for small phase angles, although individual refracted rays did sometimes show $\mathrm{P}<0$ (Sagan and Cruz-Gonzales, 1967, unpublished calculations).

Both the diffractive and refractive contributions to negative polarization decline as $\tilde{\omega}_{0} \rightarrow 1$; a larger fraction of the returned radiation then originates from multiple scattering, which randomizes any preferred $\mathrm{E}_{\mathrm{m}}$-vector orientation and therefore depolarizes. Transparent materials generally exhibit very weak negative branches. If $\tilde{\omega}_{0}$ is sufficiently small, exp(-kta) will be much less than unity, and refractive contributions to $\mathrm{P}<0$ at small phase angles will again be unlikely; this does not apply to the diffractive contribution. For the refractive contribution to be at all effective, multiple scattering must be reduced, implying high values of $n_{r}$ and small values of $t$.

The negative contribution thus depends on $a / \lambda, \widetilde{\omega}_{0}$, the degree of openness of structure, and possibly on $n_{r}$. The positive contribution is independent of $a / \lambda$ and the openness of structure, as we have seen. Some of the foregoing points are illustrated in Figure 15, polarization curves for limonite as a function of $\lambda$ (Dollfus and Focas, 1966). The positive branch decreases in amplitude toward larger wavelengths. This is because $\widetilde{\omega}_{0}$ is increasing with increasing $\lambda$, enhancing the contribution from multiple scattering. A similar effect occurs for the negative branch, but it is offset (1) by the increasing contribution from diffraction (a/ $\lambda$ decreasing toward large $r \lambda$ ), and (2) possibly by an increased contribution from refraction, as discussed above. 


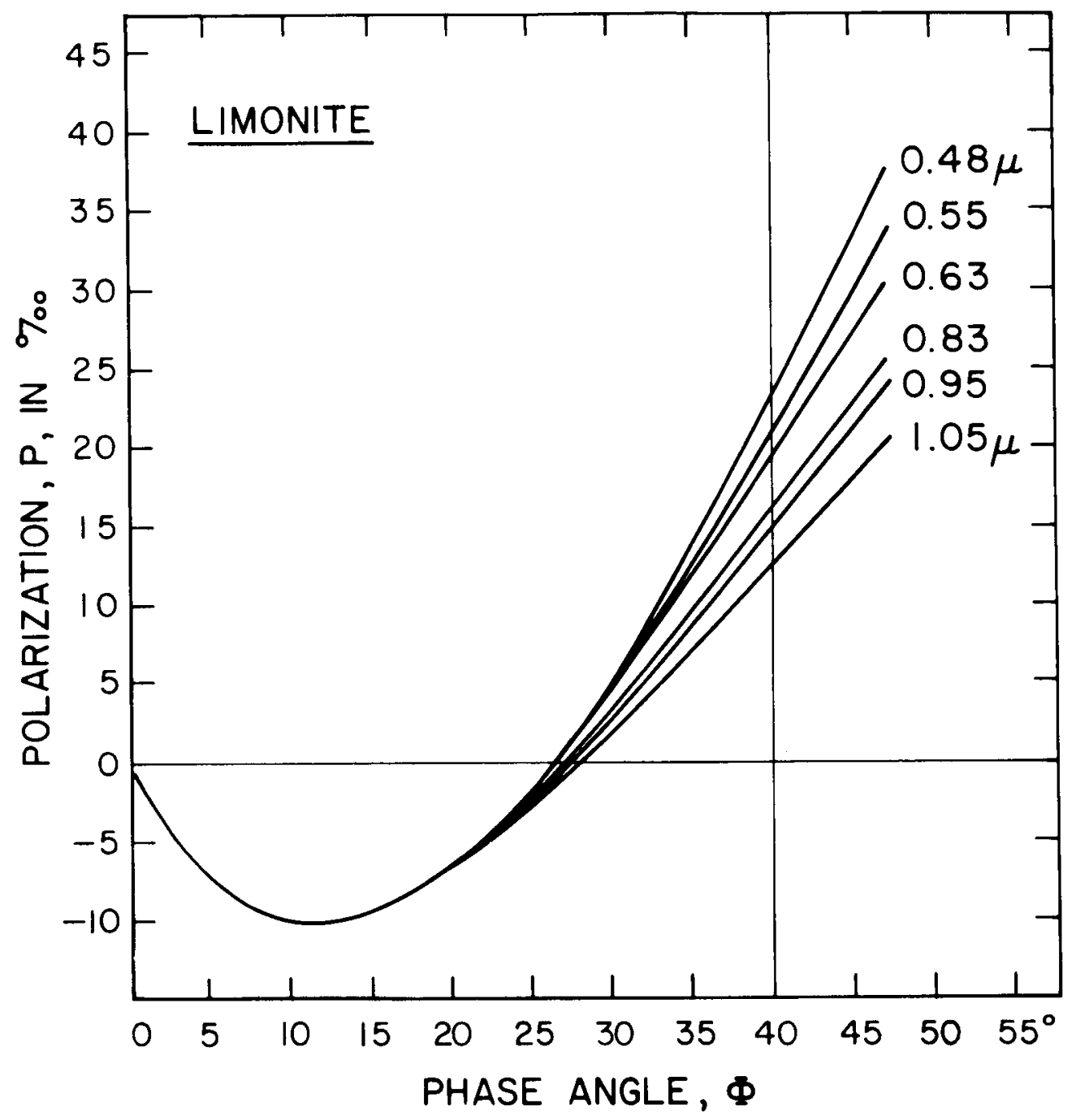

Figure 15. Wavelength dependence of the polarization curve of a sample of pulverized limonite that matches the Martian observations at a wavelength of $0.63 \mu$. After Dollfus and Focas (1966). 
3. 4 Implications of the Martian Polarization Curves

We now consider the interpretation of the polarization curves for the Martian bright and dark areas in terms of our understanding of the physics of such curves and utilizing a few of the conclusions we have drawn from the photometric observations. We have already seen that the presence of a prominent negative branch for both bright and dark areas implies pronounced multiple scattering; the Martian surface is therefore covered either with extremely porous rocks or with a fine powder, in agreement with the photometric results. The dark areas seen are known to be a patchwork of brighter and darker features; but the negative branch is, in fact, more pronounced in average dark areas than in average bright areas. Therefore, the negative branch of the dark areas is not simply due to the brighter features present within them.

\section{4. 1 Analytic strategy}

Our focus in the discussion immediately below is to reproduce the observed Martian polarization curves with terrestrial laboratory samples. We have seen that four parameters $-\mathrm{n}_{\mathrm{r}}, \widetilde{\omega}_{0}, \mathrm{a}$, and $\mathrm{y}$, the degree of openness of structure - specify the polarization curve at a given wavelength. By employing the deductions in our discussion of the photometry, we can fix the value of one of these parameters - the single-scattering albedo - quite accurately. We require each laboratory sample to have the same reflectivity as actually observed for the Martian bright areas. Once $\widetilde{\omega}_{0}$ is so fixed, the positive polarization will be controlled by $\mathrm{n}_{r}$, and the negative polarization by $\mathrm{n}_{\mathrm{r}}, \mathrm{a}$, and $\mathrm{y}$. The four empirical polarization parameters, $\Phi_{1}, \Phi_{2}, \mathrm{P}_{1}$, and $\mathrm{S} \equiv \mathrm{dP} / \mathrm{d} \Phi\left(\Phi>\Phi_{2}\right)$ are functions of positive and negative contributions to the polarization curve. The superpositions of these contributions determine the positive and negative branches of the polarization curve. The positive contribution is a function of $\widetilde{\omega}_{0}$ and $n_{r}$ alone, or - with $\widetilde{\omega}_{0}$ specified photometrically - a function of $\mathrm{n}_{\mathrm{r}}$ alone. Therefore, in principle, a meas urement of $S$ at sufficiently large $\Phi$ should determine $n_{r}$. However, $S$ is 
more difficult to measure than $\Phi_{1}, \Phi_{2}$, or $P_{1}$ (see below), and it is not clear that for Mars we can measure at sufficiently large $\Phi$ to be confident of no significant influence on $S$ by the negative contribution. Therefore, we choose to consider also the parameters of the negative branch, which are affected by both positive and negative contributions. If, for a given sample, we adjust $y$ to give the correct value of $P_{1}$ (and this will not always be possible) then $\Phi_{1}, \Phi_{2}, S$ and $P\left(\Phi>\Phi_{2}\right)$ will all be determined by the positive contribution, and thus will depend only on $n_{r}$. We have seen that, for the case of interest $\left(\tilde{\omega}_{0}>0\right)$, increasing $n_{r}$ results in smaller $\Phi_{1}$ and $\Phi_{2}$, and larger $S$ and $P\left(\Phi>\Phi_{2}\right)$. Since the reflectivity is quite wellknown, $n_{r}$ will be well determined if we find a sample that matches both the photometric and the polarimetric data. With $n_{r}$ thus specified, and a determined photometrically for a given sample, an estimate of $y$ for the same sample can be extracted from the negative polarization. We note again that the value of $n_{r}$ deduced for Mars will be the same for all successful samples; this is not the case for $y$. Thus the photometry and polarimetry together may permit us to determine $n_{r}, \tilde{\omega}_{0}, a$, and $y$ together.

\section{4.2 Determining $\mathrm{n}_{\mathrm{r}}$ and $\mathrm{y}$}

Dollfus (1957a) has, in effect, followed this strategy. In his earlier work, he took the four years of polarimetry represented in Figure 11 and obtained a mean polarization curve for the Martian bright areas. He then corrected for the effect of the atmosphere on the polarization curves, using $\mathrm{B}_{\mathrm{a}} / \mathrm{B}_{\mathrm{s}}=2.8 \times 10^{-2}$ at $\lambda=6100 \AA$, a value almost three times larger than his more recent determinations (Dollfus and Focas, 1966). The effect of the original atmospheric correction was to leave $P_{1}$ and $\Phi_{1}$ essentially unchanged, to increase $\Phi_{2}$ by $3^{\circ}$, and to decrease $S$ by some $20 \%$. This corrected curve then agreed very well with a laboratory sample of pulverized limonite that showed the same value of $\tilde{\omega}_{0}$ as the bright areas. The sample was stirred with a rod to create an open structure (increased y).

The new and lower value of the atmospheric correction implies that the particular limonite sample that was previously taken to match the Martian polarization curves will no longer quite match them. However, the resulting 
discrepancy is quite small, perhaps within the errors of observation; in any case, as discussed below, a more nearly exact agreement can probably be secured with other samples of limonite. By contrast (cf. Figure 10), hematite does not fit the data at all: $\Phi_{1}$ (Mars) $=\Phi_{1}$ (limonite) $=12^{\circ}$; $\Phi_{1}$ (hematite $)=5^{\circ} ; \Phi_{2}($ Mars $)=27^{\circ} ; \Phi_{2}($ limonite $)=29^{\circ} ; \Phi_{2}$ (hematite) $=18^{\circ} ;$ in addition, $\mathrm{dP} / \mathrm{d} \Phi\left(\Phi>\Phi_{2}\right)$ is much too large for hematite. All these differences arise from the fact that $\mathrm{n}_{\mathrm{r}}$ (hematite) $\approx 3.0$ is significantly greater than $n_{r}$ (limonite) $\simeq 2.25$; they indicate how sensitively the Martian polarization curves depend upon the real part of the refractive index. In going from hematite to goethite, there is a change in crystal structure, which accounts for most of the change in $\mathrm{n}_{\mathbf{r}}$. In going from goethite to limonite there is no change in crystal structure, and, accordingly, a very small change in $n_{r}$ - arising in part from the addition of bound and adsorbed water.

If the limonite sample employed by Dollfus (1957a, p. 109) had a slightly higher visible reflectivity than the Martian bright areas, then the sample reflectivity should be decreased, $\tilde{\omega}_{0}$ decreased, and the positive contribution to the polarization curve enhanced for fixed $n_{r}$. If in addition we keep $P$ the same by fixing y for a given sample, then all the other properties of the polarization curve will change in the desired direction.

A second possible method of improving the (already good) agreement is to raise slightly $n_{\mathbf{r}}$ (i. e., by several hundredths). Since limonite has variable quantities of other minerals and of adsorbed water while still retaining goethite as its principal constituent, $n_{r}$ is expected to vary somewhat from sample to sample. A comparison of $n_{r}$ (limonite) given by Forsythe (1954) with $\mathrm{n}_{\mathrm{r}}$ (pure goethite), however, shows a difference of only 0. 05. Thus, as long as goethite is a principal constituent, $n_{r}$ for different limonite samples should not vary much from sample to sample. In summary, the properly corrected polarization curve for the Martian bright areas is in satisfactory agreement with that of limonite. In particular, ${ }_{r}\left(\sigma^{x}, b\right)$ is very close to $n_{r}$ (limonite), which, moreover, seems to have a well-defined value among naturally occuring samples. 
On the other hand, if goethite is not a principal constituent of the Martian bright areas, it is highly unlikely that $n_{r}\left(\sigma^{4}\right.$, b) would fall in the limonite range. A similar conclusion holds for mixtures of hematite and minerals that are not largely iron oxides. Similarly, a turgite sample, consisting primarily of $2 \mathrm{Fe}_{2} \mathrm{O}_{3} \cdot \mathrm{H}_{2} \mathrm{O}$, a ferric oxide with hydration state intermediate between those of hematite and goethite, is apparently ruled out as a major constituent because $n_{r}$ (turgite $) \simeq 2.52$. Finally, the frequently made suggestion that the ferric oxides on Mars may be present only as a thin patina of "desert varnish" can apparently also be excluded by these considerations. Were we to coat a quartz core with goethite, the effective value of $n_{r}$ would exceed that for a pure sample of goethite, because of reflection at both the air-goethite and the goethite-quartz interfaces. From Fresnel's laws at normal incidence, we estimate the effective value of the real part of the refractive index for this hybrid particle to be $n_{r} \simeq 2.57$, significantly larger than the values necessary to explain Martian polarimetry. We again conclude that goethite is a principal constituent of the Martian bright areas.

To achieve the correct values for the parameters that characterize the negative branch, while approximately fitting the rest of the polarization curve, the limonite sample was given a very open structure. This result is consistent with the requirements set by the radar data on the assumption that the bright areas are primarily limonite (Sagan and Pollack, 1967c), and is also consistent with the frequent observations of bright-area particles lifted by winds and subsequently settling out.

\section{4. 3 Comparison of bright-and dark-area polarimetry}

We next consider the polarization curve of the dark areas, and attempt to pursue the hypothesis that the dark areas are also composed in significant part of pulverized limonite, but with larger particles in the dark than in the bright areas. Figure 16 shows polarization curves for both coarse-grained $\left(L_{1}\right)$ and fine-grained $\left(L_{2}\right)$ samples of limonite powder (Dollfus and Focas, 1966). The dashed Mars curves can be used to compare these curves. 


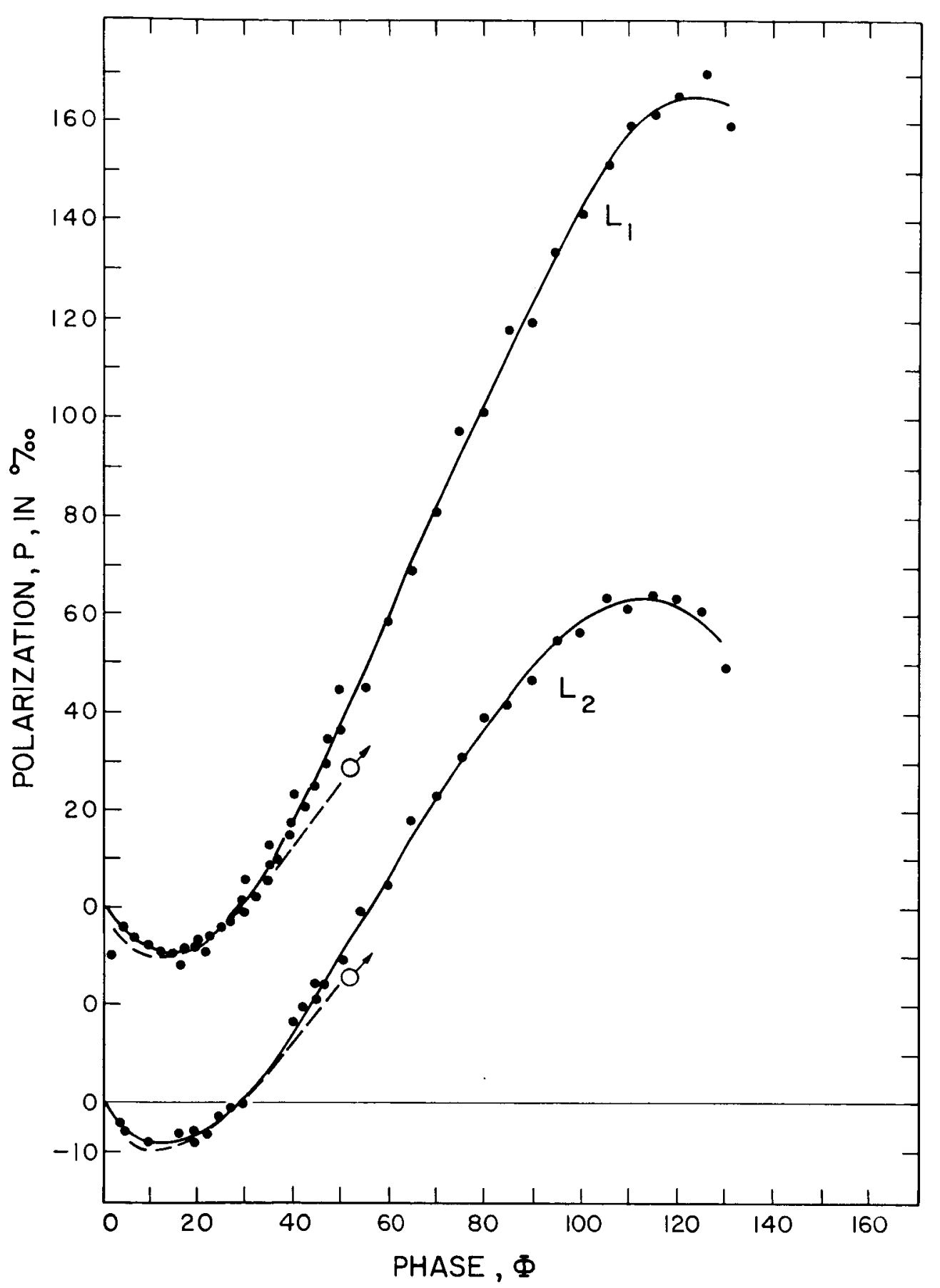

Figure 16. A comparison at $0.64 \mu$ of laboratory polarization curves of pulverized limonite for coarse grains (upper curve, $L_{l}$ ) and fine grains (lower curve, $L_{2}$ ). Shown for comparison are the corresponding curves for Mars given by the dashed lines. 
The limonite values of $P_{1}$ are in the Martian range. Both the negative and positive contributions to the net polarization - as judged by $P_{1}$ and $S-$ are enhanced for the coarse-grained sample. For the positive contribution, this is expected because of the consequent smaller $\widetilde{\omega_{0}}$. For the negative contribution, the smaller $\tilde{\omega}_{0}$ has evidently offset the larger value of $a / \lambda$ and the resulting decreased contribution from diffraction. We see that the effect of increasing a is to increase $\left|P_{1}\right|$ slightly, increase $\Phi_{2}$ very slightly, and increase S significantly. From Figure 10 we find that the polarization curve $\mathrm{d}^{\mathrm{a}}$ of the dark areas at the height of the seasonal darkening differs from the polarization curves, $b^{\alpha}$ and $b^{\beta}$, of the bright areas in just the same manner that the coarse-grained curve differs from the fine-grained curve. Curve $b^{\beta}$ refers to bright areas seen in the 1952 and 1954 oppositions near $0^{\circ}$ latitude; curve $b^{a}$ to bright areas seen in the 1948 and 1950 oppositions near $+20^{\circ}$ latitude. It is worthwhile to note also that curves $b^{a}$ and $b^{\beta}$ differ from one another in exactly the same sense as curve $d^{a}$ differs from them both. We have already inferred from the photometric results that the bright and dark areas may differ chiefly in the mean particle size; we now find supporting evidence from the polarimetry. As we discuss below, the corrections for atmospheric polarization will not affect these qualitative conclusions.

We now consider the comparison between bright-and dark-area polarimetry in more detail. We will restrict our attention to the four oppositions of 1948,1950, 1952, and 1954 because the data here represent a large number of observations made with the same telescope and the same polarimeter. The 1954 data were obtained primarily by Focas, who was trained by Dollfus in the use of Dollfus' polarimeter. The remaining data sets were obtained by Dollfus himself. We group the dark areas into three categories (cf. Dollfus, 1957a) according to latitude. Group 1 comprises bright and dark areas between latitudes $+60^{\circ}$ and $+15^{\circ}$, and includes the dark areas Panchaia, Boreosyrtis, Mare Acidalium, and Lacus Niliacus. Group 2 comprises areas between latitudes $+15^{\circ}$ and $-45^{\circ}$ and includes Syrtis Major, Margaritifer Sinus, Sinus Sabaeus, Mare Tyrrhenum, Mare Cimmerium, Mare Sirenum, Mare Erythraeum, and Iapigia. Group 3 comprises latitudes $+15^{\circ}$ to $-15^{\circ}$, and includes the first three members of 
Group 2. We further group according to year. Interval a refers to the 1948 and 1950 oppositions when Group 1 dark areas were substantially darkened in the Northern Hemisphere seasonal changes. Interval $\beta$ refers to the 1952 and 1954 oppositions, when there was little seasonal darkening in either hemisphere. With $b$ and $d$ standing, respectively, for bright and dark areas, such symbols as $d_{2}^{a}$ should be clear. We now construct smooth curves for various latitude groups, time intervals, and surface areas from Dollfus' (1957a) original data points. Figures 17 through 20 show the resulting curves. Table 4 summarizes the characteristic parameters of the polarization curves. Because the slope is not uniquely defined, we have arbitrarily standardized as the mean slope that between $\Phi_{2}$ and $\Phi=36^{\circ}$. The parameters $\Phi_{1}$ and $\Phi_{2}$ can be measured to $\simeq 1.5^{\circ}, \mathrm{P}_{1}$ to about $0.2 \%$; $\mathrm{dP} / \mathrm{d} \Phi\left(\Phi>\Phi_{2}\right)$ is considerably more uncertain, both because of the relative paucity of data points and because of the curvature of the positive branches. Absolute values are probably good to $20 \%$, and relative values are probably more reliable - e.g., we can assert with reasonable confidence that $S$ is smaller for $b^{\beta}$ than for $b^{a}$.

Consider now the effect of reasonable corrections for atmospheric polarization, given by equation (11). Subtracting these corrections from the curves of Figures 17 to 20 yields a corrected curve for the Martian surface. A value of $B_{a} / B_{s} \simeq 10^{-2}$ was used for bright areas at the center of the disk. Values of $B_{a} / B_{s}$ for the darkareas were derived with the aid of Focas' (1961) plot of brightness ratios of various bright areas to various dark areas, as a function of heliocentric longitude. Table 4 gives values of $P_{1}, \Phi_{1}, \Phi_{2}$, and $S$ for the curves so corrected. It is evident that the influence of this correction is small, both on absolute and on relative values. Accordingly, our conclusions are unlikely to be affected by the uncertainties in the corrections for atmospheric polarization.

We see that both bright and dark areas have about the same values of $\Phi_{1}, P_{1}$, and $\Phi_{2}$; but $S$ is greater for the dark areas. Figures 18 to 20 present comparisons between bright and dark areas. We see that dark-area polarization curves during the seasonal darkening differ from such curves away from the 

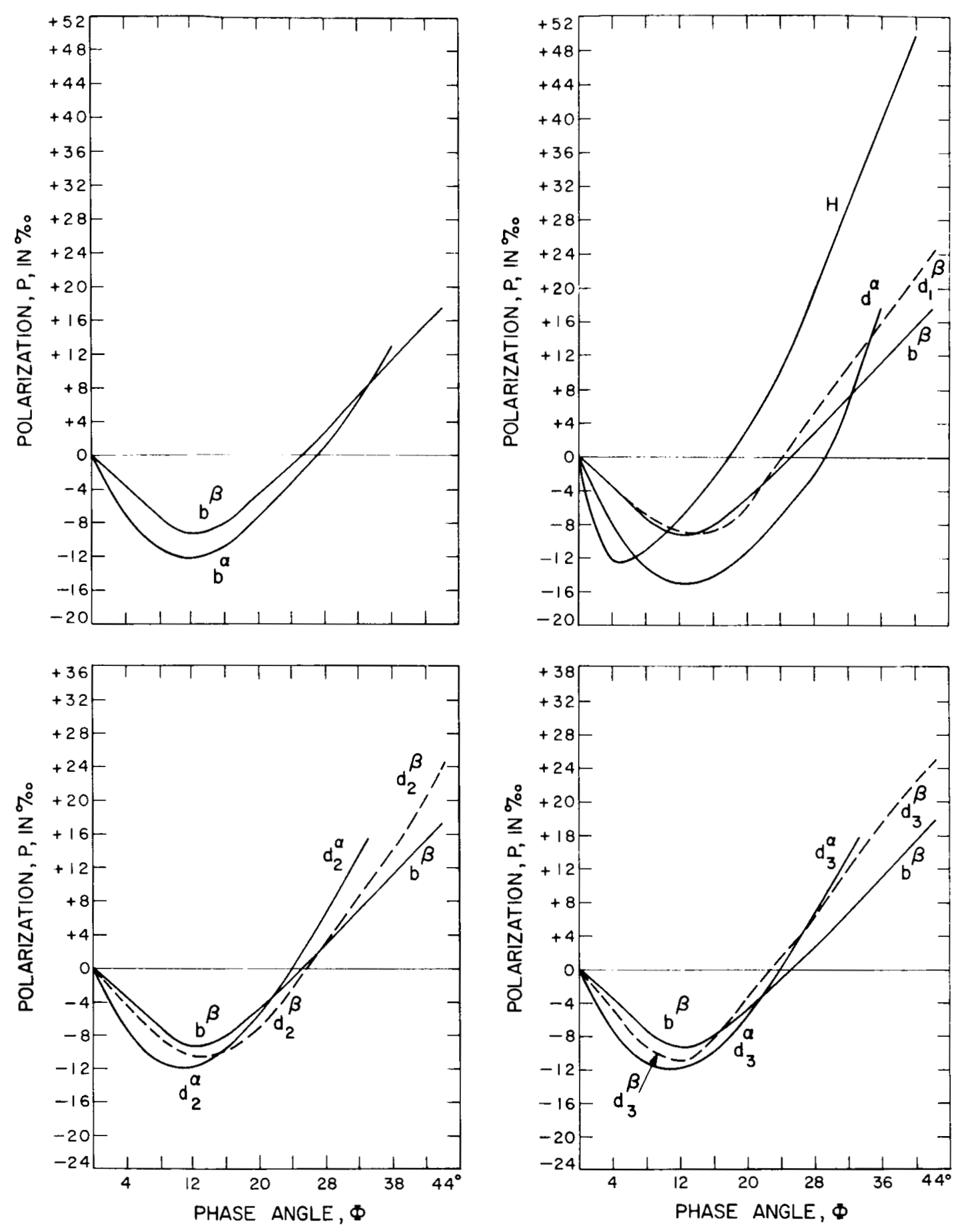

Figures 17-20. Polarization curves of selected bright and dark areas of Mars at various times during the 1948, 1950, 1952, and 1954 oppositions for several latitudes. See Table 4 for details. The bright areas are designated by $b$, the dark areas by $d$. The oppositions of 1948 and 1950 are indicated by superscript $a$, while the oppositions of 1952 and 1954 are indicated by superscript $\beta$. The subscripts 1,2 , and 3 refer to various latitude regions. 
Table 4. Parameters for polarization curves uncorrected and corrected for atmospheric polarization

\begin{tabular}{|c|c|c|c|c|c|c|c|c|}
\hline \multirow{3}{*}{$\begin{array}{l}\text { Region } \\
\text { code }\end{array}$} & \multicolumn{2}{|c|}{$\Phi_{1}$} & \multicolumn{2}{|c|}{$P_{1}$} & \multicolumn{2}{|c|}{$\Phi_{2}$} & \multicolumn{2}{|c|}{$\mathrm{dP} / \mathrm{d} \Phi\left(\Phi>\Phi_{2}\right)$} \\
\hline & \multicolumn{2}{|c|}{ Corrected } & \multicolumn{2}{|c|}{ Corrected } & \multicolumn{2}{|c|}{ Corrected } & \multicolumn{2}{|c|}{ Corrected } \\
\hline & No & Yes & No & Yes & No & Yes & No & Yes \\
\hline$b^{a}$ & $12^{\circ}$ & $12^{\circ}$ & $-12.3 \% 0$ & $-12.5 \%$ & $27.2^{\bullet}$ & $28.1^{\circ}$ & 1.48 & 1.42 \\
\hline$b^{\beta}$ & 12 & 12 & -9.3 & -9.5 & 25.2 & 26.3 & 1.22 & 1.18 \\
\hline$d_{1}^{a}$ & 13 & 13 & -15.0 & -15.5 & 29.4 & 30.5 & 2.86 & 2.76 \\
\hline$d_{2}^{a}$ & 11 & 11 & -12.0 & -12.4 & 24.0 & 25.3 & 1.78 & 1.65 \\
\hline$d_{3}^{a}$ & 11 & 11 & -12.0 & -12.4 & 24.0 & 25.3 & 1.78 & 1.65 \\
\hline$d_{1}^{\beta}$ & 14 & 14 & -9.0 & -9.7 & 24.4 & 26.0 & 1.72 & 1.59 \\
\hline$d_{2}^{\beta}$ & 13 & 13 & -10.5 & -10.9 & 26.2 & 27.5 & 1.61 & 1.48 \\
\hline$d_{3}^{\beta}$ & 12 & 12 & -11.0 & -11.4 & 22.8 & 24.1 & 1.67 & 1.54 \\
\hline $\mathrm{H}$ & & & -1 & & & & & \\
\hline
\end{tabular}


seasonal darkening in the same sense that they differ from the bright-area polarization curves.

\subsubsection{Compositional similarity of bright and dark areas}

We now attempt to demonstrate polarimetrically that the dark areas have compositions similar to those of the bright areas, and differ chiefly in having a larger particle size; our method is to predict the polarization curve

$d_{1}^{a}$ from the curve $b^{\beta}$ from laboratory measurements on samples differing only in particle size. We define three empirical parameters, $L, M$, and $N$, which measure the variation of $\Phi_{1}, \Phi_{2}$, and $S \equiv \mathrm{dP} / \mathrm{d} \Phi\left(\Phi>\Phi_{2}\right)$ with a variation in $\mathrm{P}_{1}$ :

$$
\left.\begin{array}{rl}
L & \equiv \frac{\Delta \Phi_{1} /\left\langle\Phi_{1}\right\rangle}{\Delta P_{1} /\left\langle P_{1}\right\rangle} \\
M & \equiv \frac{\Delta \Phi_{2} /\left\langle\Phi_{2}\right\rangle}{\Delta P_{1} /\left\langle P_{1}\right\rangle} \\
N & \equiv \frac{\Delta S /\langle S\rangle}{\Delta \mathrm{P}_{1} /\left\langle P_{1}\right\rangle}
\end{array}\right\}
$$

where $\Delta$ indicates the variation between two polarization curves, and the angular brackets, \langle\rangle , the mean value. $L, M$, and $N$ were determined from two samples of red sandstone (Dollfus, 1957a) and two samples of limonite (Dollfus and Focas, 1966, Figure 14; here, $P_{1}$ for the coarse-grained sample was determined from the data points, rather than from the fitted curve, which does not quite fit near $\Phi_{1}$ ). Both materials yield polarization curves similar to those obtained for Mars. The limonite curves provide a better fit; we also note that limonite is a significant impurity in red sandstone. In Table 5, the derived values of $L, M$, and $N$ are displayed. The numerical values are not easily extracted from the laboratory curves because of the small differences involved. The values for red sandstone are somewhat more 
accurate (about 15 to $20 \%$ in absolute value), because of the larger $\Delta P_{1}$. Within their probable errors, the derived values of $L, M$, and $N$ for red sandstone and limonite are identical; we adopt the values for the former mineral in the following discussion.

Table 5. Values of the scaling parameters, L, M, and $\mathrm{N}^{*}$

\begin{tabular}{|l|c|c|c|}
\hline Material & $\mathrm{L}$ & $\mathrm{M}$ & $\mathrm{N}$ \\
\hline Red Sandstone & 0.37 & 0.16 & 1.01 \\
Limonite & 0.31 & 0.24 & 0.79 \\
\hline
\end{tabular}

* Probable errors for red sandstone are 15 to $20 \%$; for limonite, larger.

Our procedure is now to invert equations (14) and, with Table 5, to derive values of $\Phi_{1}, \Phi_{2}$, and $S$ for various curves from the values of these parameters for curve $b^{\beta}$. For example, for some curve $x$,

$$
\Phi_{1}(x)=\Phi_{1}\left(b_{1}^{a}\right)+L\left(\Delta P_{1} /\left\langle P_{1}\right\rangle\right) \Phi_{1}\left(b_{1}^{a}\right)
$$

where $\Delta$ and \langle\rangle refer to differences and means between the two curves $b_{1}^{a}$ and $x$. Despite the errors in estimating $L$ and $M, \Phi_{1}(x)$ and $\Phi_{2}(x)$ can be derived to within an accuracy of $1^{\circ}$ or $2^{\bullet}$, because $\Delta \Phi_{1}$ and $\Delta \Phi_{2}$ are small in all cases we will consider. On the other hand, the determination of $\mathrm{S}$ may in some cases be good only to $30 \%$ or so, since (1) measurements of $S$ have a larger associated error, and (2) $\Delta S$ may be comparable to $S$. 
With the above framework, we now make complete calculations for curves $d_{1}^{a}$ and $b^{a}$ from $b^{\beta}$. These curves are among the best determined, and also span a large range in $P_{1}$. The results are displayed in Table 6 for the case of uncorrected atmospheric polarization. It is obvious from Table 4 that atmospheric correction will result in no significant changes in Table 6. The good agreement between the derived and observed values for $b^{a}$ - which represents a bright area as $b^{\beta}$ does - provides a check on our method. To within the probable error discussed above, the derived and observed values for $d_{1}^{a}$ also agree. These agreements are essentially due to the small values of $\mathrm{L}, \mathrm{M}$, and $\mathrm{N}$; they indicate that both bright and dark areas have a similar chemical composition, and differ chiefly in particle size. The differences in polarization curve for dark areas at various times through the seasonal darkening are, as already mentioned, in just the same sense as the differences between bright and dark areas. Seasonal darkening in the dark areas, this discussion suggests, results from an increase in mean particle size without a change in chemical composition.

Table 6. Comparison of derived and observed polarization parameters

\begin{tabular}{|c|c|c|c|c|c|c|}
\hline & \multicolumn{2}{|c|}{$\Phi_{1}$} & \multicolumn{2}{c|}{$\Phi_{2}$} & \multicolumn{2}{c|}{ S } \\
\hline Curve & Derived & Observed & Derived & Observed & Derived & Observed \\
$\mathrm{b}^{\mathrm{a}}$ & $13^{\circ}$ & $12^{\circ}$ & $27^{\circ}$ & $27^{\circ}$ & 1.5 & 1.5 \\
$\mathrm{~d}_{1}^{\mathrm{a}}$ & 14 & 13 & 29 & 29 & 2.9 & 2.0 \\
\hline
\end{tabular}




\section{4. 5 Particle sizes}

We now attempt to estimate the mean radius of a dark-area particle. We assume that the chief mineral of importance for the polarization is goethite. The observed $P_{1}\left(b^{\beta}\right)=-9.5 \%$. The fine-grained limonite sample used above for calibration showed $P_{1}=-8 \%$ and consists of grains with $a<20 \mu$ (Dollfus and Focas, 1966); the coarse-grained sample showed $\mathrm{P}_{1}=-11 \%$, and $50 \mu \leq \mathrm{a} \leq 100 \mu$, approximately. However, the coarsegrained sample also showed some contamination by smaller grains $(a<50 \mu)$, which adhered to the larger grains and so were not separated out during sifting (Dollfus, private communication, 1966). From the comparison of the polarization curve for area $b^{\beta}$ with the limonite samples, we roughly estimate $\bar{a}\left(b^{\beta}\right) \simeq 25$ to $50 \mu$. (Note that since the limonite particles are not pure goethite they should yield somewhat larger particle sizes than deduced from the photometric data for goethite.) From the preceding discussion we see that a $40 \%$ increase in $\left|\mathrm{P}_{1}\right|$ corresponds roughly to an increase in $\bar{a}$ by a factor of 3 to 5 . The mean particle radius in the dark areas during the seasonal darkening would then be $125 \mu \leq \overline{\mathrm{a}}\left(\mathrm{d}^{\mathrm{a}}\right) \leq 375 \mu$, in good agreement with the value $\bar{a} \simeq 187 \mu$ found from photometry. Values of $\bar{a}\left(d^{\beta}\right)$, for the dark areas away from the maximum darkening, are intermediate between bright-area and maximum-darkening dark-area values, again in agreement with our previous conclusions. The agreement between the (largely independent) photometric and polarimetric arguments tends to strengthen each. 


\section{ON THE NATURE OF THE "BLUE HAZE"}

\subsection{An Alternative Hypothesis}

In previous discussion, we have alluded to the progressive loss of surface contrast observed toward shorter wavelengths on Mars. If interpreted as an atmospheric blue haze, these observations imply a haze layer with optical depth exceeding unity at $4500 \AA$, and probably increasing toward the ultraviolet. In its extreme form this hypothesis suggests that Mars is red only because of its blue haze, and that, throughout the visible, attempts at photometry of the Martian surface will be seriously confused by the influence of the atmosphere (cf. Kozyrev, 1955; Öpik, 1960).

We now present evidence that the loss of surface contrast in the blue is largely intrinsic to the surface and is not chiefly an atmospheric effect. Suppose the contrary is true, and initially assume that the atmospheric blue haze is a pure absorber, so that the light we see is ultimately derived from reflection off the surface. Then if $T$ is the optical depth of the haze layer, the observed contrast, $\Gamma$, between a bright and a dark area will be given by

$$
\Gamma=\frac{B_{b} e^{-\tau_{b}\left[\left(1 / \mu_{i}\right)+\left(1 / \mu_{r}\right)\right]}-B_{d} e^{-\tau_{d}\left[\left(1 / \mu_{i}^{\prime}\right)+\left(1 / \mu_{r}^{\prime}\right)\right]}}{B_{b} e^{-2 \tau_{b}\left[\left(1 / \mu_{i}\right)+\left(1 / \mu_{r}\right)\right]}},
$$

where $B_{b}$ and $B_{d}$ are the surface brightness of the bright and dark areas, respectively, in the absence of the haze layer, $\mu_{i}$ and $\mu_{r}$ denote the cosines of the angles of incidence and reflection for the bright areas, and $\mu_{i}^{\prime}$ and $\mu_{r}^{\prime}$ are corresponding quantities for the dark areas. By assumption, $B_{b}$ and $B_{d}$ differ significantly from one another. Only by very special and unlikely arrangements of $\tau_{b}$ and $\tau_{d}$ can we make $\Gamma$ approach 0 for all wavelengths 
below $4500 \AA$ and for all sets of angles of incidence and reflection. Such a blue haze would also imply very pronounced limb darkening at short wavelengths, contrary to observation, as Sha ronov (1957) has emphasized. Accordingly, we reject a pure-absorption blue haze.

A second blue haze model has the haze both scattering and absorbing sunlight so that the reflected radiation we observe below $4500 \AA$ originates chiefly from the haze itself. Significant contributions from the blue haze would still be expected at longer wavelengths, but with light reflected from the surface becoming increasingly more important. In this case, we would expect major differences in the polarization curves obtained at a variety of wavelengths. However, the negative branches observed at 5000, 5300,6000, $6300,8300,9500$, and 10,500 $\AA$ (Dollfus and Focas, 1966, Figures 4 and 5) are remarkably similar and are just what one would expect from a surface layer alone. Moreover, the positive branches differ only in amounts expected for a surface layer and a Rayleigh scattering layer with a brightness of some $7 \%$ at $4700 \AA$. Accordingly, we reject a (conservatively or nonconservatively) scattering model of the blue haze.

We have previously found that those goethite samples that match the spectrophotometry of Martian bright and dark areas at longer wavelengths lead to an intrinsic loss of surface contrast toward shorter wavelengths, with $\Gamma \sim 10 \%$ or less at $\lambda=4500 \AA$. We found the reason for this behavior to be the transition from multiple to single scattering for both particle-size distributions, as the domain of strong electronic absorption was approached. This hypothesis - that the loss of surface contrast on Mars toward the blue is largely an intrinsic property of the surface, and not an atmospheric effect - has been suggested much earlier (Menzel, 1926). In addition to arising naturally from an interpretation of the photometric data in terms of goethite, and avoiding the difficulties discussed in the preceding paragraph, it also helps to explain a number of otherwise very puzzling properties of the blue haze. 
At progressively shorter wavelengths, from red to ultraviolet, the contrast of the Martian polar cap relative to the rest of the disk steadily increases (see, e.g., Slipher, 1962). But the blue haze is supposed to markedly decrease such contrast toward shorter wavelengths. Must we imagine the blue haze as avoiding the polar regions? Alternatively, a uniform polar cloud, closely conforming to the outline of the polar cap, has been invoked. This cloud is entirely ad hoc, no other evidence having been offered in support of it. There is, for example, no polarimetric support (Dollfus, 1957a) for such a cloud. If there is no blue haze, the wavelength dependence of the polar cap contrast is then much easier to understand as an intrinsic surface effect; the reflectivity of ice is essentially constant in the visible while the reflectivity of bright and dark areas declines markedly to shorter wavelengths.

In the middle ultraviolet, the putative blue haze is expected to be very opaque. Yet rocket ultraviolet photometry of Mars reveals a wavelength dependence of scattering that obeys the Rayleigh law, and yields a surface pressure in good agreement with infrared-spectrometric and Mariner 4 occultation values (Evans, 1965). On these grounds, Evans (1965) has also argued that there is no blue haze.

\subsection{The "Blue Clearings"}

However, there is one observation that seems clearly to imply the existence of an atmospheric blue haze. There are instances of "blue clearing, "the occasional appearance of the usual surface features - although with reduced contrast - in photographs taken in the blue. Above we have seen that typical dark areas should have a contrast of only several percent in the blue and so will be marginally detectable. Under these circumstances a number of factors - quite apart from a hypothetical atmospheric layer - will influence the detectability of surface features on Mars and lead to a time variability in the detectability of features in the blue. Dark areas will most easily be discovered in the blue when (1) the seeing is good, (2) the dark areas 
have a large angular extent in two dimensions, (3) the contrast is high at longer wavelengths, and (4) there are few or no Martian clouds. When the seeing is poor, our ability to discern low-contrast features declines markedly. Below we show that each of the foregoing factors is important and that blue clearings are correlated with at least some of them. If both the blue "haze" and the blue "clearings" can be generated by these mechanisms, an atmospheric haze layer is not their only explanation. In view of the discussion in the preceding subsection, we consider it unlikely that an atmospheric haze layer is playing an important role.

Observers who have attempted to see or photograph the low-contrast features of Mercury are seriously affected by seeing, and generally succeed in obtaining the sought-for features only at the moments of very best seeing. Thus for Mercury there are phenomena that might be described as hazes and clearings related to seeing; such phenoma can also be expected for Mars. In general, those surface features which have large dimensions in both latitude and longitude can be expected to be the least affected by seeing smearing.

The larger the contrast of a feature in the yellow, the larger will be its contrast in the blue. Accordingly, we expect more blue clearings during the seasonal darkening. Focas (1966) has found such a correlation. This illustrates the importance of factor (3).

We now present several pieces of evidence illustrating the importance of factors (2) and (3). We predict that blue clearings would be most frequent when dark areas with large dimensions and high contrast, such as Syrtis Major, are near the center of the disk.

In Figure 21 (Slipher, 1962) is plotted the fraction of photographs showing significant blue clearing as a function of the longitude of the central meridian. A striking peak in the histogram for the longitudes of Syrtis Major can be seen. It is instructive to compare the longitude interval 240 to $320^{\circ}$ 

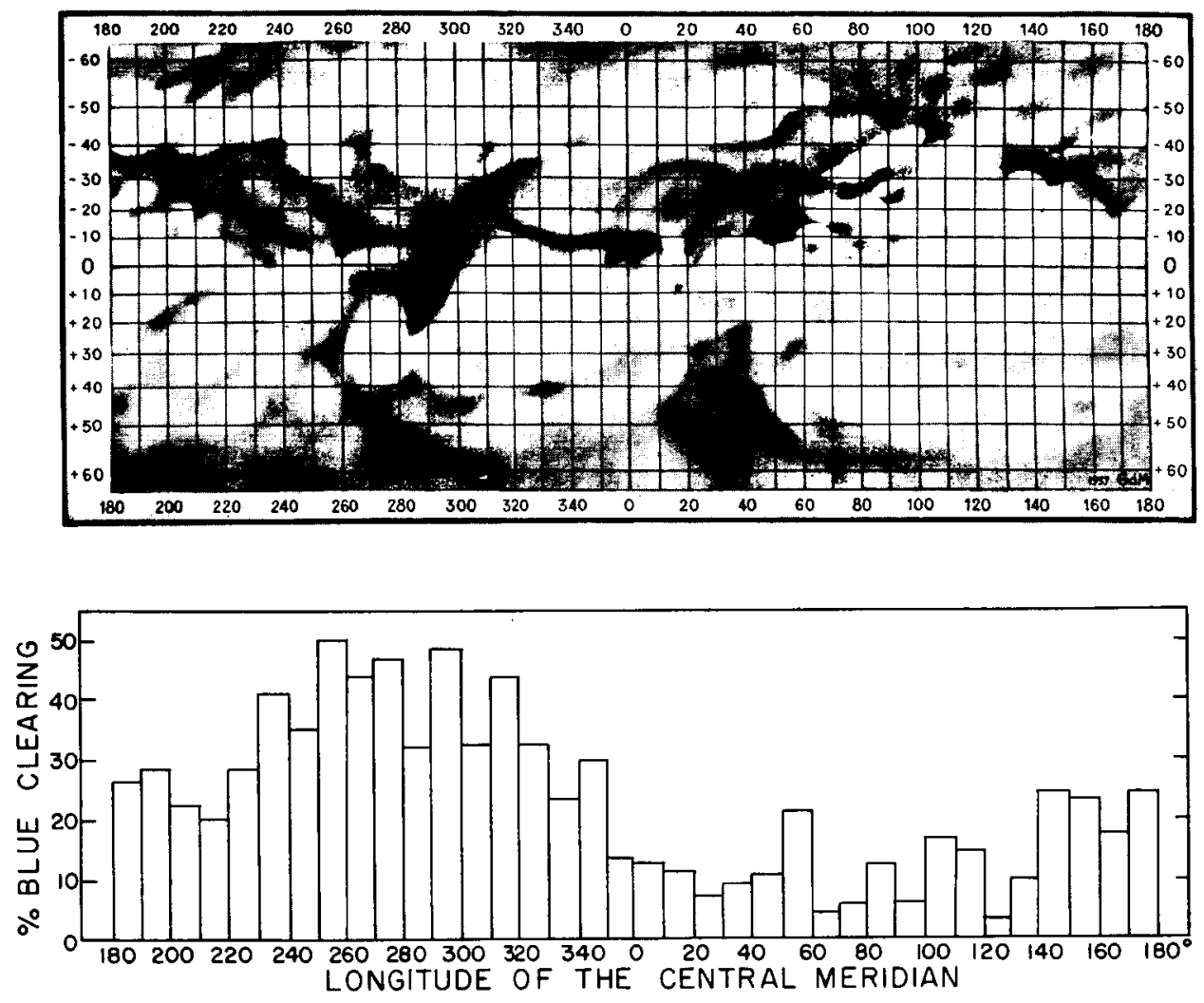

Figure 21. Correlation of frequency of blue clearing with longitude of the central meridian. The histogram should be compared with the map of Mars above, which is to the same longitude scale. (After Slipher, 1962.) 
with the interval 320 to $20^{\circ}$. While the principal dark areas between 320 and $20^{\circ}$, Sabaeus Sinus and Meridiani Sinus, are quite dark, they are also long and narrow, and lack any great extent in latitude. We expect, therefore, fewer blue clearings for 320 to $20^{\circ}$ than for 240 to $320^{\circ}$, as is observed. Comparing the histogram of Figure 21 with the map of Mars (remembering that Mercator projection overemphasizes the areas of polar regions, relative to what is actually seen in observing the planetary disk), we find that other regions follow this pattern.

Slipher (1962, p. 40) notes the remarkable fact that the degree of blue clearing differs significantly as observed by different observatories (Lowell, Lamont-Hussey, and Mt. Stromlo) on the same night. The Lowell and Lamont-Hussey photographs were each obtained with a blue-corrected refractor, on the same type of photographic emulsion, and processed in a similar manner. But we can also understand this circumstance. Not only were the seeing conditions different at the different observatories, but also the Martian central meridians observed differed by some $120^{\circ}$, and regions of very different albedo and extent were being viewed.

There is the possibility that clouds in the Martian atmosphere can sometimes play a role in the degree of blue clearing. The Martian clouds generally have a low reflectivity, so it is in the blue, where the surface also has a low albedo, that the clouds should be most evident and make their largest contribution to the observed radiation. On a very cloudy day on Mars, the clouds could effectively mask surface features or make it difficult to identify regions of obse rved contrast with such regions at longer wavelengths. Most of the examples of blue clearings or lack of clearings we have been concerned with above refer to relatively cloud-free times. There are socalled blue clouds (cf. Kellogg and Sagan, 1961) on Mars.

At times when the Martian atmosphere has many blue clouds, spurious blue clearings may be generated; features of fairly high contrast may appear, but they will not necessarily be related closely to features in the yellow or 
red. A similar explanation of blue clearings has been suggested by Dollfus (1964, private communication).

Future work - e.g., in seeking high-contrast blue clearings, or in correlating blue contrasts with seeing - are clearly needed to test the hypotheses presented in this section. 


\section{DISCUSSION OF RESULTS}

\subsection{Summary of Conclusions}

We first summarize our basic conclusions and the data on which they are based. The sharp rise in reflectivity between 4500 and $6500 \AA$ (Section 2.1.2), and the distinct negative branch at small phase angles in the polarization curve (3.4) imply independently that both bright and dark areas are composed of fine powders or of extremely porous rocks. This result is consistent with the low thermal inertia of these areas, and with the low radar reflectivity of the bright areas. The existence of Martian clouds that arise in the bright areas and have photometric properties similar to those of the bright areas suggests that the surface material is a powder rather than an extremely porous rock.

The spectra of the bright and dark areas a re controlled between $3000 \AA$ and $11,000 \AA$ mainly by their ferric oxide content. This is indicated by the low, nearly constant reflectivity between 3000 and $4500 \AA$, by the steep slope between 4500 and $7000 \AA$, and by the plateau between 7000 and $11,000 \AA$; all fit well by goethite once a particle size is chosen that is appropriate to the absolute value of the reflectivity at one wavelength (Sections 2.1.3, 2.1.4). There appears to be no other geochemically abundant material strongly enough absorbing in the blue to reproduce this spectrum. Ferric oxide polyhydrates also match the polarization curves of bright and dark areas extremely well (Sections 3. 1, 3.4.2). Because of calibration problems in normalizing Martian to solar spectra, and because the feature has a wideband low-strength character, present observations are not sensitive enough to test the presence of ferric oxides by searching for the limonite band near $9000 \AA$, which arises from absorption by $\mathrm{Fe}_{2} \mathrm{O}_{3}$ (Section 2.2.1). 
The large reflectivity decline between 2.4 and $3.1 \mu$, in both bright and dark areas, indicates the presence of significant quantities of water of hydration in both areas (Section 2.2.3). Neither Martian ice clouds nor surface carbonates seem capable of accounting for these observations. Com-

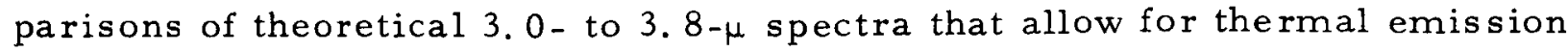
with the observed spectra imply at least one molecule of water of hydration per $\mathrm{Fe}_{2} \mathrm{O}_{3}$ moiety. A small quantity of additional water may be present, either bound or adsorbed to ferric oxides or to other constituents. The peaking of the reflectivity curve near $1.4 \mu$ also suggests the presence of water of hydration, since the water opacity begins to become appreciable at slightly longer wavelengths (Section 2.2.3). The polarimetry yields a low value of the real part of the refractive index, again implying the presence of at least one molecule of water of hydration per $\mathrm{Fe}_{2} \mathrm{O}_{3}$ (Section 3.4.2).

That ferric oxides are a major constituent of the bright areas, and not a thin patina of desert varnish, follows from the approximate agreement between the particle sizes implied by comparison of photometry of Mars and of pure goethite samples with the particle sizes found from the Stokes-Cunningham fallout times of observed yellow clouds, and from the bright-area thermal inertias (Section 2.1.5). The same conclusion follows from a comparison of the refractive index required to account for the polarimetry with that of a composite silicate with a goethite patina (Section 3.4.2).

That ferric oxides are also a major constituent of the dark areas follows from the near identity of the refractive indices of bright and dark areas; this agreement derives from the very low contrast in the blue, violet, and ultraviolet (Sections 2.1.3, 2. 1.4), and from the fact that the polarization curve for the dark areas can be derived from that for the bright areas merely by increasing the mean particle size (Section 3.4.4). Even during the seasonal darkening of the dark areas, their index of refraction remains almost the same as for the bright areas. 
Since these considerations indicate that goethite is a principal constituent of the surface, a first approximation to mean particle radii, $\bar{a}$, on Mars can be obtained by matching laboratory and Martian reflectivities (Section 2. 1. 5). The bright areas are characterized by $\bar{a} \simeq 25 \mu$; the dark areas outside the seasonal darkening, by $\bar{a} \simeq 100 \mu$; and the dark areas during the seasonal darkening, by $\bar{a} \simeq 200 \mu$. Yellow-cloud fallout times (Section 2.1.5), surface thermal inertias (Section 2.1.5), and polarimetry (Section 3.4.5) give similar results. Both the photometry and, particularly, the polarimetry, indicate that the principal event of the wave of darkening is a change in mean particle size, by a factor $\sim 2$, with no substantial change in composition.

Samples of pure goethite that match the observations at other wavelengths give too high a reflectivity in the region just beyond $1.1 \mu$. Without changing any of the other results, agreement with observation can be secured by the addition of small quantities of $\mathrm{Fe}_{3} \mathrm{O}_{4}$ or $\mathrm{MgO}$, both reasonable materials by terrestrial or meteoritic analogy (Section 2.2.2). Somewhat larger amounts of these materials are required for the dark areas than for the bright areas. The presence of these materials will also decrease the detectability of the limonite band.

This model also accounts for the los of surface contrast toward the blue in a very natural way, without any necessity for invoking a blue haze (Section 4. 1). The similarity of the negative branches of the polarization curves at various wavelengths, the enhanced short-wavelength visibility of the polar caps, the ultraviolet spectra, and other evidence all imply that indeed the re is no blue haze (4.1). It is suggested that seeing p'ays an important role in the occurrence of blue clearings, with good blue seeing, no Martian clouds, and large and contrasting dark areas enhancing the prospects for detectable blue clearings (Section 4.2).

Thus, the bright and dark areas of Mars are composed in significant part of limonite; i. e., goethite $\left(\mathrm{Fe}_{2} \mathrm{O}_{3} \cdot \mathrm{H}_{2} \mathrm{O}\right)$ is a major constituent, with variable amounts of bound and adsorbed water, as well as other minerals, 
probably present. The dark-area particles are generally larger than the bright-area particles, and the dark-area particle sizes increase during the seasonal darkening.

\subsection{Objections to the Model}

We next discuss various a rguments that have been advanced against goethite being a principal constituent of the bright areas. We have already seen that the absence of a well-defined limonite band in Martian spectra presents no basic contradiction with the conclusions of the present paper. Coulson, Bouricius, and Gray (1965) have examined the angular scattering properties of samples of pulverized goethite, and have found a strong backward lobe in the scattering diagram that is not found for Mars. However, this comparison is invalid. The laboratory measurements refer to a fixed angle of incidence and varying angles of reflection, while the astronomical measurements refer to varying angles of both incidence and reflection as a function of position on the Martian disk. For Mars, the difference between angles of incidence and reflection is the same, at a given phase angle, for all points on the disk. It is only at $\Phi=0^{\circ}$ that the effect should be prominent. However, it is not a unique indicator of limonite; many materials show similar backscattering. It is significant that Coulson et al. find a strong backscattering lobe for all angles of incidence with little variation in reflectivity. We also note that the laboratory measurements of Coulson et al. are probably dependent on the geometrical properties of the sample. They smooth down the sample and, in so doing, tend to orient the normals to the grain surfaces parallel to the local surface normal. On Mars a more random distribution of orientation is expected.

An important theoretical question concerns the thermodynamic stability of goethite under Martian conditions of temperature and humidity. Adamcik (1963), and Schmalz (1959) have come to opposite conclusions on this question, chiefly because of differences in their choices of thermodynamic variables. In a more recent and careful analysis, Fish (1966) finds a substantial uncertainty as to the correct values of the thermodynamic 
variables, and attempts to bracket the true values. He finds that for mean diurnal surface temperatures, $\overline{\mathrm{T}} \geq 250^{\circ} \mathrm{K}$, goethite will be unstable under the low water-vapor abundances of the Martian atmosphere. Fish concludes that goethite on Mars should be converted almost entirely to hematite. But so strong a conclusion cannot be drawn from these arguments. There are three principal problem areas: the choice of an appropriate mean temperature for the goethite; the question of humidities in the immediate subsurface environment; and the distinction between conclusions drawn from the rmodynamic equilibrium and from absolute reaction-rate kinetics. Sinton and Strong (1960) find that typical noontime equatorial surface temperatures are around $300^{\circ} \mathrm{K}$, dropping by twilight to about $200^{\circ} \mathrm{K}$. The nighttime temperatures will be no more than some tens of degrees lower, depending on the thermal inertias. Both yearly maximum and yearly mean temperatures will be less at other latitudes. Below a depth $\sim 1 \mathrm{~cm}$, the diurnal temperature variations are essentially completely damped; except at the very surface, the ground is at a constant temperature of about $220^{\circ} \mathrm{K}$ at the equator, and somewhat less at other latitudes. At $\overline{\mathrm{T}} \leq 200^{\circ} \mathrm{K}$ and with the spectroscopic water-vapor abundance, we are in the goethite stability field, according to the calculations of Fish. Thus, there appears to be a large reservoir of water, in the form of wate $r$ of hydration, at depths below $1 \mathrm{~cm}$; it is this water, rathe $r$ than the instantaneous atmospheric water-vapor content, that controls Martian humidity. However, it is the top centimeter that is viewed in the visible and infrared, and not the constant $220^{\circ} \mathrm{K}$ level. Since the equilibrium water-vapor pressure over a hydrated mineral increases exponentially with increasing temperature, we might first be tempted to choose $\overline{\mathrm{T}}$ nearer $300^{\circ} \mathrm{K}$ than $200^{\circ} \mathrm{K}$ as a topographic surface mean; this is, in fact, what $\mathrm{F}$ ish has done. However, a grain of goethite exposed to an environment in which the watervapor partial pressure is far below the equilibrium vapor pressure will dehydrate and outgas from the surface inward. It is not certain that dehydrated goethite is crystallographically necessarily hematite. Furthermore, dust grains are frequently moved around on Mars by the winds, so that a given grain initially in the top layer of the soil rathe $r$ quickly returns to a depth of $1 \mathrm{~cm}$ or more, where the conditions a re favorable for goethite stability. Under these circumstances, the time to reach equilibrium becomes a 
critical factor and kinetics may be on the side of the stability of goethite grains in the top layer: the time to reach equilibrium is probably quite long since diffusion of wate $r$ molecules from the interior of grains is involved in the dehydration process.

In addition, water may occasionally be available to provide favorable stability conditions near the surface: the frost point is reached before sunrise on Mars and in fact dawn hazes are observed. Precipitated water may lie on the Martian surface each day; water trapped in the subsurface structure as the temperature rises will greatly increase the local subsurface humidities. In this context it is interesting to note that some terrestrial goethite deposits appear in places where thermodynamic equilibrium is apparently violated (R. Siever, private communication, 1967). Clearly, much work remains to be done on the question of goethite stability.

Finally, van Tassel and Salisbury (1964) have objected to large quantities of iron oxides on Mars essentially on the grounds that (by terrestrial analogy and from cosmic abundances) large quantities of silicates should be present. They claim that the mean density of Mars is below what one would expect with $(\mathrm{Fe} / \mathrm{Si}) \geq 1$. This is true if the iron is present as the metal, as in a planetary core. But the moment of inertia of the planet and the negative results on trapped particles and magnetic fields obtained by Mariner 4 counterindicate an appreciable iron core. Iron combined with oxygen, as in limonite, has a density close to the uncompressed density of Mars. The results of the present paper refer, however, only to the surface layers of Mars; they are certainly compatible with the presence of silicates, only provided that goethite is a major constituent. However, more surface iron does seem to be present on Mars than on Earth, a conclusion discussed below. 


\subsection{Some Implications}

We now briefly explore some further implications of our results. We have found sizable quantities of bound or adsorbed water in the Martian surface layers. Strictly speaking, the photometry, polarimetry, and spectroscopy pertain only to the top millimeter or so; but when account is taken of the stirring of the dust by winds, we find that our results must apply to some greater depth. Furthermore, radar observations indicate that the bright areas are pulverized to a depth of at least $1 \mathrm{~m}$ (Sagan and Pollack, 1967c); presumably, the composition does not vary over this depth. The mean atmospheric water-vapor content is $\sim 10^{-3} \mathrm{gm} \mathrm{cm}^{-2}$ (Schorn, Spinrad, Moore, Smith and Giver, 1967), comparable to that in a goethite specimen $10 \mu$ thick. Thus, practically all the water on Mars is present subsurface; the amount is at least $10^{2} \mathrm{gm} \mathrm{cm}^{-2}$, and may be much larger. The water present on the surface of the Earth is believed to have been altogether outgassed from the interior by volcanism and other plutonic activity (see, e.g., Cameron and Brancazio, 1964). While the re may be some contribution from the infall of cometary debris on Mars, the bulk of the water there should also be endogenous. Thus, some appreciable outgassing of Mars appears to have occurred. Compared to the several-km-thick equivalent layer of water on Earth, the minimum Martian equivalent layer of meters that we have deduced implies an outgassing rate at least $0.1 \%$ that of Earth and possibly much larger. If the bulk of the $\mathrm{N}_{2}$ outgassed in geological time is present in the contemporary terrestrial atmosphere, assuming initial conditions on the two planets to have been similar, this implies a Martian outgassing rate of $>1 \mathrm{mb}$ of $\mathrm{N}_{2}$ in geological time. Even if $\mathrm{N}_{2}$ has never escaped from Mars, this implies no contradiction with the present atmospheric composition (Sagan and Pollack, 1967b). With $\sim 10$ atm $\mathrm{CO}_{2}$ equivalent in the terrestrial sedimentary column (Hutchinson, 1954) and the same assumptions, a Martian outgassing rate of $>100-\mathrm{mb} \mathrm{CO}_{2}$ over geological time is implied. The contemporary atmosphere is unlikely to have more than $\sim 10-\mathrm{mb} \mathrm{CO}_{2}$, but surface carbonate deposits can be expected on Mars as on the Earth. We also note that during the past $4.5 \times 10^{9}$ years, appreciable quantities of water may have been lost from Mars (depending on the history of the atmospheric structure and exospheric temperature), but 
not from Earth. Because of surface reaction and escape, no contradiction with the present Martian atmosphere is the reby generated, but a greater outgassing rate would be implied.

It is interesting that the iron on the Martian surface is almost entirely in the oxidized form $\mathrm{Fe}_{2} \mathrm{O}_{3}$, rather than such more reduced forms as $\mathrm{Fe}_{3} \mathrm{O}_{4}$ or FeO. By comparison, the oxidation state of terrestrial iron of the crust is close to that of magnetite, while stony meteorites generally lie between $\mathrm{FeO}$ and Fe (see, e.g., Birch, Schairer and Spicer, 1942). One possible explanation for the high degree of oxidation on Mars is the photodissociation of water vapor, the escape of hydrogen to space, and the oxidation of the Martian surface by the oxygen left behind (Wildt, 1934; Sagan, 1966). The additional outgassed water required is some three times that discussed in the previous paragraph.

The silicon content of the Martian surface material is at most comparable to that of iron. The photometric and polarimetric evidence excludes any great preponderance of silicon compounds. By contrast, the crust of the Earth is composed principally of silicon and oxygen, with some aluminum and relatively little iron. The mantle seems to contain iron and silicon in approximately equal amounts; a similar statement holds for stony meteorites, while $(\mathrm{Fe} / \mathrm{Si}$ ) is increased in iron meteorites (Birch et al, 1942). Thus the iron-tosilicon ratio on the Martian surface is comparable to the undifferentiated cosmic abundances.

Two possiblities have been suggested (Sagan, 1966) to explain the higher surface (Fe/Si) on Mars than on Earth. During differentiation of the Earth, a major fraction of the iron has migrated toward the core. Mars, on the other hand, has probably experienced less differentiation and has, at best, a very small core. In this case, the formation of a crust may have been inhibited and the surface composition of Mars may be then roughly characteristic of the gross composition of the planet. Alternatively, the iron content of the Martian surface is larger than that of the immediately underlying layers, permitting some substantial planetary differentiation. The iron-rich 
surface layers can then be attributed to meteoritic infall, Mars being much closer to the asteroid belt than is the Earth. Scaling the terrestrial micrometeorite-infall rate (Parkin and Tilles, 1967) by a factor of 10 , we find an accumulation over $4.5 \times 10^{9}$ years of between 2 and $200 \mathrm{~m}$. Because of atmospheric drag, these particles suffer negligible ablation in entering the Martian atmosphere, fall gently to the surface (cf. Öpik, 1958), and produce no craters. The iron-to-silicon ratio of meteorites is, as mentioned above, within the range deduced for Mars.

The Mariner 4 photographs imply that about $15 \%$ of Mars is covered by craters with diameters in excess of $9 \mathrm{~km}$. Typical depth-to-diameter ratios for newly formed craters are $\sim 0.1$. From this source alone, then, an average depth of some $100 \mathrm{~m}$ has been pulverized. (This may be in agreement with the mean depth of cover from micrometeorites, and suggests that some substantial filling of Martian craters by micrometeorites has occurred. The craters do have a pronounced flat-bottomed filled-in appearance.) Because limonite is more friable than iron-poor silicates, it will constitute a larger fraction of the rock flour created by meteorite impact. In turn this rock flour is lifted by the winds, and will preferentially cover the surface (Rea, $1965)$.

The compositional similarity between bright and dark areas can be caused by the smaller planetary differentiation or by the larger micrometeoritic infall. Winds will certainly tend to homogenize the surface layers of the planet. The particle sizes of the bright-area material are such that the expected Martian winds will lift these particles and carry them fair distances. The larger darkarea particles will only saltate or bounce along the surface. Those particles that can be carried by the wind will constitute the surface layers; this provides a causal relation to explain the particle sizes deduced. The segregation of particle sizes between bright and dark areas can be understood quantitatively in terms of elevation differences. Seasonal exchange of material by winds between the bright and dark areas offers a natural explanation of the seasonal photometric and polarimetric changes, which we have seen to be due to variations in particle sizes rather than composition. These matters are the subject of a paper to be published elsewhere. 


\subsection{Recommendations for Future Work}

Clearly much needs to be done both observationally and in the laboratory. Studies of the influence of impurities, $\tilde{\omega}_{0}$, etc., on the "limonite" band are needed. It would be worthwhile to see what parameters surface limb darkening depends upon - e.g., flat or open structure, $\widetilde{\omega}_{0}$, etc. In this sense, total reflectivity measurements may perhaps be preferred over direct measurements of $K$ until we know how $K$ depends on $y$, the openness of structure of the sample. Total reflectivity measurements are more likely to be independent of $y$. If possible, total reflectivity measurements of a sample on a sphere would be preferred, so as to permit a direct comparison of the laboratory measurements with astronomical values of the geometric albedo. More studies of the reflectivity of goethite, hematite, magnetite, $\mathrm{MgO}$, and water of hydration, both alone and in various combinations are needed from $0.3 \mu$ to $4 \mu$. When measurements are performed in a wavelength region where the reflectivity is high, care must be taken to ensure sufficient sample thickness - scattering for $\tilde{\omega}_{0}$ close to 1 reguires quite high optical thicknesses; the $T=\infty$ case must be approached. Sensors at the bottom of the sample may be useful in this regard. For measurements in wavelength regions of low reflectivity, great care must be paid to scattered light in the instrument, which can cause anomalously high reflectivities.

In laboratory polarization work it would b useful to fix the value of the reflectivity to agree with that of the bright areas and then to vary the index of refraction, keeping the negative contribution the same; in this way it would be possible to determine more exactly the index of refraction of the bright areas of Mars. It would also be useful to perform experiments with a constant thickness of goethite over a variable sized core of quartz to find the dependence of the negative polarization contribution on particle size. This result should be obtained for various indices of refraction, reflectivities, etc. In so doing we may see if the depth of the negative branch of the polarization curve gives us information on particle size or at least bounds on it, and the extent to which the degree of openness also influences the negative branch. 
On the observational side, more work is needed on values of $K$ and on the geometric albedos, particularly in the region from $7000 \AA$ to $4 \mu$. In the $7000-\AA$ to $11,000-\AA$ range, great care must be paid to normalizing correctly the Martian spectra relative to the Sun, perhaps by use of a G2V star. It is also very important that simultaneous observations of extinction, if possible with the aid of stars close to Mars, be made because of the time variability and spatial inhomogeneity of the water-vapor content. Surface limbdarkening measurements to find values of $a$ and $\gamma$ at various wavelengths would also be quite valuable. Finally, searches for a connection between "blue clearings" and improvements in seeing would be of interest. 


\section{ACKNOWLEDGMENTS}

We are very grateful to Dr. R. Siever for informative discussions on the crystallography of goethite and its terrestrial environments; to Drs. A. Dollfus and J. Focas for extended discussions concerning their research; to Drs. W. Hovis, W. Sinton, W. Tull, and R. Younkin for advance copies of their papers; to Drs. G. de Vaucouleurs, D. Rea, and A. T. Young for helpful conversations and to Mrs. Elinore Green and Miss Leona Dryden for assistance with calculations and the construction of the graphs. 


\section{REFERENCES}

AD AMCIK, J. A.

1963. The water vapor content of the Martian atmosphere as a problem of chemical equilibrium. Planet. Space Sci., vol. 11, pp. $355-359$.

BIRCH, F., SCHAIRER, J. F. and SPICER, H. C., eds.

1942. Handbook of Physical Constants. Waverly Press, Baltimore, 325 pp.

CAMERON, A. G. W. and BRANCAZIO, P. J.

1964. The Origin and Evolution of Atmospheres and Oceans. John Wiley \& Sons, New York, $314 \mathrm{pp}$.

COULSON, K. L., BOURICIUS, G. M. B. and GRAY, E. L.

1965. Effect of surface reflection on radiation emerging from the top of a planetary atmosphere. General Electric Space Sciences Laboratory Rept. R65SD64.

DANIELSON, R. E., GAUSTAD, J. E., SCHWARZSCHILD, M. , WEAVER, H. F. and WOOLF, N. J.

1964. Mars observations from Stratoscope II. Astron. Journ., vol. 69, pp. 344-352.

DOLLFUS, A.

1956. Polarisation de la lumière renvoyée par les corps solides et les nuages naturels. Ann. d'Astrophys., vol. 19, pp. 83-113.

1957a. Étude des planètes par la polarisation de leur lumière. Ann. d'Ap. Súppl. No. 4, 114 pp; also available as NASA Technical Translation NASA - T T - F -188 .

1957b. Étude photométrique des contrées sombres sur la planète Mars. Comptes Rendus, vol. 244, pp. 1458-1460.

1957c. Propriétés photométrique des contrées désertique sur la planète Mars. Comptes Rendus, vol. 244, pp. 162-164.

DOLLFUS, A. and FOCAS, J. H.

1966. Polarimetric study of the planet Mars. Air Force Cambridge Laboratories Final Rept. on Contract AF-61(052)-508, 28 pp. 
EVANS, D. C.

1965. Ultraviolet reflectivity of Mars. Science, vol. 149, pp. 969-972. FISH, F. F., Jr.

1966. The stability of goethite on Mars. Journ. Geophys. Res., vol. 71 , pp. $3063-3068$.

FOCAS, J. H.

1961. Étude photométrique et polarimétrique des phénomenenes saisonniers de la planète Mars. Ann. d'Astrophys., vol. 24, pp. 309-325.

1966. Transparence de l'atmosphere martienne et visibilit'e des details de la surface dans le bleu et ultraviolet. Presented at the Cospar meeting in Vienna, May.

FORSYTHE, W. E.

1954. Smithsonian Physical Tables, 9th rev. ed. Smithsonian Institution Press, Washington, D. C., 827 pp.

GOLDSTEIN, S., ed.

1938. Modern Developments in Fluid Dynamics, vols. 1 and 2.

Clarendon Press, Oxford, England.

GRAY, D. E., ed.

1963. American Institute of Physics Handbook, 2nd ed. McGraw-Hill Book Co. Inc., New York.

GREEN, H. L. and LANE, W. R.

1957. Particulate Clouds: Dusts, Smokes and Mists. D. Van Nostrand, New York, p. 54.

HOVIS, W. A., Jr.

1965. Inf rared reflectivity of iron oxide minerals. Icarus, vol. 4, pp. $425-430$.

HOVIS, W. A. Jr. and CALLAHAN, W. R.

1966. Infrared reflectance spectra of igneous rocks, tuffs, and red sandstones from 0.5 to $22 \mu$. Journ. Opt. Soc. Amer., vol. 56, pp. 639-643.

HOPFIELD, J. J.

1966. Mechanism of lunar polarization. Science, vol. 151, pp. 13801381. 
VAN DE HULST, H. C.

1957. Light Scattering by Small Particles. John Wiley \& Sons, New York, 470 pp.

HURWICH, H. H. and JAMIESON, D.

1965. Remarks made at the Conference on Remote Investigation of

Martian Biology, Cambridge, Mass., January.

HUTCHINSON, G. E.

1954. The biochemistry of the terrestrial atmosphere. In The Earth as a Planet, vol. 2 of The Solar System, ed. by G. P. Kuiper, Univ. of Chicago Press, Chicago, pp. 371-433.

IRVINE, W. M. and POLLACK, J. B.

1967. Infrared optical properties of water and ice spheres. Icarus, in press.

KELLOGG, W. W. and SAGAN, C., eds.

1961. The Atmospheres of Mars and Venus. Nat. Acad. Sci. - Nat. Res. Council Publ. 944, 151 pp., Washington, D. C.

KOZYREV, N. A.

1955. Publ. Crimean Astrophys. Obs., vol. 15, pp. 147-152. KUIPER, G. P.

1952. Atmospheres of The Earth and Planets, 2nd ed. Univ. of Chicago Press, Chicago, Chap. 12, pp. 306-405.

1957. Visual observations of Mars. Astrophys. Journ., vol. 125, pp. $307-317$.

1964. Infrared spectra of stars and planets. IV. The spectrum of Mars, $1-2.5$ microns, and the structure of its atmosphere. Communications of the Lunar and Planetary Laboratory, Univ. of Arizona, vol. 2, No. 31, pp. 79-112.

LEOVY, C.

1966. Note on the rmal properties on Mars. Icarus, vol. 5, pp. 1-6. ÖHMAN, Y.

1955. A tentative explanation of the negative polarization in diffuse reflection. Stockholma Observatoriums Annales, vol. 18, No. 8. 
ÖPIK, E. J.

1958. Physics of Meteor Flight in the Atmosphere. Interscience

Publishers Inc., New York, 174 pp.

1960. The atmosphere and haze of Mars. Journ. Geophys. Res., vol.

65, pp. 3057-3063.

PARKIN, D. W. and TILLES, D.

1967. Influx measurements of extrater restrial material. Smithsonian Astrophys. Obs. preprint, $40 \mathrm{pp}$.

POLLACK, J. B.

1967. Rayleigh scattering in an optically thin atmosphere and its application to Martian topog raphy. Icarus, vol. 7, pp. 42-46.

POLLACK, J. B. and SAGAN, C.

1965a. The microwave phase effect of Venus. Icarus, vol. 4, pp. $62-103$.

1965b. The infrared limb darkening of Venus. Journ. Geophys. Res., vol. 70, pp. $4403-4426$.

REA, D. G.

1965. Some comments on 'The composition of the Martian surface. Icarus, vol. 4, pp. 108-109.

REA, D. G. and O'LEARY, B. T.

1965. Visible polarization data of Mars. Nature, vol. 206, pp. 1138 1140.

SAGAN, C.

1966. Mariner IV observations and the possibility of iron oxides on the Martian surface. Icarus, vol. 5, pp. 102-103.

SAGAN, C., and KELLOGG, W. W.

1963. The terrestrial planets. Ann. Rev. Astron. Astrophys., vol. 1, pp. 235-266.

SAGAN, C., PHANEUF, J. P. and IHNAT, M.

1965. Total reflection spectrophotometry and thermogravimetric analys is of simulated Martian surface materials. Icarus, vol. 4, pp. 43-61. 
SAGAN, C. and POLLACK, J. B.

1967a. Anisotropic nonconservative scattering and the clouds of Venus. Journ. Geophys. Res., vol. 72, pp. 469-477.

1967b. Elevation differences on Mars. Smithsonian Astrophys. Obs. Spec. Rep. No. 224, 45 pp.

1967c. Radio evidence on the structure and composition of the Martian surface. To be published; also in Journ. Res. Nat. Bur. Standards, vol. 69D, p. 629, 1965.

SAGAN, C., POLLACK, J. B. and GOLDSTEIN, R. M.

1967. Radar Doppler spectroscopy of Mars. I. Elevation differences between bright and dark areas. Astron. Journ., vol. 72, pp. $20-34$.

SCHMALZ, R. F.

1959. A note on the system $\mathrm{Fe}_{2} \mathrm{O}_{3}-\mathrm{H}_{2} \mathrm{O}$. Journ. Geophys. Res., vol. 64, pp. 575-579.

SCHMIDT, I.

1959. Visual problems in observing the planet Mars. Proc. of Lunar and Planetary Exploration Colloquium, vol. I, No. 6, pp. 19-22.

SCHORN, R. A. , SPINRAD, H., MOORE, R. C. , SMITH, H. J. and GIVER, L. P.

1967. High-dispersion spectroscopic observations of Mars. II. The water-vapor variations. Astrophys. Journ., vol. 147, pp. $743-752$.

SHARONOV, V. V.

1957. On the role of true absorption in the Martian atmosphere. A. J. USSR, vol. 34, p. 547.

1961. A lithological interpretation of the photometric and colorimetric studies of Mars. Soviet Astron. - A. J., vol. 5, pp. 199-202.

SINTON, W. M.

1967. On the composition of Martian surface materials. Icarus, vol. 6, pp. $222-228$. 
SINTON, W. M. and STRONG. J.

1960. Radiometric observations of Mars. Astrophys. Journ., vol. 131, pp. 459-469.

SLIPHER, E. C.

1962. The Photographic Story of Mars, ed. by J. S. Hall. Sky

Publ. Corp., Cambridge, Mass., and Northland Press, Flagstaff, Arizona, $168 \mathrm{pp}$.

VAN TASSEL, R. A. and SALISBURY, J. W.

1964. The composition of the Martian surface. Icarus, vol. 3, pp. 264269.

TULL, R. G.

1966. The reflectivity spectrum of Mars in the near-infrared. Icarus, vol. 5, pp. 505-514.

DE VAUCOULEURS, G.

1954. The Physics of the Planet Mars. Faber and Faber, Ltd., London, $365 \mathrm{pp}$.

1964. Geometric and photometric parameters of the terrestrial planets. Icarus, vol. 3, p. 187 .

WALKER, R. G.

1966. Infrared photometry of stars and planets. Ph. D. Thesis, Harvard Univ.

WILDT, R.

1934. Ozon und sauerstoff in den planeten-atmosphären.

Veroffentlichungen Univ. - Sternwarte Gottingen, No. 38.

WOOLF, N. J.

1965. Remarks made at the Conference on Remote Investigation of Martian Biology, Cambridge, Mass., January.

YOUNKIN, R. L.

1966. A search for limonite near-infrared spectral features on Mars. Astrophys. Journ., vol. 144, pp. 809-818. 


\section{BIOGRAPHICAL NOTES}

JAMES B. POLLACK received the A.B. degree from Princeton in 1960 , the M.A. degree from the University of California at Berkeley in 1962, and the $\mathrm{Ph} . \mathrm{D}$. degree from Harvard University in 1965.

Dr. Pollack currently holds an appointment as physicist on the staff of the Smithsonian Astrophysical Observatory. His research specialties include theoretical studies of planetary atmospheres, cloud layers, and surfaces.

CARL SAGAN received the A.B., S. B., S.M., and Ph.D。degrees from the University of Chicago in 1954, 1955, 1956, and 1960, respectively.

Since 1962 he has held joint appointments on the staffs of Harvard College Observatory and the Smithsonian Astrophysical Observatory. He is also a consultant to the National Aeronautics and Space Administration and the National Academy of Sciences.

Dr. Sagan's investigations include studies of the physics and chemistry of planetary atmospheres and surfaces, exobiology, and the origins of life. 